九州大学学術情報リポジトリ

Kyushu University Institutional Repository

\title{
前立腺癌放射線治療における照射間の非等方的直腸 形状変化の解析
}

モハンマド, ハェカル

ht tp://hdl. hand le. net/2324/1959083

出版情報：Kyushu University，2018，博士（保健学）， 課程博士 バージョン:

権利関係: $\odot 2018$ Associazione Italiana di Fisica Medica. Published by Elsevier Ltd. All rights reserved. 


\section{Doctoral Thesis}

Computational Analysis of Interfractional Anisotropic Shape Variations of the Rectum in Prostate Cancer Radiation Therapy

Division of Medical Quantum Science, Department of Health Sciences Graduate School of Medical Sciences, Kyushu University

Student ID: 3MD15510N

Mohammad Haekal

June 6, 2018 

Original paper

\title{
Computational analysis of interfractional anisotropic shape variations of the rectum in prostate cancer radiation therapy
}

\author{
Mohammad Haekal $^{\mathrm{a}}$, Hidetaka Arimura ${ }^{\mathrm{b}, *}$, Taka-aki Hirose ${ }^{\mathrm{a}}$, Yusuke Shibayama ${ }^{\mathrm{c}}$, Saiji Ohga ${ }^{\mathrm{b}}$, \\ Junichi Fukunaga ${ }^{\mathrm{c}}$, Yoshiyuki Umezuc ${ }^{\mathrm{c}}$, Hiroshi Honda ${ }^{\mathrm{b}}$, Tomonari Sasaki ${ }^{\mathrm{b}}$ \\ ${ }^{a}$ Graduate School of Medical Sciences, Kyushu University, 3-1-1, Maidashi, Higashi-ku, Fukuoka 812-8582, Japan \\ ${ }^{\mathrm{b}}$ Faculty of Medical Sciences, Kyushu University, 3-1-1, Maidashi, Higashi-ku, Fukuoka 812-8582, Japan \\ ${ }^{\mathrm{c}}$ Department of Medical Technology, Kyushu University Hospital, 3-1-1, Maidashi, Higashi-ku, Fukuoka 812-8582, Japan
}

\section{A R T I C L E I N F O}

\section{Keywords:}

Point distribution model

Prostate cancer radiation therapy

Rectum shape variation

Rectum overlap region

\begin{abstract}
A B S T R A C T
Purpose: To analyze the uncertainties of the rectum due to anisotropic shape variations by using a statistical point distribution model (PDM).

Materials and methods: The PDM was applied to the rectum contours that were delineated on planning computed tomography (CT) and cone-beam CT (CBCT) at 80 fractions of 11 patients. The standard deviations (SDs) of systematic and random errors of the shape variations of the whole rectum and the region in which the rectum overlapped with the PTV (ROP regions) were derived from the PDMs at all fractions of each patient. The systematic error was derived by using the PDMs of planning and average rectum surface determined from rectum surfaces at all fractions, while the random error was derived by using a PDM-based covariance matrix at all fractions of each patient.

Results: Regarding whole rectum, the population SDs were larger than $1.0 \mathrm{~mm}$ along all directions for random error, and along the anterior, superior, and inferior directions for systematic error. The deviation is largest along the superior and inferior directions for systematic and random errors, respectively. For ROP regions, the population SDs of systematic error were larger than $1.0 \mathrm{~mm}$ along the superior and inferior directions. The population SDs of random error for the ROP regions were larger than $1.0 \mathrm{~mm}$ except along the right and posterior directions.

Conclusions: The anisotropic shape variations of the rectum, especially in the ROP regions, should be considered when determining a planning risk volume (PRV) margins for the rectum associated with the acute toxicities.
\end{abstract}

\section{Introduction}

Prostate cancer was ranked as the fifth leading cause of death from cancer for men worldwide in 2012 [1]. Incidence rates are increasing every year in the developed countries such as United Kingdom and Japan [2]. Several options are available to treat the prostate cancer including radiation therapy which allowed the prostate to be treated with high dose of radiation while sparring surrounding normal tissues [3].

The quality of radiation therapy in prostate cancer treatment is affected by high dose regions which could be induced by patient movement, internal motion of the organ, and patient set-up errors $[4,5]$. Fig. 1 illustrates the anatomical regions of a rectum, bladder, and planning target volume (PTV) determined by radiation oncologists. Anterior parts of the rectum may overlap with the PTV due to large internal margins and/or rectal displacements as shown in Fig. 1. The rectal position uncertainties, which could cause toxicities (e.g., rectal bleeding, fecal incontinence), mainly comes from the rectal motion due to the changes in rectal filling [6-10]. The two common methods used to study the rectal motion were tracking the changes in rectal volume and evaluating the translation and rotation errors of the rectum [5,11-13]. Fontenla et al. [14], however, noted that the more complex problem of internal organ motion involve changes in the shape (shape variations) of the organ especially along the anterior direction of the rectum $[5,15]$. Therefore, the shape variations of the rectum, especially along the anterior direction, need to be investigated.

In order to dealt with the position uncertainties of the organs at risks (OARs), the International Commission on Radiation Units and Measurements (ICRU) reports no. 62 [16] and 83 [17] introduced the concept of planning risk volume (PRV) margins. In the case of prostate

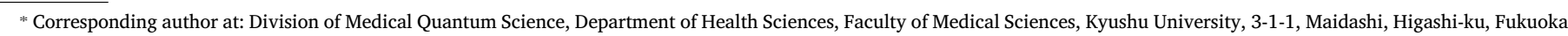
812-8582, Japan.

E-mail address: arimurah@med.kyushu-u.ac.jp (H. Arimura). 


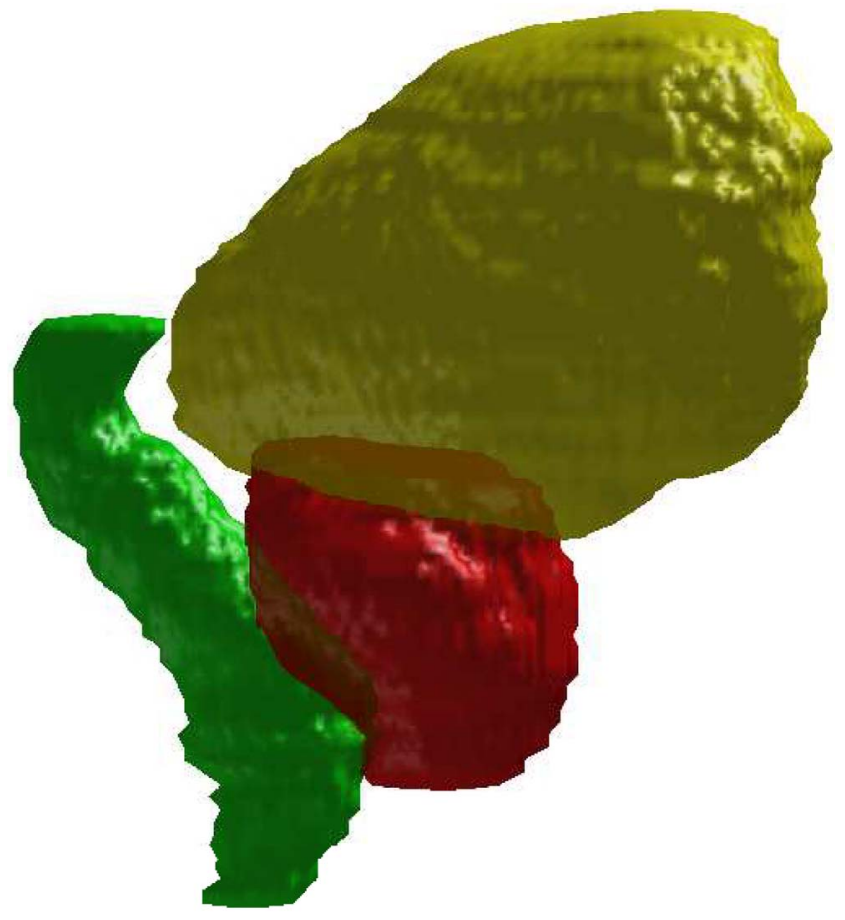

Fig. 1. An illustration of the anatomical regions of a rectum (green), bladder (yellow), and PTV (red) determined by radiation oncologists. Note that parts of the rectum and the bladder were overlapped with the PTV which could cause high-dose exposure to both OARs. (For interpretation of the references to colour in this figure legend, the reader is referred to the web version of this article.)

cancer radiation therapy, the use of PRV dose-volume histograms (DVHs) is recommended to predict acute rectal toxicity $[15,18,19]$. "Recipes" to determine the uniform PRV margins have been developed by McKenzie et al. and Stroom and Heijmen [20,21]. However, the uniform PRV margins are inadequate to represent the actual rectal variations during treatment, as noted by McKenzie et al. [20] and Prabhakar et al. [22]. Therefore, an application of anisotropic PRV margins of the rectum should be considered.

There have been three studies that dealt with the shape variations of the rectum. Hoogeman et al. [23] analyzed the quantification of local rectal wall displacements by calculating local systematic and random errors of the rectum along three directions where they unfolded the outer surface of the delineated rectal wall and projected the 3-space coordinates of each surface element to a 2D map. Sohn et al. [24] investigated the correlated motion of adjacent organ structures between prostate, bladder and rectum which were parametrized by using sets of corresponding surface points and calculated the displacements between surface points at each fraction. They did not calculate the systematic and random errors that could be used in determining anisotropic PRV margins. Brierley et al. [25] investigated the determination of the PTV based on the rectal shape variations by using finite element modeling. They did not investigate the geometric errors related to the determination of PRV margins.

None of the previously mentioned studies, including ICRU, investigated directly the shape variations of the rectum along each anatomical direction separately (anterior, posterior, superior, inferior, left and right). The investigation along separate anatomical directions is indispensable for determining the anisotropic PRV margins. There have been also no studies on the systematic and random errors of the region in which the rectum overlapped with the PTV along the anterior wall (ROP regions), even though the shape variations of the ROP regions may cause the regions to be included in high dose distributions which can lead to rectum toxicities. Therefore, this study aims to investigate the anisotropic shape variations of the rectum and the ROP regions for prostate cancer radiation therapy along separate anatomical direction (anterior, posterior, superior, inferior, left and right).

\section{Material and methods}

\subsection{Clinical study}

This study was performed with the approval of the Institutional Review Board of our university hospital. The clinical data used in this study were obtained from 11 patients (range: $60-75$ years; median age: 64 years; stage: T1-T3a, N0, M0), who had undergone intensity modulated radiation therapy (IMRT) for prostate cancer. The planning CT images were acquired from a CT scanner (Mx 8000, Philips, Amsterdam, Netherlands) with $512 \times 512$-pixel dimensions, $0.98 \mathrm{~mm}$ in-plane pixel size, and $2.0 \mathrm{~mm}$ slice thickness. Each patient received a dose of $76 \mathrm{~Gy}$ at 38 fractions using an accelerating voltage of $10 \mathrm{MV}$ on a linear accelerator (Varian Medical Systems Inc., Palo Alto, USA).

The analyses of systematic and random errors of interfractional anisotropic shape variations of the rectum were derived from contours delineated on the planning CT and cone-beam CT (СВCT) images at 80 fractions of 11 patients. CBCT scans were performed just before irradiation at 5-9 fractions (mean: 7.3) of each patient. The CBCT data were used for correcting target localization at each fraction and only acquired at the beginning of the week to reduce the dose received by the patients. A kilovoltage CBCT scanner (On-Board-Imager, Varian Medical Image Systems Inc., Palo Alto, USA) was used to perform the scans which produced images with $384 \times 384$ pixel dimensions, $1.17 \mathrm{~mm}$ in-plane pixel size, and $2.5 \mathrm{~mm}$ slice thickness. The delineations of the rectum contours were based on a consensus between a radiation oncologist (S.O.) and a medical physicist (T.H.) using a commercially available radiation treatment planning (RTP) system (Eclipse version 6.5 and 8.1; Varian Medical Systems Inc., Palo Alto, USA).

\subsection{Pre-processing}

The original planning CT and СBCT images were converted into isotropic images with an isovoxel size of $1.17 \mathrm{~mm}$ using a cubic interpolation method. The rectum structures delineated on both planning CT and CBCT images were also extracted and converted using a shapebased interpolation method [26].

In this study, we focused on the errors introduced by the interfractional organ motions of the rectum. To reduce the effect of intraobserver variation and delineation artifacts in the calculation [24], uniform-length rectums were used for all cases. The reference length was equal to the shortest length $(7 \mathrm{~cm})$ of a rectum between starting and ending slices (around anus to sigmoid positions) delineated on the CBCT images among all cases [27].

\subsection{Calculation of errors due to shape variations}

This study evaluated the local errors of the shape variations by dealing with them separately along each axis, as illustrated in Fig. 2. The $x, y$, and $z$ axes are each separated along their positive and negative directions corresponding to the anterior, posterior, superior, inferior, left and right directions. The local errors were calculated as the displacements of position vectors at points on 3D surfaces of the rectum (Fig. 3). The rotation errors of the rectum were included as shape variations since the errors also introduced the displacements of the surface points [28].

The flowchart to calculate errors due to shape variations from planning CT and CBCT images were described in Fig. 3. First, all rectums were registered using a centroid matching technique. Then, the surfaces of the registered rectums were triangulated using a marching cubes algorithm to obtain 3D surfaces of the rectum [29]. The produced surfaces consisted of many vertices, which were reduced to a similar number of vertices $(\sim 1000)$ using a quadric error metric method [30]. 


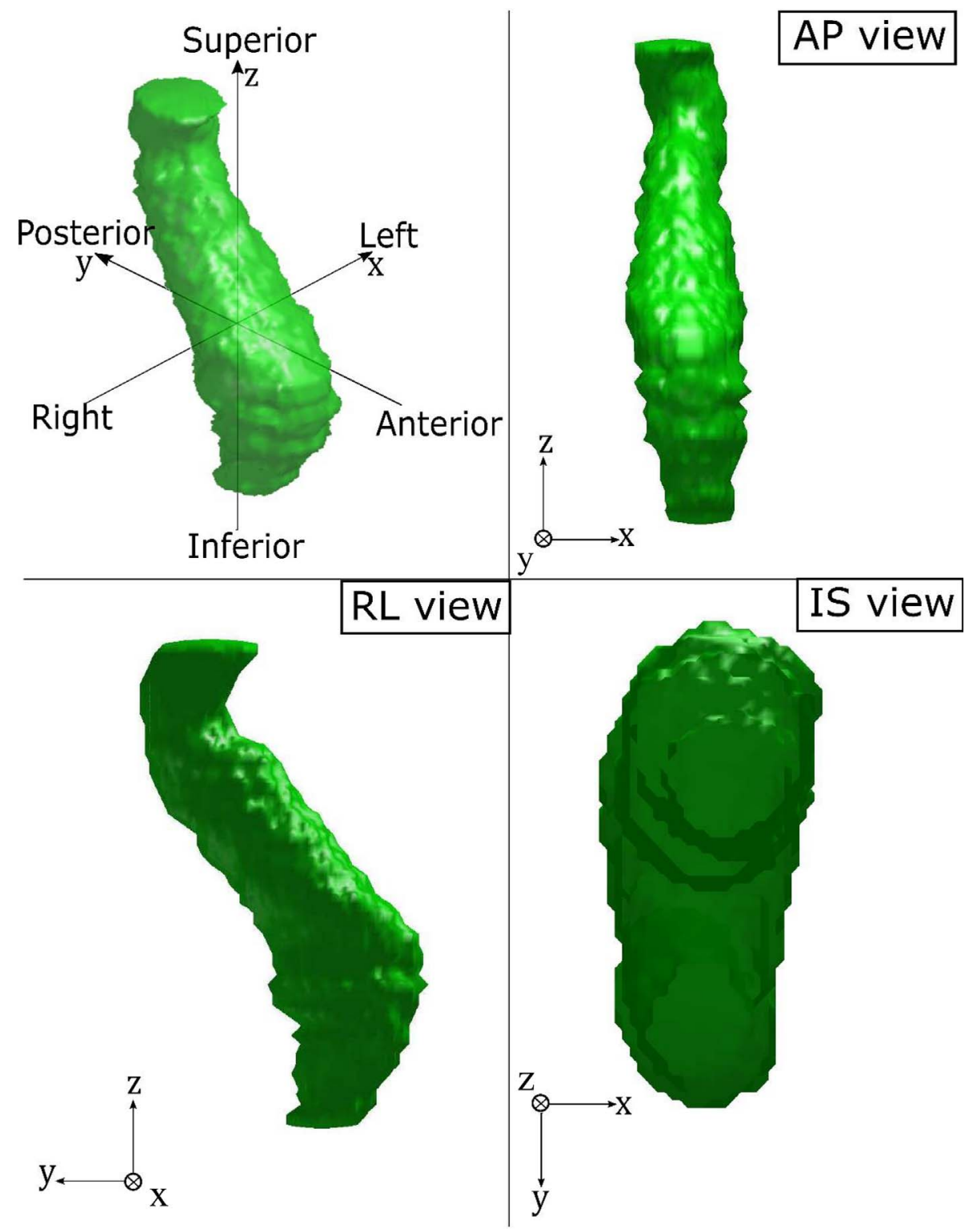

Fig. 2. Illustrations of anatomical directions describing rectum orientation. The " $x$ ", " $y$ " and " $z$ " axes correspond to the right-left (RL), anterior-posterior (AP) and inferior-superior (IS) directions, respectively.

Thin plate spline robust point matching (TPS-RPM) algorithm was applied to determine the correspondences between two surface structures. TPS-RPM algorithm is a non-rigid point matching algorithm that minimizes the distances between two point sets based on deterministic annealing and softassign algorithms. The general idea of the algorithm is to incorporate the thin-plate spline as a non-rigid mapping method to the robust point matching algorithm which is known to be only using affine and piecewise-affine mappings [31]. Let $X$ be a set of points from a source surface consisting of $\left\{x_{a}\right\}$ where ( $a=1,2, \ldots, H ; H$ : number of points) which correspondences with $V$, a set of points from a target surface consisting of $\left\{v_{b}\right\}$ where ( $b=1,2, \ldots, D ; D$ : number of points), are to be determined. The mapping of a point $x_{a}$ to a new location by a non-rigid transformation is represented by a general function $f$. The goal of the TPS-RPM is to minimize the following cost function based on the mapping function $f\left(x_{a}\right)$ between corresponding sets $\left\{x_{a}\right\}$ and $\left\{v_{b}\right\}$ :

$\min _{Z, f} \sum_{b=1}^{D} \sum_{a=1}^{H} z_{a b}\left\|v_{b}-f\left(x_{a}\right)\right\|^{2}+\lambda\|f\|^{2}$,

where $Z$ is the correspondence matrix consisting of $\left\{z_{a b}\right\}$, $\|f\|$ is the $\mathrm{L} 2$ norm, $\lambda$ is the hyper-parameter that trades off between the registration and regularization of $f$, and $\|f\|^{2}$ is the TPS regularizer which calculates the bending cost of $f$ and acts as a smoothness measure [31,32].

The geometrical errors of the rectum were classified into systematic and random errors [33,34]. The systematic and random errors for a group of similar patients can be described by using the standard deviations (SDs) $[17,33]$. In this study, the systematic error of a patient due to the shape variations was defined as the average deviation of a reference rectum from the planning rectum surface. The term "planning" rectum surface refers to the rectum surfaces derived from rectum structures on planning CT, while "reference" rectum surface refers to the average rectum surface determined from rectum surfaces at all fractions. The algorithm to construct a reference surface was described in a paper by Shibayama et al. [28]. On the other hand, the random error of a patient was defined as the SDs of the daily deviations of the rectum surface at each fraction from the reference rectum surface [17,35-37].

\subsubsection{Systematic error}

Fig. 4(a) illustrates the point distribution image of all the vertices on the reference rectum. To obtain the SDs of the systematic and random errors due to shape variations along each direction, the rectum was split into two halves (anterior and posterior regions, left and right regions, 


\begin{tabular}{c|} 
Rectum registration \\
using centroid matching
\end{tabular}

Surface triangulation

and vertices reduction
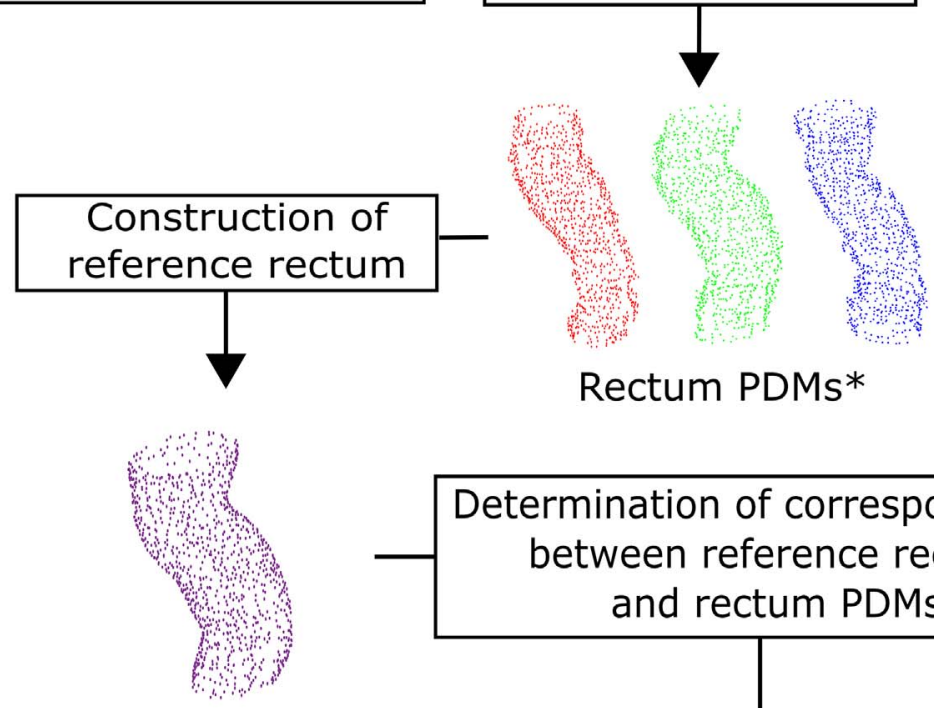

\section{Rectum PDMs*}

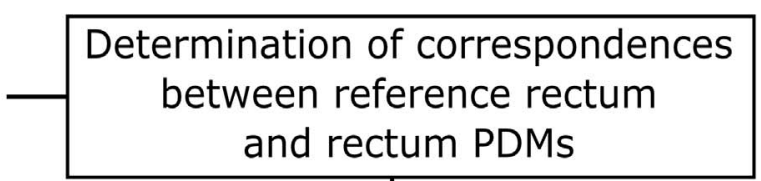

Reference rectum
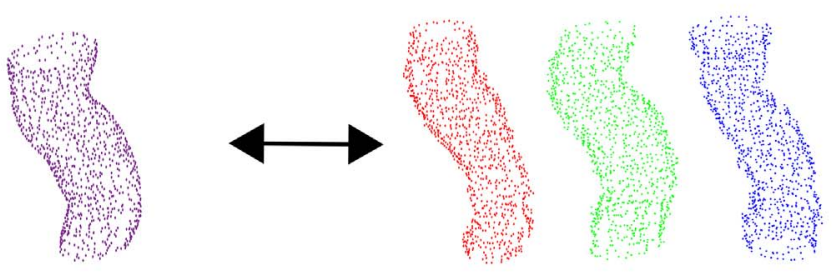

Reference rectum

\section{Rectum PDMs}

Fig. 3. A flowchart to calculate systematic and random errors of the rectum due to shape variations using a statistical point distribution models (PDMs).

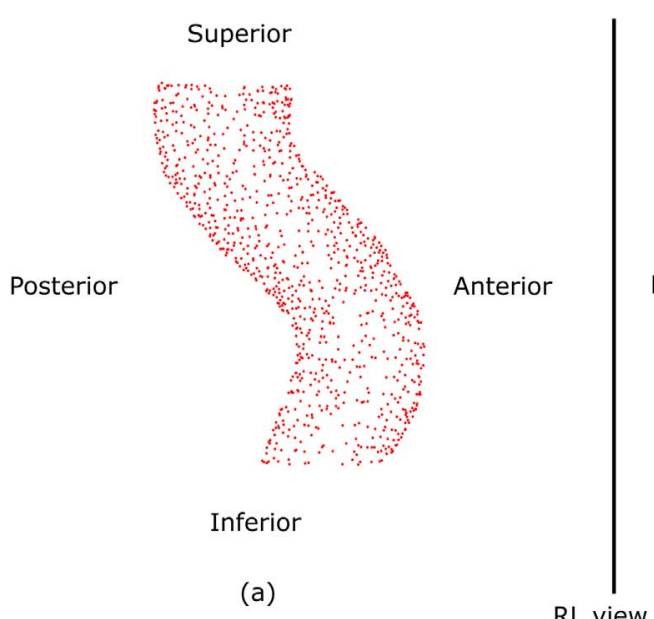

RL view
Superior

Posterior

for shape variations

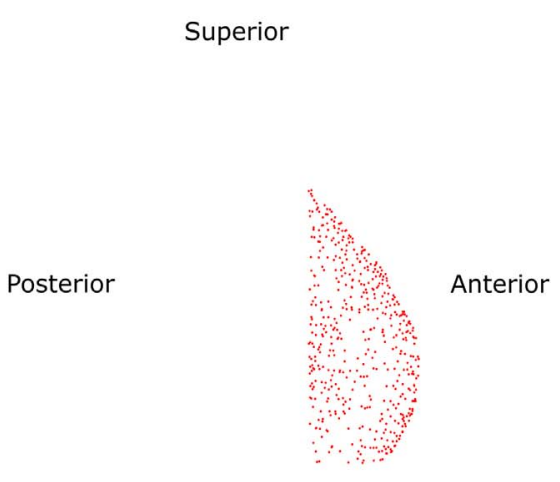

Inferior

(b)

Fig. 4. Point distributions images consisting of reference rectum vertices which are used to evaluate shape variations viewed from RL direction: (a) the PDM of whole vertices while, (b) only the anterior part of the rectum vertices split at the centroid remains to be used in evaluating the shape variations along the anterior direction. 


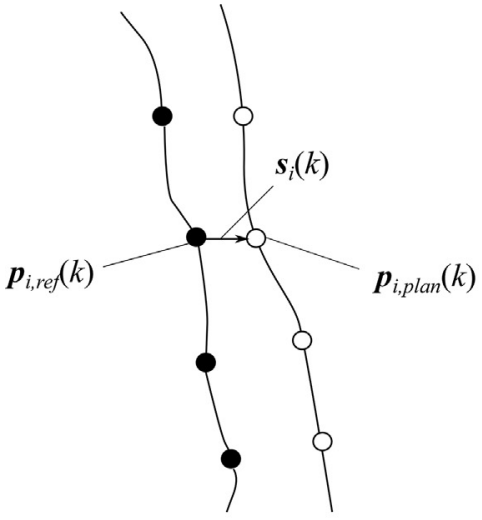

(a)

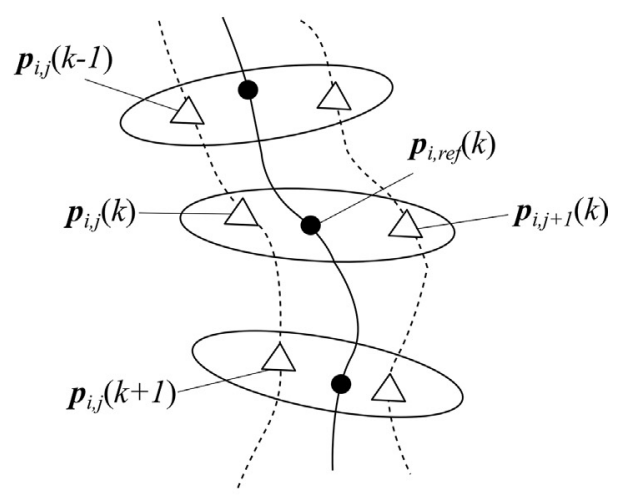

Fig. 5. Illustrations of the determinations of local systematic error and local random error due to shape variations: (a) local systematic error was calculated between reference rectum $(\bullet)$ and planning rectum $(O)$, while (b) local random error was calculated between reference rectum and rectum at each fraction $(\triangle)$. superior and inferior regions) with respect to its centroid, depending on which direction was evaluated. The example is shown in Fig. 4(b), where the anterior half vertices were used to calculate the SDs of the systematic and random errors along the anterior direction.

Fig. 5 illustrates the determinations of the local systematic and random errors due to the shape variations. Let $\boldsymbol{R}_{i, r e f}$ be the reference rectum of the $i$-th patient ( $i=1,2, \ldots, N ; N$ :number of patients) which corresponded with the rectum at each fraction. Accordingly, the vector $\boldsymbol{p}_{i, j}(k)$ of the $k$-th vertex $(k=1,2, \ldots, M ; M$ :number of vertices $)$ of the rectum at the $j$-th fraction $(j=1,2, \ldots, F ; F$ :number of fractions) of the $i$-th patient is corresponded to the vector of the $k$-th vertex $\left(\boldsymbol{p}_{i, \text { ref }}(k)=\left(p_{i, r e f, x}(k), p_{i, r e f, y}(k), p_{i, \text { ref }, z}(k)\right)^{\mathrm{T}}\right)$ at the reference rectum.

The correspondence between the 3D vector at the $k$-th vertex $\left(\boldsymbol{p}_{i, \text { plan }}(k)=\left(p_{i, \text { plan }, x}(k), p_{i, \text { plan }, y}(k), p_{i, \text { plan }, z}(k)\right)^{\mathrm{T}}\right)$ on the planning rectum $\boldsymbol{R}_{i, p l a n}$ and the vector of the $k$-th vertex $\boldsymbol{p}_{i, r e f}(k)$ on the reference rectum $\boldsymbol{R}_{i, r e f}$ was determined in a similar way. The local systematic error $\boldsymbol{s}_{i}(k)$ can be calculated as

$\boldsymbol{s}_{i}(k)=\boldsymbol{p}_{i, r e f}(k)-\boldsymbol{p}_{i, p l a n}(k)=\left(s_{i, x}(k), s_{i, y}(k), s_{i . z}(k)\right)^{\mathrm{T}}$,

where $s_{i, x}(k), s_{i, y}(k)$ and $s_{i . z}(k)$ represent the $x, y$ and $z$ components of the local systematic error vector in the inquired half, respectively. The average deviation $\varepsilon_{\mathrm{SV}, i, A}$ for a patient along the anterior direction, in this study, can be calculated as [21,37-39]

$\varepsilon_{\mathrm{SV}, i, A}=\frac{1}{M_{A}} \sum_{k=1}^{M_{A}} s_{i, y}(k)$.

Here, $M_{A}$ denotes the number of vertices in the anterior half of the rectum surfaces. Then, the population SD for all patients along the anterior direction can be obtained by [33,37-39]

$\varepsilon_{\mathrm{SV}, A}=\sqrt{\frac{1}{N} \sum_{i=1}^{N}\left(\varepsilon_{\mathrm{SV}, i, A}-\bar{\varepsilon}_{\mathrm{SV}, A}\right)^{2}}$,

where

$\bar{\varepsilon}_{\mathrm{SV}, A}=\frac{1}{N} \sum_{i=1}^{N} \varepsilon_{\mathrm{SV}, i, A}$.

The population SD of the systematic error along the other directions were also calculated in a same way. The SDs of the local systematic error $\tau_{i}(k)$ at each vertex were calculated as

$\tau_{i}(k)=\sqrt{s_{i, x}^{2}(k)+s_{i, y}^{2}(k)+s_{i, z}^{2}(k)}$.

\subsubsection{Random error}

The SDs of the interfractional random error due to shape variations were calculated by using a PDM-based covariance matrix. Let a point distribution column vector $\boldsymbol{q}_{i, j}$ of rectum $\boldsymbol{R}_{i, j}$ given by the elements of $\boldsymbol{p}_{i, j}(k)$ be

$\boldsymbol{q}_{i, j}=\left(p_{i, j, x}(1), \ldots, p_{i, j, x}(M), p_{i, j, y}(1), \ldots, p_{i, j, y}(M), p_{i, j, z}(1), \ldots, p_{i, j, z}(M)\right)^{\mathrm{T}}$.

The covariance matrix at all fractions of the $i$-th patient can be obtained by calculating

$\boldsymbol{V}_{i}=\frac{1}{F} \sum_{j=1}^{F}\left(\boldsymbol{q}_{i, j}-\overline{\boldsymbol{q}}_{i}\right)\left(\boldsymbol{q}_{i, j}-\overline{\boldsymbol{q}}_{i}\right)^{\mathrm{T}}$,

where

$\overline{\boldsymbol{q}}_{i}=\frac{1}{F} \sum_{j=1}^{F} \boldsymbol{q}_{i, j}$.

Here, the term $\overline{\boldsymbol{q}}_{i}$ denotes the mean vector of $\boldsymbol{q}_{i, j}$. The covariance matrix $\boldsymbol{V}_{i}$ consisted of diagonal elements which represent the square of SDs (variance) of the local random error at each vertex of the rectum. These elements can be defined as

$v_{\mathrm{SV}, i}$

$=\left(\sigma_{\mathrm{SV}, i, x}^{2}(1), \ldots, \sigma_{\mathrm{SV}, i, x}^{2}(M), \sigma_{\mathrm{SV}, i, y}^{2}(1), \ldots, \sigma_{\mathrm{SV}, i, y}^{2}(M), \sigma_{\mathrm{SV}, i, z}^{2}(1), \ldots, \sigma_{\mathrm{SV}, i, z}^{2}(M)\right)^{\mathrm{T}}$.

The variances on all vertices of the rectum were used to calculate the individual SD of the random error along each direction. The individual SD of a patient along the anterior direction $\left(\sigma_{\mathrm{SV}, i, A}\right)$ can be calculated as

$\sigma_{\mathrm{SV}, i, A}=\sqrt{\frac{1}{M_{A}} \sum_{k=1}^{M_{A}} \sigma_{\mathrm{SV}, i, y}^{2}(k)}$,

where $\sigma_{\mathrm{SV}, i, y}(k)$ denotes the local SD of the random error in the anterior half of the rectum and $M_{A}$ represents the number of vertices in the anterior half. The population SD of random error for all patients along the anterior direction $\left(\sigma_{\mathrm{SV}, A}\right)$ is defined as the root-mean-square (RMS) of the individual SDs of each patient in the group and calculated by $[33,38,39]$

$\sigma_{\mathrm{SV}, A}=\sqrt{\frac{1}{N} \sum_{i=1}^{N} \sigma_{\mathrm{SV}, i, A}^{2}}$.

The population SDs along the other directions (left, right, posterior, superior, and inferior) can be obtained using the same method. The SDs of the local random error on the $k$-th vertex $\left(\sigma_{\mathrm{SV}, i}(k)\right)$ were calculated as

$\sigma_{\mathrm{SV}, i}(k)=\sqrt{\sigma_{\mathrm{SV}, i, x}^{2}(k)+\sigma_{\mathrm{SV}, i, y}^{2}(k)+\sigma_{\mathrm{SV}, i, z}^{2}(k)}$. 


\section{Determination of correspondence between rectum at each fraction and reference rectum}

\section{Determination of reference PTV}

Fig. 6. A flowchart to calculate systematic and random errors of rectum-overlapped-with-PTV (ROP) regions due to shape variations using a statistical point distribution models (PDMs).

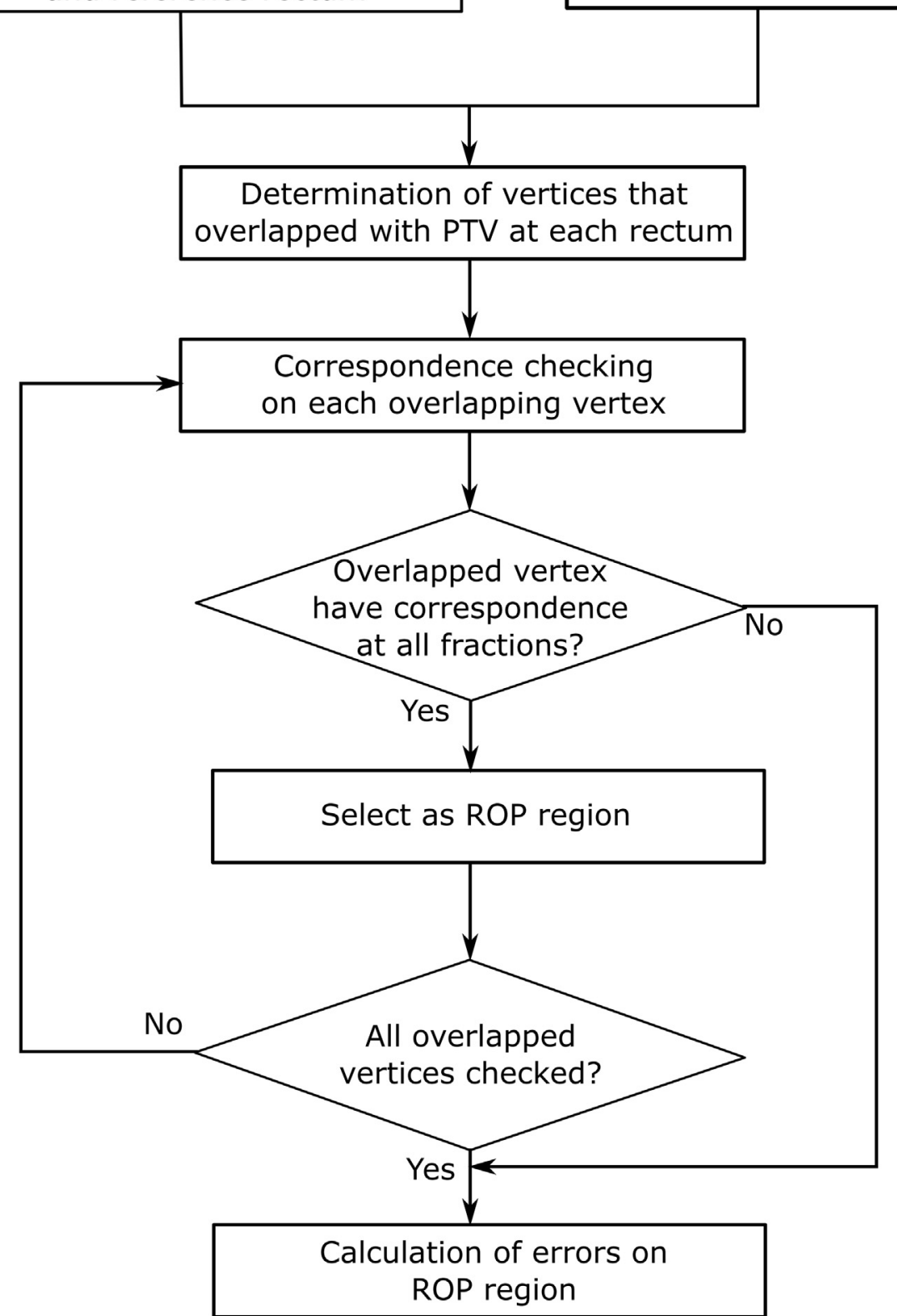

\subsection{Calculation of errors at ROP regions}

The regions in which the rectum overlapped with the PTV along the anterior wall (referred to as ROP regions) may be included in high-dose distributions which could lead to rectum toxicities such as rectal bleeding [19]. Therefore, the shape variations of the ROP regions were analyzed to improve the determination of the anisotropic PRV margins. The vertices of the rectum which were detected to be inside the PTV were selected. Then, the calculation of errors was conducted using similar principles described in Section 2.3.

\subsubsection{Determination of $R O P$ regions}

The overall procedure for determining the ROP regions of the rectum is described in Fig. 6. The determination of correspondence between rectum at each fraction and a reference rectum will produce the corresponding rectum $\widehat{\boldsymbol{R}}_{i, j}$, where $j$ represents the fraction number
( $j=1,2, \ldots, F ; F$ :number of fractions) of the $i$-th patient ( $i=1,2, \ldots, N ; N$ :number of patients). A reference PTV $\boldsymbol{T}_{i}$ of each patient was constructed by selecting the overlapped pixels between each PTV binary images at all fractions.

The overlapped vertices of the rectum $\hat{\boldsymbol{R}}_{\mathrm{OV}, i, j}$ at the $j$-th fraction of the $i$-th patient were identified by finding the vertices whose coordinates were identical with those of the reference PTV $\boldsymbol{T}_{i}$. The process can be intuitively described as

$\widehat{\boldsymbol{R}}_{\mathrm{OV}, i, j}=\widehat{\boldsymbol{R}}_{i, j} \cap \boldsymbol{T}_{i}$,

Then, an ROP region at each fraction of a patient can be obtained by selecting overlapped vertices that have correspondences at all fractions as illustrated in Fig. 7. 


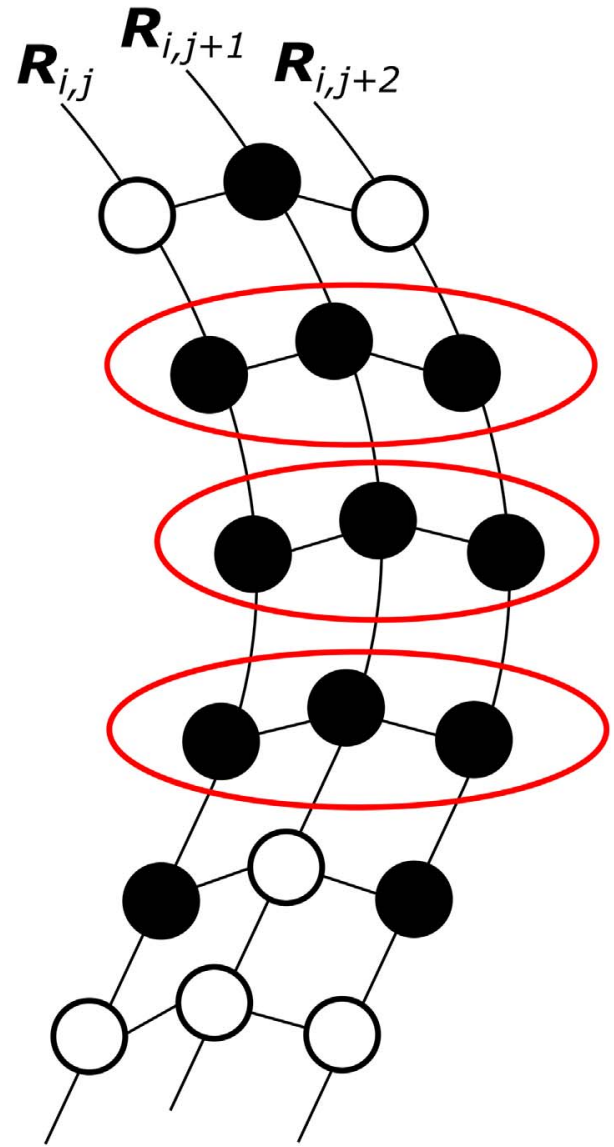

Fig. 7. An illustration of the determination of the ROP regions of the rectum. An overlapped vertex ( $\bullet$ would be included as ROP region (red border) if the correspondences with other overlapped vertices at all fractions were found. (For interpretation of the references to colour in this figure legend, the reader is referred to the web version of this article.)

\subsection{Dose evaluation study}

In order to demonstrate the impacts of the proposed method on prostate cancer treatment, the treatment plans with an anisotropic PRV margin were designed as simulations on the CBCT images to calculate the dose evaluation indices, CTV $D_{98}$ and $V_{75}, V_{70}, V_{65}, V_{60}$, and $V_{40}$ of the rectum. $D_{98}$ was defined as the percentage of dose received by $98 \%$ of $\mathrm{CTV}$, while $V_{x}$ was defined as the rectum volume receiving $x$ Gy. These dose evaluation indices were selected since $V_{70}$ and $V_{75}$ for the rectum were associated with Grade 2 or higher complications [40], while in another reports $V_{65}$ for the rectum was also associated with late toxicity [41-43] and $V_{40}-V_{60}$ for the rectum were found to be more relevant to the occurrence of severe late rectal bleeding $[44,45]$. Four new cases were employed as test cases for validation of the PRV margin determined based the shape variations, which were obtained by the proposed method in this study. The anisotropic PRV margin was derived along each direction by using the McKenzie's margin recipe of $1.3 \varepsilon+0.5 \sigma$ to the rectum [20]. Here, $\varepsilon$ and $\sigma$ denote the population SDs of the systematic (Eq. (4)) and random errors (Eq. (12)) of the rectum, respectively, which were calculated by the proposed method. The test cases were treated with IMRT with a total number of 21 fractions. A treatment plan based on the PRV margin was generated for each patient by applying plan constraints to the rectum PRV using a commercially available RTP system (Eclipse version 10.0; Varian Medical Systems Inc., Palo Alto, USA). The constraints were $V_{70}<5 \%, V_{65}<10 \%$, $V_{60}<20 \%$, and $V_{40}<40 \%$, which are based on QUANTEC recommendations to have been used in clinical practice $[44,46]$. A Wilcoxon signed-rank test was employed to compute the statistical difference between dose evaluation indices in original and PRV-based plans.

\section{Results}

Fig. 8 shows the population SDs of the systematic and random errors of the rectum due to shape variations along each anatomical direction of all patients. The population SDs for systematic errors were $0.6 \mathrm{~mm}$ along the left direction, $0.3 \mathrm{~mm}$ along the right direction, $1.0 \mathrm{~mm}$ along the anterior direction, $0.7 \mathrm{~mm}$ along the posterior direction, $2.1 \mathrm{~mm}$ along the inferior direction and $2.4 \mathrm{~mm}$ along the superior direction. The population SDs for random errors were $1.2 \mathrm{~mm}$ along the left direction, $1.2 \mathrm{~mm}$ along the right direction, $1.6 \mathrm{~mm}$ along the anterior direction, $1.6 \mathrm{~mm}$ along the posterior direction, $1.9 \mathrm{~mm}$ along the inferior direction and $1.7 \mathrm{~mm}$ along the superior direction.

Fig. 9 shows the comparisons of the population SDs for shape variations between the whole rectum and the ROP regions along each direction. For systematic errors of the ROP regions, the population SDs were smaller along all directions except along the left and right directions compared with the population SDs of the whole rectum. For random errors of the ROP regions, only the population SDs along the left direction was not changed, while along the other directions the population SDs were smaller compared with population SDs of the whole rectum. The population SDs was shown along the posterior direction indicating that the ROP regions were not limited to the anterior side of the rectum.

The population SDs of systematic errors for the ROP regions were $0.8 \mathrm{~mm}$ along the left direction, $0.8 \mathrm{~mm}$ along the right direction, $0.9 \mathrm{~mm}$ along the anterior direction, $1.9 \mathrm{~mm}$ along the inferior direction and $1.3 \mathrm{~mm}$ along the superior direction. The population SDs of random errors for ROP regions were $1.1 \mathrm{~mm}$ along the left direction, $0.8 \mathrm{~mm}$ along the right direction, $1.1 \mathrm{~mm}$ along the anterior direction, $0.1 \mathrm{~mm}$ along the posterior direction, $1.8 \mathrm{~mm}$ along the inferior direction and $1.6 \mathrm{~mm}$ along the superior direction.

In summary, the population SDs of the random errors for the whole rectum along all directions were larger than $1.0 \mathrm{~mm}$. On the other hand, the population SDs of the systematic errors for the whole rectum were smaller than $1.0 \mathrm{~mm}$ along the posterior, left, and right directions. The deviations along the superior direction was largest for the systematic errors, while the deviation along the inferior direction was largest for the random errors. The population SDs of systematic errors for the ROP regions were larger than $1.0 \mathrm{~mm}$ along the superior and inferior directions, while the population SDs of random errors for the ROP regions were larger than $1.0 \mathrm{~mm}$ except along the right and posterior directions.

Fig. 10 shows the comparison of average dose evaluation indices between original and PRV-based plans for the 4 test patients. The $V_{75}$ for the rectum in PRV-based plans was significantly smaller $(\mathrm{p}<.001)$ than those of the original plans. However, the $V_{40}$ for the rectum in PRVbased plans was significantly larger $(\mathrm{p}<.001)$ than those of the original plans. On the other hand, the $D_{98}$ to the CTV showed no statistical significant difference between original and PRV-based plans $(\mathrm{p}>.001)$.

\section{Discussion}

The deviation along the superior, inferior, and anterior directions were dominant for systematic and random errors. Brierley et al. and Nuyttens et al. [25,47] noted similar observations of large deviations along superior and inferior directions of the rectum. The deviations were affected largely by the variabilities of other organ proximal to the rectum such as small bowel [47].

Figs. 11 and 12 illustrate the SDs of the local systematic and random errors visualized on the reference rectum for a patient. The calculations of the magnitudes of the local systematic errors and local random errors were performed by using Eqs. (6) and (13), respectively. Large variations $(>3.0 \mathrm{~mm}$ ) occurred in the upper-left and the upper-anterior 

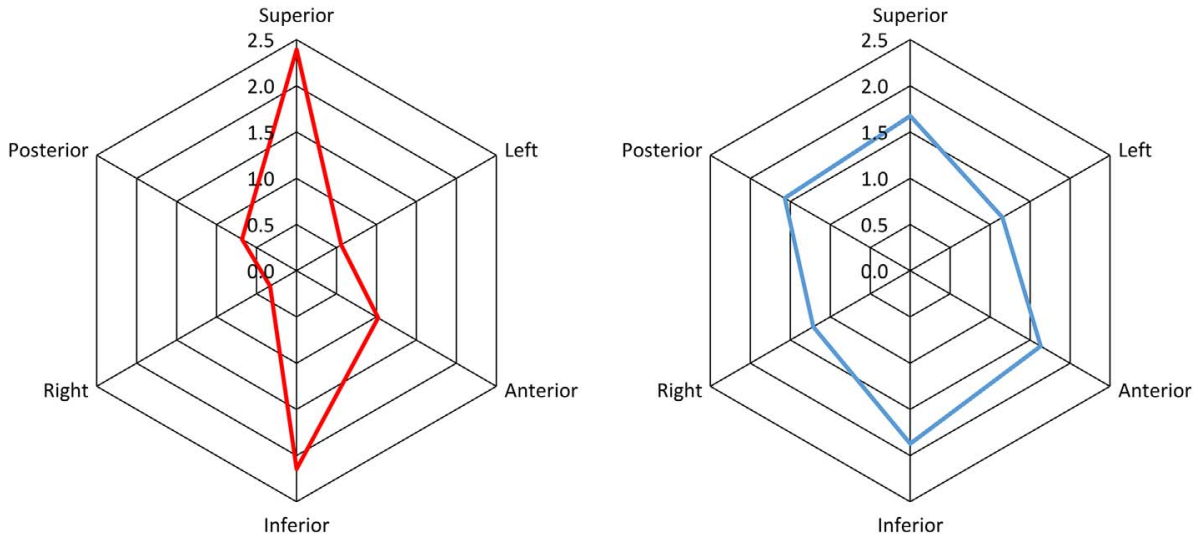

(a)

Systematic error

(b)

Random error
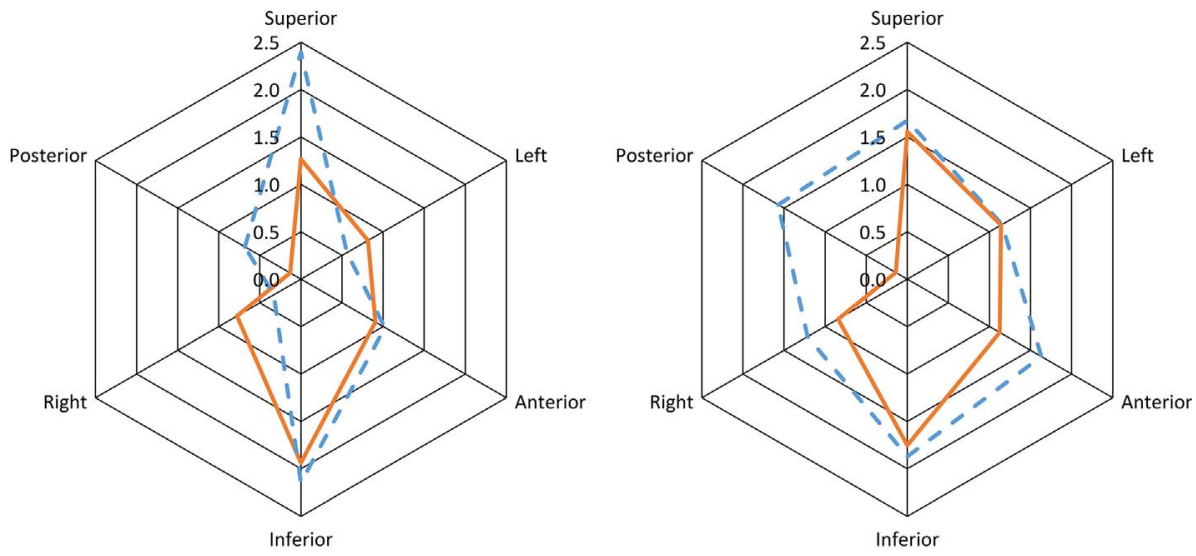

Whole rectum

(a)

ROP region

(b)
Fig. 8. The population SDs in mm of (a) systematic errors and (b) random errors of all patients due to shape variations along each anatomical direction. sides of the rectum for systematic errors. For random errors, beside the upper-left and upper-anterior sides, the lower-left and lower-anterior side of the rectum also had variations larger than $3.0 \mathrm{~mm}$. Hoogeman et al. [23] reported that their study also resulted in the largest variations occurred in the upper-anterior side of the rectum and uniformly distributed in the lower half of the rectum for random errors. The smaller variations in the posterior side of the rectum were indicating the possibilities of the sacrum limiting rectal wall movement. The various SDs distributed on the rectum surface, as visualized in Figs. 11 and 12 , showed that our method had more advantage in analyzing rectum shape variations than volume-based methods since our method considered every voxel on the surface in the calculation.

Fig. 13(a) and (b) illustrate the SDs of the local systematic and random errors of ROP region visualized on the reference rectum for a

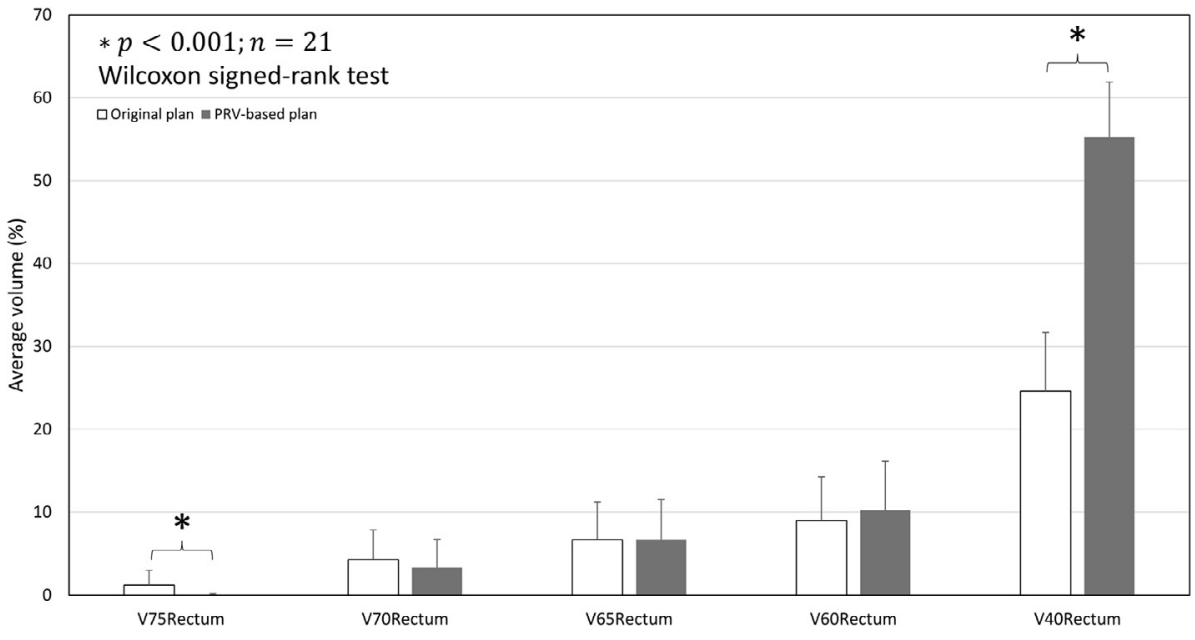

Fig. 10. Comparisons of average dose evaluation indices of the rectum (rectum $V_{75}, V_{70}, V_{65}, V_{60}$, and $V_{40}$ ) between original and PRV-based plans. 


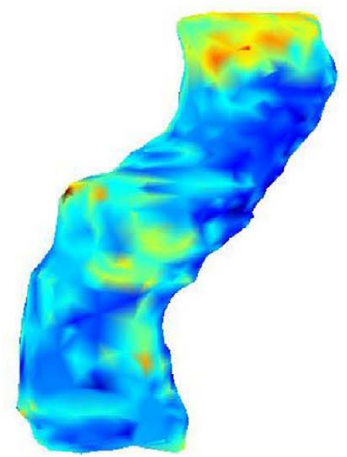

(a)

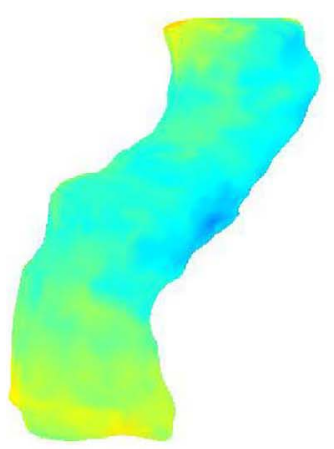

(a)

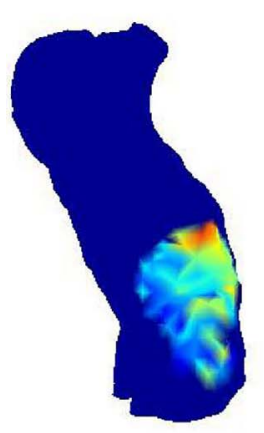

(a)

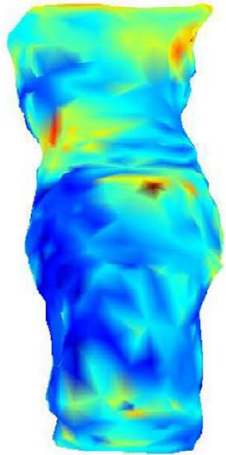

(b)

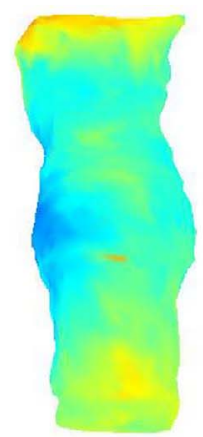

(b)

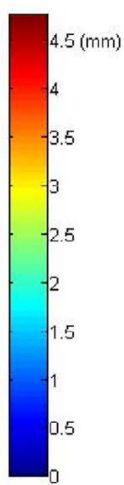

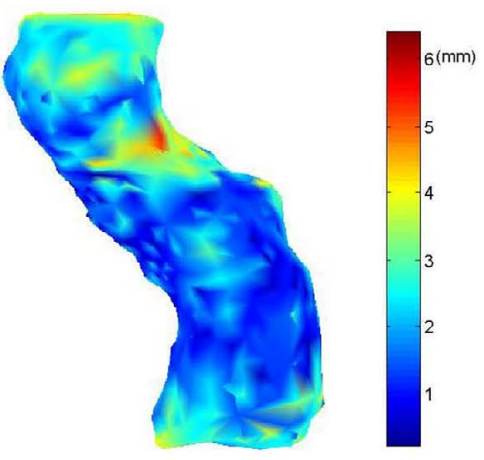

(c)
Fig. 11. Illustrations of the local systematic error SDs visualized on the reference rectum of a patient: (a) LR view, (b) AP view and (c) RL view of rectum.
Fig. 12. Illustrations of the local random error SDs visualized on the reference rectum of a patient: (a) LR view, (b) AP view and (c) RL view of rectum.
Fig. 13. Illustrations of the SDs of the local systematic and random error of an ROP region visualized on the reference rectum of a patient: (a) local systematic and (b) local random error. patient. Large variation $(>3.0 \mathrm{~mm}$ ) for systematic errors occurred in the upper part of the ROP regions. On the other hand, the variations $(>2.0 \mathrm{~mm}$ ) were uniformly distributed in the ROP regions for random errors. Aside from rectal gas and peristalsis activity [28], the lower part of the rectum was also suggested to be affected from the contraction and relaxation of the muscle system of the pelvic floor [23]. Variation larger than $3.0 \mathrm{~mm}$ in the ROP regions indicated that more portions of the rectum were exposed by the high dose region, consequently increasing the chance of acute toxicity in the rectum [19].

Figs. 14 and 15 show the relationships between the SDs of dose evaluation indices and the individual SDs of systematic and random errors of rectum shape variations along the anterior direction, respectively. These results indicate that rectum shape variations affected variations of dose evaluation indices, but there were no statistical significances between them along all directions ( $\mathrm{p}>.05$, Pearson correlation test).

The high dose to the ROP regions and large regions receiving low doses need to be studied in details to reduce rectum late toxicities and late gastrointestinal (GI) quality of life (QOL) in IMRT for prostate cancer in future studies. The ROP region is defined as the region in which the rectum overlapped with the PTV along the anterior wall and may be included in high-dose distribution. Chennupati et al. [42] reported that a high dose to the anterior rectal wall was associated with a low scoring of late GI QOL. On the other hand, as for large regions receiving low doses, dose constraints such as $V_{40}<65-80 \%$ were recommended to reduce the incidence rate of fecal incontinence to $1-2 \%$ [48]. Since $V_{40}$ obtained by the proposed method was smaller than $60 \%$ , the risk of fecal incontinence may be negligible for the cases used in this study.

The smaller population SDs of the ROP regions than for the whole rectum may be used to improve the anisotropic PRV margins used in prostate cancer radiation treatment. A large margin for PRV of parallel organs such as the rectum could become problem if the PRV is used for dose evaluation and criteria determination [21]. Since the rectal motion was reported to be occurring mainly along the anterior direction $[5,15]$, the solution for providing a more compact margin can be obtained by deriving the PRV margins from the errors of the ROP regions especially along the anterior direction. By deriving the PRV margins based on the 

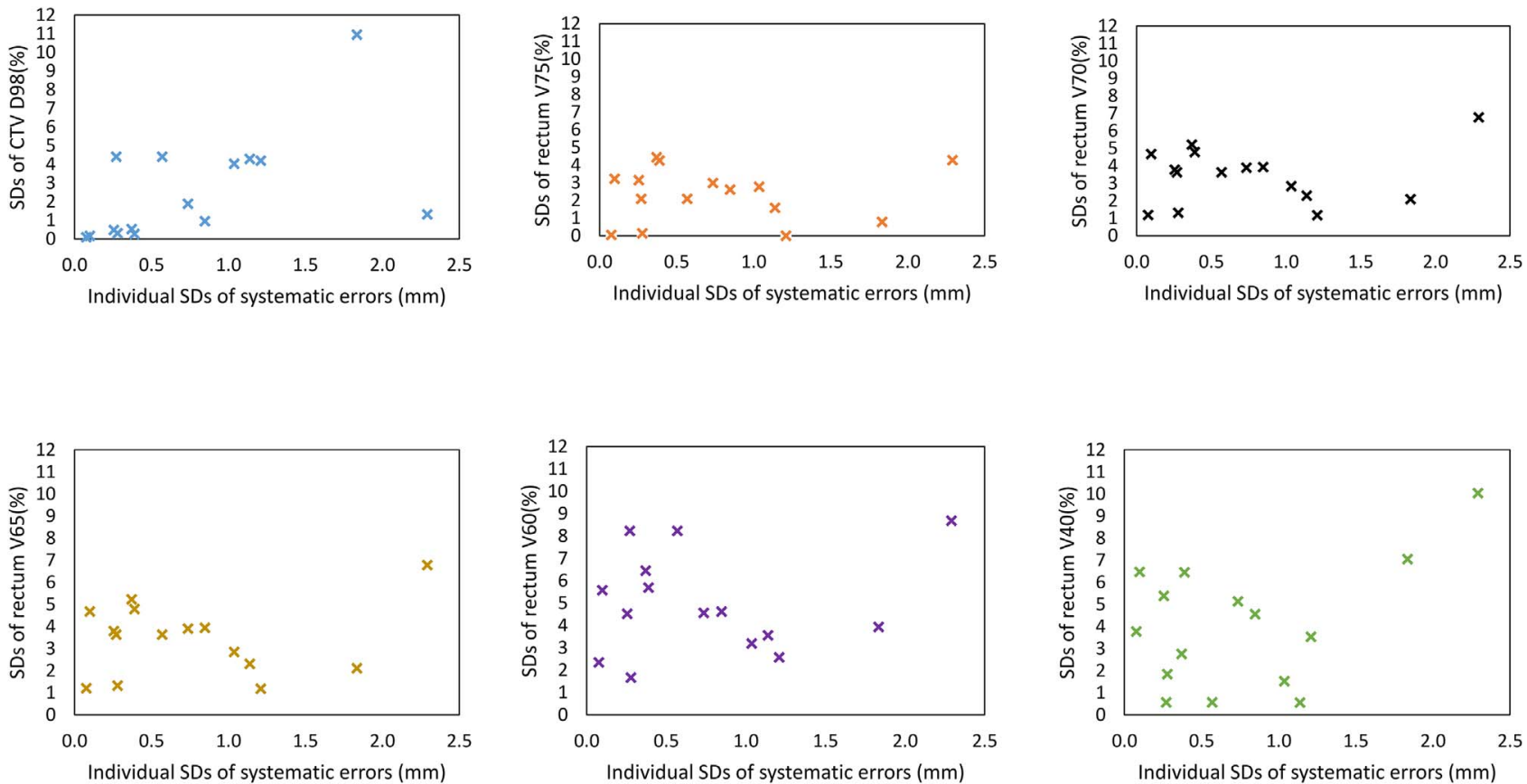

Fig. 14. Relationships between the SDs of dose evaluation indices and the individual SDs of systematic errors of rectum shape variations for 15 cases along the anterior direction.
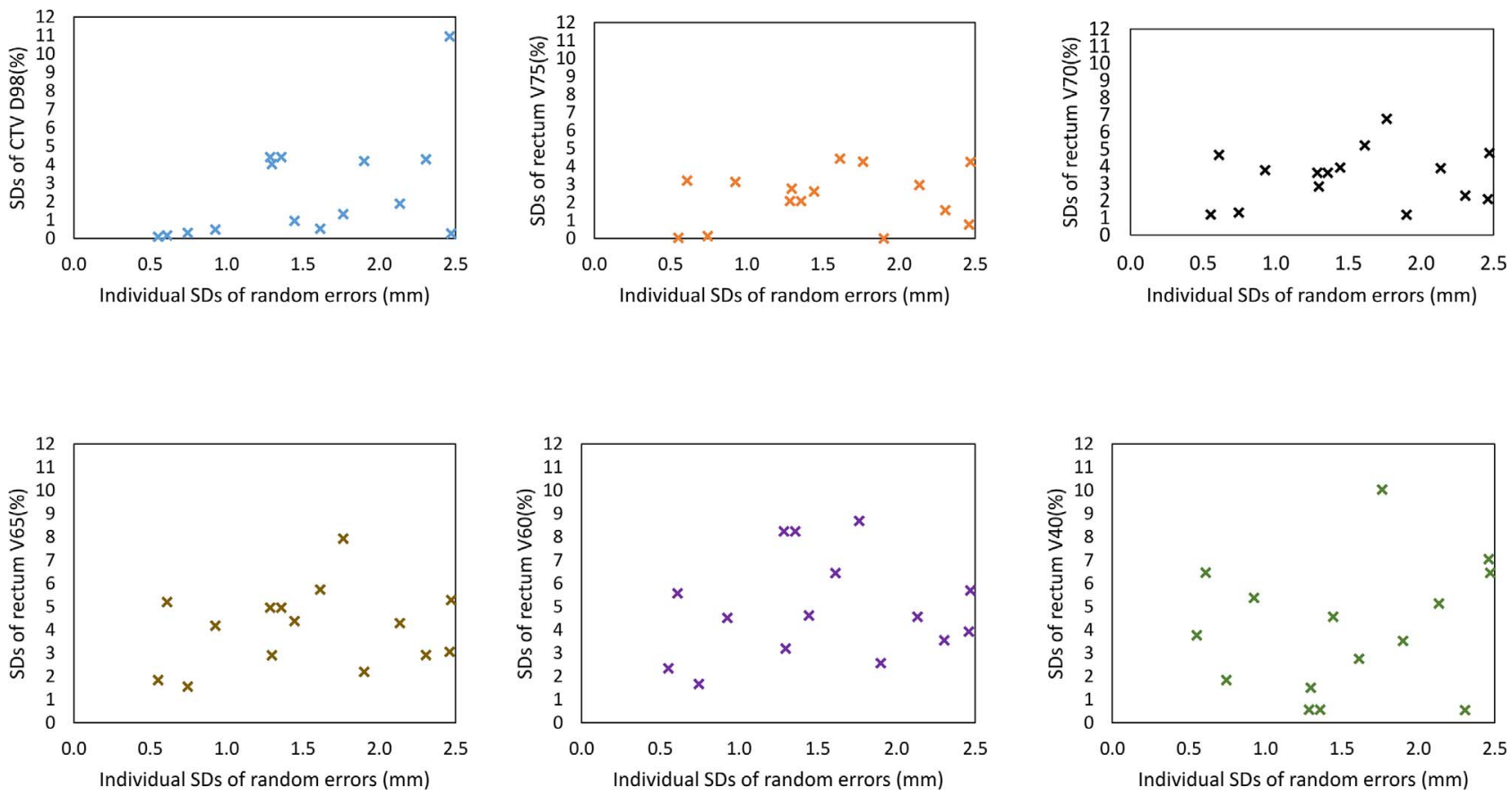

Fig. 15. Relationships between the SDs of dose evaluation indices and the individual SDs of random errors of rectum shape variations for 15 cases along the anterior direction.

errors of the ROP regions, a smaller margin can be produced without neglecting the variations of the rectum wall proximal to the high-dose regions. Smaller margins have also been indicated to be useful in predicting late morbidity of the rectum [49].

There are some limitations in this study. A larger number of cases is necessary to improve the statistical estimation of shape variations of the rectum. Since the TPS-RPM does not consider preserving the geometrical features of the surface, a more advanced correspondence determination method such as free-form deformation (FFD) used in selforganizing deformable model (SDM) would also enhance the precision of the calculation of errors [50,51]. Nevertheless, this study will be useful as a means to study the shape variations of the rectum. The results are envisioned as a means to derive anisotropic PRV margins for the rectum which can be utilized for general RTP systems.

\section{Conclusions}

An analysis of interfractional anisotropic rectum shape variations using a statistical PDM in a computational framework has been presented. The population SDs for the whole rectum calculated by the proposed method were larger than $1.0 \mathrm{~mm}$ along all directions for random errors, while for systematic errors the population SDs were smaller than $1.0 \mathrm{~mm}$ along the posterior, left, and right directions. The population SDs of systematic errors for ROP regions calculated by the 
proposed method were larger than $1.0 \mathrm{~mm}$ along the superior and inferior directions. The population SDs of random errors calculated by the proposed method for the ROP regions were larger than $1.0 \mathrm{~mm}$ except along the right and posterior directions. The results suggest that the anisotropic shape variations analysis, especially in the ROP regions, should be considered when determining a PRV margins for the rectum associated with the acute toxicities.

\section{Conflict of interest statement}

None.

\section{Acknowledgements}

The authors would like to send the utmost gratitude to all members in Arimura laboratory, which had contribute a great deal of efforts in the performance of this study.

\section{Appendix A. Supplementary data}

Supplementary data associated with this article can be found, in the online version, at http://dx.doi.org/10.1016/j.ejmp.2017.12.019.

\section{References}

[1] Chen R, Ren S, Yiu MK, Fai NC, Cheng WS, Ian LH, et al. Prostate cancer in Asia: a collaborative report. Asian J Urol 2014;1:15-29. http://dx.doi.org/10.1016/j.ajur. 2014.08.007.

[2] Torre LA, Bray F, Siegel RL, Ferlay J, Lortet-Tieulent J, Jemal A. Global cancer statistics, 2012. CA Cancer J Clin 2015;65:87-108. http://dx.doi.org/10.3322/caac. 21262 [doi].

[3] Denmeade SR, Isaacs JT. A history of prostate cancer treatment. Nat Rev Cancer 2002;2:389-96. http://dx.doi.org/10.1038/nrc801.

[4] Langen KM, Jones DTL. Organ motion and its management. Int J Radiat Oncol Biol Phys 2001;50:265-78. http://dx.doi.org/10.1016/S0360-3016(01)01453-5.

[5] Muren LP, Ekerold R, Kvinnsland Y, Karlsdottir À, Dahl O. On the use of margins for geometrical uncertainties around the rectum in radiotherapy planning. Radiother Oncol 2004;70:11-9. http://dx.doi.org/10.1016/j.radonc.2003.11.013.

[6] Lebesque JV, Bruce AM, Guus Kroes AP, Touw A, Shouman T, van Herk M. Variation in volumes, dose-volume histograms, and estimated normal tissue complication probabilities of rectum and bladder during conformal radiotherapy of T3 prostate cancer. Int J Radiat Oncol Biol Phys 1995;33:1109-19. http://dx.doi.org/10.1016/ 0360-3016(95)00253-7.

[7] Roeske JC, Forman JD, Mesina CF, He T, Pelizzari CA, Fontenla E, et al. Evaluation of changes in the size and location of the prostate, seminal vesicles, bladder, and rectum during a course of external beam radiation therapy. Int J Radiat Oncol Biol Phys 1995;33:1321-9. http://dx.doi.org/10.1016/0360-3016(95)00225-1.

[8] Zelefsky MJ, Fuks Z, Happersett L, Lee HJ, Ling CC, Burman CM, et al. Clinical experience with intensity modulated radiation therapy (IMRT) in prostate cancer. Radiother Oncol 2000;55:241-9. http://dx.doi.org/10.1016/S0167-8140(99) 00100-0.

[9] Zelefsky MJ, Fuks Z, Hunt M, Yamada Y, Marion C, Ling CC, et al. High-dose intensity modulated radiation therapy for prostate cancer: early toxicity and biochemical outcome in 772 patients. Int J Radiat Oncol Biol Phys 2002;53:1111-6. http://dx.doi.org/10.1016/S0360-3016(02)02857-2.

[10] Foppiano F, Fiorino C, Frezza G, Greco C, Valdagni R. The impact of contouring uncertainty on rectal 3D dose-volume data: results of a dummy run in a multicenter trial (AIROPROS01-02). Int J Radiat Oncol Biol Phys 2003;57:573-9. http://dx.doi. org/10.1016/S0360-3016(03)00659-X.

[11] Zelefsky MJ, Crean D, Mageras GS, Lyass O, Happersett L, Clifton Ling C, et al. Quantification and predictors of prostate position variability in 50 patients evaluated with multiple CT scans during conformal radiotherapy. Radiother Oncol 1999;50:225-34. http://dx.doi.org/10.1016/S0167-8140(99)00011-0.

[12] van Herk M, Bruce A, Guus Kroes AP, Shouman T, Touw A, Lebesque JV. Quantification of organ motion during conformal radiotherapy of the prostate by three dimensional image registration. Int J Radiat Oncol Biol Phys 1995;33:1311-20. http://dx.doi.org/10.1016/0360-3016(95)00116-6.

[13] Rijkhorst EJ, Lakeman A, Nijkamp J, de Bois J, van Herk M, Lebesque JV, et al. Strategies for online organ motion correction for intensity-modulated radiotherapy of prostate cancer: prostate, rectum, and bladder dose effects. Int J Radiat Oncol Biol Phys 2009;75:1254-60. http://dx.doi.org/10.1016/j.ijrobp.2009.04.034.

[14] Fontenla E, Pelizzari CA, Roeske JC, Chen GT. Using serial imaging data to model variabilities in organ position and shape during radiotherapy. Phys Med Biol 2001;46:2317-36. http://dx.doi.org/10.1088/0031-9155/46/9/304.

[15] Dias RS, Giordani AJ, Souhami L, Segreto RA, Segreto HRC. Rectal planning risk volume correlation with acute and late toxicity in 3-dimensional conformal radiation therapy for prostate cancer. Technol Cancer Res Treat 2011;10:585-90.

[16] International Commission on Radiation Units and Measurements. ICRU Report 62.
Prescribing, Recording, and Reporting Photon Beam Therapy (Supplement to ICRU Report 50). J ICRU 1999: Ix + 52. doi: 10.1259/bjr.74.879.740294.

[17] ICRU. Prescribing, Recording and Reporting Photon-Beam Intensity Modulated Radiation Therapy (IMRT) (ICRU Report 83). vol. 10. 2010. doi: 10.1093/jicru/ ndq025.

[18] Muren LP, Karlsdottir Á, Kvinnsland Y, Wentzel-Larsen T, Dahl O. Testing the new ICRU 62 "planning organ at risk volume" concept for the rectum. Radiother Oncol 2005;75:293-302. http://dx.doi.org/10.1016/j.radonc.2005.03.007.

[19] Prabhakar R, Oates R, Daryl J, Chang J, Geso M, Cramb J. Rectal complication probability from composite volumes derived from daily cone beam computed tomography in prostate cancer radiotherapy. J Cancer Res Ther 2016;12:374-8. http://dx.doi.org/10.4103/0973-1482.174529.

[20] McKenzie A, Van Herk M, Mijnheer B. Margins for geometric uncertainty around organs at risk in radiotherapy. Radiother Oncol 2002;62:299-307. http://dx.doi. org/10.1016/S0167-8140(02)00015-4.

[21] Stroom JC, Heijmen BJM. Limitations of the planning organ at risk volume (PRV) concept. Int J Radiat Oncol 2006;66:279-86. http://dx.doi.org/10.1016/j.ijrobp. 2006.05.009.

[22] Prabhakar R, Oates R, Jones D, Kron T, Cramb J, Foroudi F, et al. A study on planning organ at risk volume for the rectum using cone beam computed tomography in the treatment of prostate cancer. Med Dosim 2014;39:38-43. http://dx. doi.org/10.1016/j.meddos.2013.09.003.

[23] Hoogeman MS, Van Herk M, De Bois J, Muller-Timmermans P, Koper PCM, Lebesque JV. Quantification of local rectal wall displacements by virtual rectum unfolding. Radiother Oncol 2004;70:21-30. http://dx.doi.org/10.1016/j.radonc. 2003.11.015.

[24] Sohn M, Birkner M, Yan D, Alber M. Modelling individual geometric variation based on dominant eigenmodes of organ deformation: implementation and evaluation. Phys Med Biol 2005;50:5893-908. http://dx.doi.org/10.1088/0031-9155/50/24/ 009.

[25] Brierley JD, Dawson LA, Sampson E, Bayley A, Scott S, Moseley JL, et al. Rectal motion in patients receiving preoperative radiotherapy for carcinoma of the rectum. Int J Radiat Oncol Biol Phys 2011;80:97-102. doi: S0360-3016(10)00142-2 [pii] 10.1016/j.ijrobp.2010.01.042

[26] Herman GT, Zheng J, Bucholtz CA. Shape-based interpolation. IEEE Comput Graph Appl 1992;12:69-79. http://dx.doi.org/10.1109/38.135915.

[27] Hoogeman MS, van Herk M, Yan D, Boersma LJ, Koper PCM, Lebesque JV. A mode to simulate day-to-day variations in rectum shape. Int $\mathrm{J}$ Radiat Oncol Biol Phys 2002;54:615-25. http://dx.doi.org/10.1016/S0360-3016(02)02946-2.

[28] Shibayama Y, Arimura H, Hirose T, Nakamoto T, Sasaki T, Ohga S, et al. Investigation of interfractional shape variations based on statistical point distribution model for prostate cancer radiation therapy. Med Phys 2017;44:1837-45. http://dx.doi.org/10.1002/mp.12217.

[29] Lorensen WE, Cline HE. Marching cubes: a high resolution 3D surface construction algorithm. Comput Graph (ACM) 1987;21:163-9. http://dx.doi.org/10.1145/ 37402.37422 .

[30] Garland M, Heckbert PS. Surface simplification using quadric error metrics. Proc. 24th Annu. Conf. Comput. Graph. Interact. Tech. - SIGGRAPH '97, 1997, p. 209-16. doi: $10.1145 / 258734.258849$.

[31] Chui H, Rangarajan A. A new point matching algorithm for non-rigid registration. Comput Vis Image Underst 2003;89:114-41. http://dx.doi.org/10.1016/S1077. 3142(03)00009-2.

[32] Huang SH, Pan J, Mulcaire G, Abbeel P. Leveraging appearance priors in non-rigid registration, with application to manipulation of deformable objects. IEEE Int. Conf. Intell. Robot. Syst. 2015;2015:878-85. http://dx.doi.org/10.1109/IROS.2015. 7353475 .

[33] Stroom JC, Heijmen BJM. Geometrical uncertainties, radiotherapy planning margins, and the ICRU-62 report. Radiother Oncol 2002;64:75-83. http://dx.doi.org/ 10.1016/S0167-8140(02)00140-8.

[34] Van Herk M. Errors and margins in radiotherapy. Semin Radiat Oncol 2004;14:52-64. http://dx.doi.org/10.1053/j.semradonc.2003.10.003.

[35] Wu J, Haycocks T, Alasti H, Ottewell G, Middlemiss N, Abdolell M, et al. Positioning errors and prostate motion during conformal prostate radiotherapy using on-line isocentre set-up verification and implanted prostate markers. Radiother Oncol 2001;61:127-33. http://dx.doi.org/10.1016/S0167-8140(01)00452-2.

[36] Witte MG, van der Geer J, Schneider C, Lebesque JV, van Herk M. The effects of target size and tissue density on the minimum margin required for random errors. Med Phys 2004;31:3068-79. http://dx.doi.org/10.1118/1.1809991.

[37] Arimura H, Shibayama Y, Haekal M, Jin Z, Ikushima K. Computer-assisted target volume determination. In: Arimura $\mathrm{H}$, editor. Image-Based Comput. Radiat. Ther. Singapore: Springer Singapore; 2017. p. 87-109. http://dx.doi.org/10.1007/978981-10-2945-5 5

[38] Strbac B, Jokic VS. Evaluation of set-up errors in head and neck radiotherapy using electronic portalimaging. Phys Medica 2013;29:531-6. http://dx.doi.org/10.1016/ j.ejmp.2012.12.001.

[39] Dzierma Y, Beyhs M, Palm J, Niewald M, Bell K, Nuesken F, et al. Set-up errors and planning margins in planar and СBCT image-guided radiotherapy using three different imaging systems: a clinical study for prostate and head-and-neck cancer. Phys Medica 2015;31:1055-9. http://dx.doi.org/10.1016/j.ejmp.2015.09.002.

[40] Huang EH, Pollack A, Levy L, Starkschall G, Dong L, Rosen I, et al. Late rectal toxicity: dose-volume effects of conformal radiotherapy for prostate cancer. Int $\mathrm{J}$ Radiat Oncol Biol Phys 2002;54:1314-21. http://dx.doi.org/10.1016/S03603016(02)03742-2.

[41] Pederson AW, Fricano J, Correa D, Pelizzari CA, Liauw SL. Late toxicity after intensity-modulated radiation therapy for localized prostate cancer: an exploration of dose-volume histogram parameters to limit genitourinary and gastrointestinal 
toxicity. Int J Radiat Oncol Biol Phys 2012;82:235-41. http://dx.doi.org/10.1016/ j.ijrobp.2010.09.058.

[42] Chennupati SK, Pelizzari CA, Kunnavakkam R, Liauw SL. Late toxicity and quality of life after definitive treatment of prostate cancer: redefining optimal rectal sparing constraints for intensity-modulated radiation therapy. Cancer Med 2014;3:954-61. http://dx.doi.org/10.1002/cam4.261.

[43] Someya M, Hori M, Tateoka K, Nakata K, Takagi M, Saito M, et al. Results and DVH analysis of late rectal bleeding in patients treated with 3D-CRT or IMRT for localized prostate cancer. J Radiat Res 2014;56:122-7. http://dx.doi.org/10.1093/jrr/ rru080.

[44] Michalski JM, Gay H, Jackson A, Tucker SL, Deasy JO. Radiation dose-volume effects in radiation-induced rectal injury. Int J Radiat Oncol Biol Phys 2010:76. http://dx.doi.org/10.1016/j.ijrobp.2009.03.078.

[45] Coates J, El Naqa I. Outcome modeling techniques for prostate cancer radiotherapy: data, models, and validation. Phys Medica 2016;32:512-20. http://dx.doi.org/10. 1016/j.ejmp.2016.02.014.

[46] Buschmann M, Seppenwoolde Y, Wiezorek T, Weibert K, Georg D. Advanced optimization methods for whole pelvic and local prostate external beam therapy. Phys Medica 2016;32:465-73. http://dx.doi.org/10.1016/j.ejmp.2016.03.002.
[47] Nuyttens JJ, Robertson JM, Yan D, Martinez A. The variability of the clinical target volume for rectal cancer due to internal organ motion during adjuvant treatment. Int J Radiat Oncol Biol Phys 2002;53:497-503. http://dx.doi.org/10.1016/S03603016(02)02753-0.

[48] Landoni V, Fiorino C, Cozzarini C, Sanguineti G, Valdagni R, Rancati T. Predicting toxicity in radiotherapy for prostate cancer. Phys Medica 2016;32:521-32. http:// dx.doi.org/10.1016/j.ejmp.2016.03.003.

[49] Thor M, Væth M, Karlsdottir A, Muren LP. Rectum motion and morbidity prediction: improving correlation between late morbidity and DVH parameters through use of rectum planning organ at risk volumes. Acta Oncol (Madr) 2010;49:1061-8. http://dx.doi.org/10.3109/0284186X.2010.505200.

[50] Morooka K, Nagahashi H. Self-organizing deformable model: A new method for fitting mesh model to given object surface. Lect. Notes Comput. Sci. (including Subser. Lect. Notes Artif. Intell. Lect. Notes Bioinformatics), vol. 3804 LNCS, 2005, p. 151-8. doi: 10.1007/11595755_19.

[51] Miyauchi S, Morooka K, Miyagi Y, Fukuda T, Tsuji T, Kurazume R. Tissue surface model mapping onto arbitrary target surface based on self-organizing deformable model. Emerg. Secur. Technol. (EST), 2013 Fourth Int. Conf. on., Cambridge: IEEE; 2013, p. 79-82. doi: 10.1109/EST.2013.34. 



\section{Computational Analysis of Interfractional Anisotropic Shape Variations of the Rectum in Prostate Cancer Radiation Therapy \\ Department of Health Sciences \\ 3MD15510N \\ Mohammad Haekal}

Purpose: To analyse the uncertainties of the rectum due to anisotropic shape variations by using a statistical point distribution model (PDM).

Materials and Methods: The PDM was applied to the rectum contours that were delineated on planning computed tomography (CT) and cone-beam CT (CBCT) at 80 fractions of 11 patients. The standard deviations (SDs) of systematic and random errors of the shape variations of the whole rectum and the region in which the rectum overlapped with the planning target volume (PTV), referred to as the ROP region, were derived from the PDMs at all fractions of each patient. The systematic error was derived by using the PDMs of the planning rectum surface and an average rectum surface which was determined from rectum surfaces at all fractions. Meanwhile, the random error was derived by using a PDM-based covariance matrix at all fractions of each patient.

Results: The population SDs of the whole rectum were larger than $1.0 \mathrm{~mm}$ along the anterior, superior and inferior directions for the systematic error and larger than $1.0 \mathrm{~mm}$ along all direction for the random error. For ROP regions, the population SDs were larger than $1.0 \mathrm{~mm}$ along the superior and inferior directions for the systematic error and larger than $1.0 \mathrm{~mm}$ along superior, inferior, anterior, and left directions for the random error.

Conclusions: The anisotropic shape variations of the rectum, especially in ROP regions, should be considered in the analysis of the rectum uncertainties in prostate cancer radiation therapy.

Keywords: prostate cancer radiation therapy; rectum shape variation; rectum overlap region; point distribution model 
タイトル: 前立腺癌放射線治療における照射間の非等方的直腸形状変化の解析

目的: 本研究では前立腺癌放射線治療における照射間の非等方的直腸形状変化を解析 した。

方法：11 症例の 80 分割照射における planning computed tomography (CT) と conebeam CT （CBCT）画像の輪郭情報に点分布モデルを適応した。点分布モデルを用いて、 直腸の全領域及び planning target volume（PTV)で重なっている領域（ROP 領域）に おいて、患者毎の分割照射間形状変動の系統誤差と偶然誤差の標準偏差 $(\mathrm{SD})$ を求め た。形状変動の系統誤差を治療計画の直腸表面と平均直腸表面(患者ごとの全分割照 射中に得られた直腸の平均形状) 間の平均偏差として計算した。形状変動の偶然誤差 は患者毎の全照射に得られた点分布の共分散行列から計算した。

結果 : 各方向における全患者の直腸の系統誤差と偶然誤差の SD は $1.0 \mathrm{~mm}$ 以上とな った。ROP 領域では、SI 方向の系統誤差の SD は $1.0 \mathrm{~mm}$ となり、各方向の偶然誤差 の $\mathrm{SD}$ は $1.0 \mathrm{~mm}$ 以上となった。

結論：前立腺癌の放射線治療計画において、直腸形状の不確実性を解析する際には、 直腸の非等方性的な形状変動、特に ROP 領域の非等方性的な変動を考慮する必要が ある。

キーワード: 放射線治療計画; 前立腺癌; 直腸形状変動; 点分布モデル 


\section{ACKNOWLEDGEMENTS}

الحمدالله ربّ العالمين, all praise belong to Allah, the Owner of All Knowledge, who grace me with an insignificant drop from His infinite sea of knowledge, by which I hope could be used to make the world a better place by Allah's will.

I would like to express gratitude to my supervisor, Prof. Hidetaka Arimura, for his time, patience, and invaluable advices during my time as his student which helped me shaped up as a researcher. I am also thankful for the example he has provided as a successful researcher.

I would like to thank all Department of Clinical Radiology staff members, especially Mr. Takaaki Hirose and Mr. Yusuke Shibayama whose contribution and ideas have become an essential part of this research. I would also like to thank all faculty members of Division of Medical Quantum Science, Department of Health Sciences, Faculty of Medical Sciences in Kyushu University and all Arimura Laboratory members who provided me with precious comments and advices.

I would also like to extend my infinite gratitude and respect for my parents, Dadang Kahmad and Cahya Komala, who cared for me since the day I was born and always believe in me. And also for my parents, Erie Sandhita and Indah Renaningsih, who always support me and include me in their $d u$ 'a, May Allah 'azza wa jalla bless them with infinite greatness both in this world and the hereafter. Also for my brothers (bang Roma, Zaky, a Yudi, mas Aldy, mas Wawan) and sisters (Nida, teh Huda, Nisa, mbak Ajeng, mbak Anis) and their children who always support me, thank you so much.

My very special gratitude is for the one who ease my heart in difficult times, laugh with me in happy times, whose smile washed away my weariness every time, jalan atthirari anni, Karine Ravienna. Never let go of my hands.

Finally, for all who have helped me survive this phase of adventure in life: G-Lounge, Fisika 2007, Keluarga Cemara, PPI Fukuoka, MusFuk, and many other kind-hearted souls that extend their hands and spirits to make this world worth living, I take off my hat to you all. 


\section{Table of Contents}

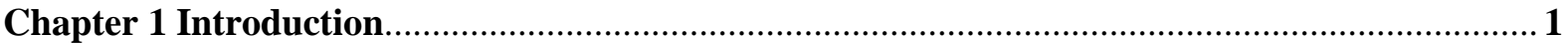

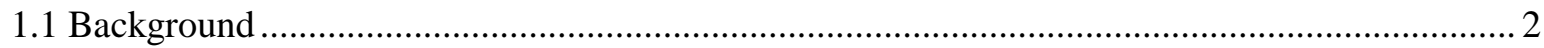

1.2 Purpose

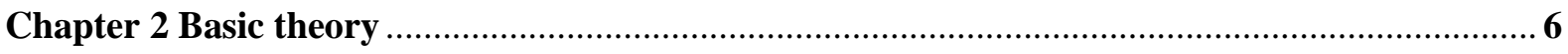

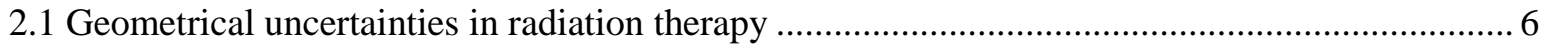

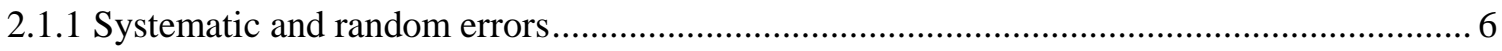

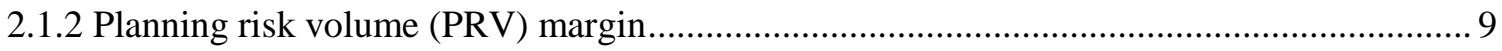

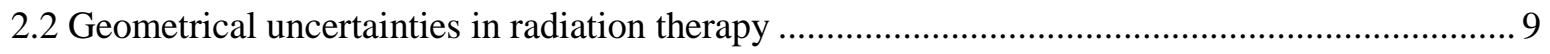

2.2.1 Surface triangulation by the marching cubes algorithm .................................................. 10

2.2.2 Surface simplification by the quadric error metrics method .............................................. 11

2.2.3 PDM correspondences determination by using the thin-plate spline robust point matching

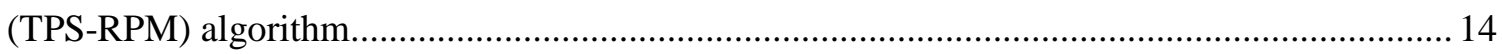

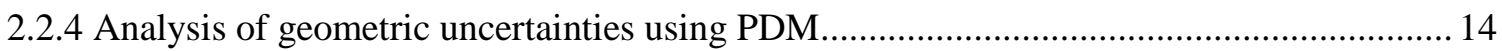

Chapter 3 Computational analysis of interfractional anisotropic shape variations of the rectum

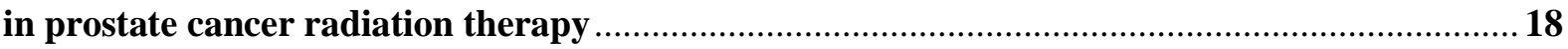

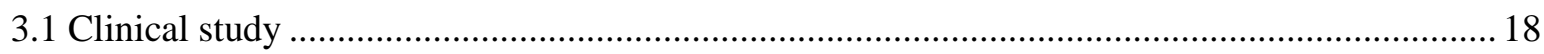

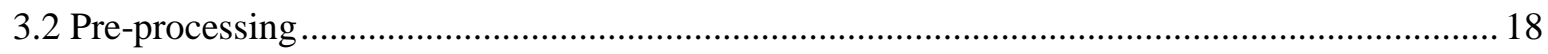

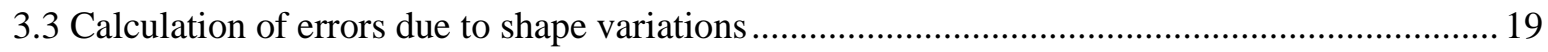

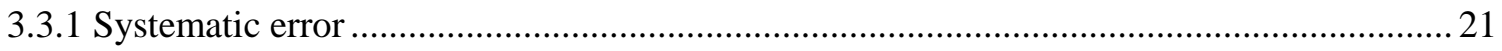

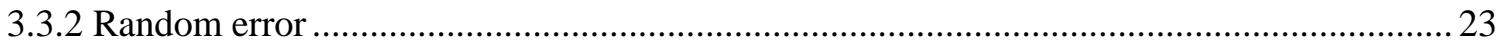

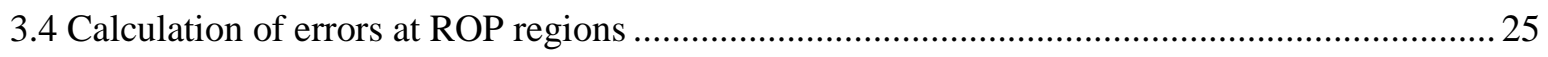

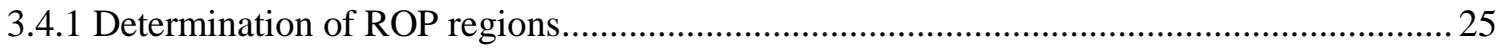


3.5 Dose evaluation study

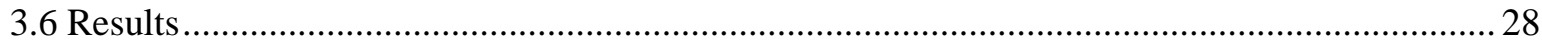

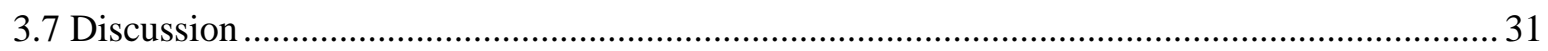

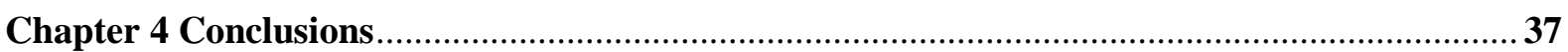

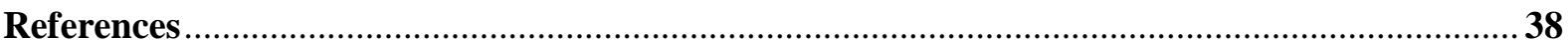

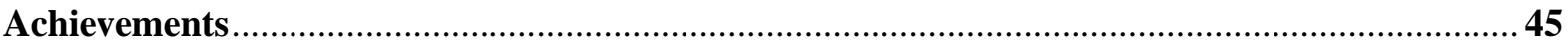




\section{List of Figures}

Figure 1.1 An illustration of treatment options for patient diagnosed with prostate cancer 1

Figure 1.2 A visualization of the interfractional shape variations of rectum (magenta contour) and bladder (cyan contour) with respect to the PTV (red contour), as delineated by radiation oncologists . 2 Figure 1.3 Visualizations of the ROP region of a patient at a fraction as determined by the radiation oncologists 3

Figure 1.4 An illustration of the application of PRV margin to rectum structure for prostate cancer radiation therapy

Figure 2.1 An illustration of the systematic and random errors of an organ for a population of $N$ patients

Figure 2.2 An illustration of vertex, edge, and face of a triangulated surface.

Figure 2.3 Illustrations of the triangulation patterns used in marching cubes algorithm

Figure 2.4 Illustrations of pair contraction in the quadric error metrics algorithm

Figure 2.5 An illustration of a point distribution model of an object

Figure 3.1 Illustrations of anatomical directions describing rectum orientation

Figure 3.2 A flowchart to calculate systematic and random errors of the rectum due to shape variations using a statistical point distribution models (PDMs) 20

Figure 3.3 Point distribution images consisting of reference rectum vertices which are used to evaluate shape variations viewed from RL direction

Figure 3.4 Illustrations of the determinations of local systematic error and local random error due to shape variations

Figure 3.5 A flowchart to calculate systematic and random errors of rectum-overlapped-with-PTV (ROP) regions due to shape variations using a statistical point distribution models (PDMs) 
Figure 3.7 The population SDs in mm of systematic errors and random errors of all patients due to shape variations along each anatomical direction.

Figure 3.8 The population SDs in mm of whole rectum and ROP regions of the rectum: systematic error and random errors of all patients due to shape variations along each direction

Figure 3.9 Comparisons of average dose evaluation indices of the rectum (rectum $V_{75}, V_{70}, V_{65}, V_{60}$ and $V_{40}$ ) between original and PRV-based plans.

Figure 3.10 Illustrations of the local systematic error SDs visualized on the reference rectum of a patient

Figure 3.11 Illustrations of the local random error SDs visualized on the reference rectum of a patient

Figure 3.12 Illustrations of the SDs of the local systematic and random errors of an ROP region visualized on the reference rectum of a patient

Figure 3.13 Relationships between the SDs of dose evaluation indices and the individual SDs of systematic errors of rectum shape variations for 15 cases along the anterior direction

Figure 3.14 Relationships between the SDs of dose evaluation indices and the individual SDs of random errors of rectum shape variations for 15 cases along the anterior direction 35 


\begin{tabular}{|c|c|c|}
\hline \multicolumn{3}{|c|}{ bbreviations and keywords } \\
\hline Abbreviation & Full spelling & Explanation \\
\hline CBCT & $\begin{array}{l}\text { Cone-beam Computed } \\
\text { Tomography }\end{array}$ & $\begin{array}{l}\text { A variant type of computed tomography (CT), which uses } \\
\text { cone-shaped x-ray beam and two dimensional detectors } \\
\text { instead of fan-shaped x-ray beam and one dimensional } \\
\text { detectors. }\end{array}$ \\
\hline DVH & Dose-volume Histogram & $\begin{array}{l}\text { A plot of a cumulative dose-volume frequency distribution } \\
\text { which graphically summarizes the simulated radiation } \\
\text { distribution within a volume of interest of a patient which } \\
\text { would result from a proposed radiation treatment plan. }\end{array}$ \\
\hline IMRT & $\begin{array}{l}\text { Intensity-modulated } \\
\text { Radiation Therapy }\end{array}$ & $\begin{array}{l}\text { An advanced form of three-dimensional conformal } \\
\text { radiotherapy which used non-uniform intensity of the } \\
\text { radiation beams and computerized inverse planning. }\end{array}$ \\
\hline OAR & Organs at Risk & $\begin{array}{l}\text { Non-target tissues that could suffer significant morbidity and } \\
\text { might influence the treatment planning and/or the absorbed } \\
\text { dose prescription if irradiated. }\end{array}$ \\
\hline PRV & Planning Risk Volume & $\begin{array}{l}\text { A geometrical concept that add margins to surround the OARs, } \\
\text { in order to consider the uncertainties and variations in the } \\
\text { position of the OARs to avoid serious complications. }\end{array}$ \\
\hline PTV & Planning Target Volume & $\begin{array}{l}\text { A geometrical concept introduced to ensure that the prescribed } \\
\text { dose will actually be delivered to all parts of the CTV with a } \\
\text { clinically acceptable probability, despite geometrical } \\
\text { uncertainties such as organ motion and setup variations, by } \\
\text { adding margins surrounding the CTV. }\end{array}$ \\
\hline ROP & Rectum-overlap PTV & $\begin{array}{l}\text { The region in which the rectum overlaps with the PTV in } \\
\text { prostate cancer radiation treatment. }\end{array}$ \\
\hline TPS-RPM & $\begin{array}{l}\text { Thin-plate Spline Robust } \\
\text { Point Matching }\end{array}$ & $\begin{array}{l}\text { A non-rigid point matching algorithm used for non-rigid } \\
\text { registration that utilizes the softassign, deterministic } \\
\text { annealing, the thin-plate spline for the spatial mapping and } \\
\text { outlier rejection to solve the correspondence and mapping } \\
\text { parameters. }\end{array}$ \\
\hline
\end{tabular}




\section{Chapter 1 Introduction}

\subsection{Background}

Prostate cancer was ranked as the fifth leading cause of death from cancer for men worldwide in 2012 (Chen et al. 2014). Incidence rates are increasing every year in the developed countries such as United Kingdom and Japan (Torre et al. 2015). Figure 1.1 shows several options to treat patient diagnosed with prostate cancer. Radiation therapy is one of the commonly used treatment approaches that allows us to treat the prostate with high dose of radiation while sparring surrounding normal tissues (Denmeade and Isaacs 2002).

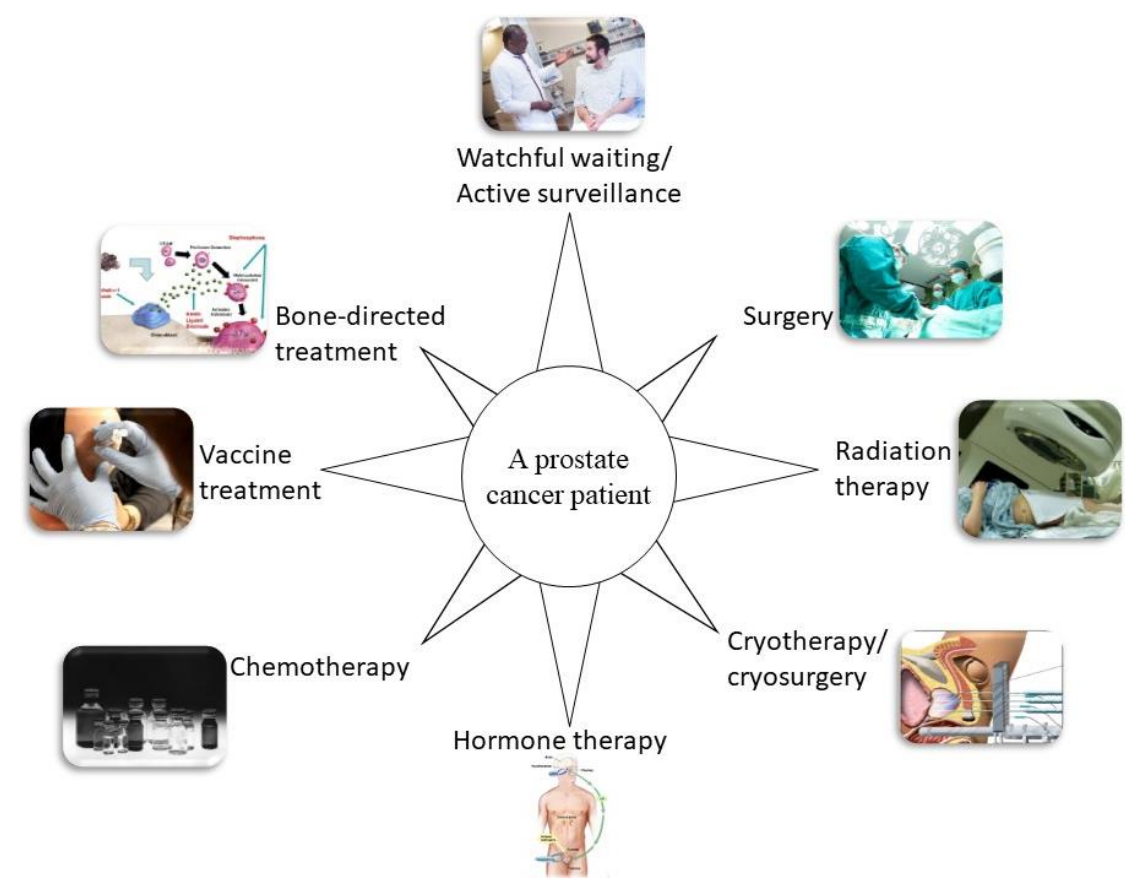

Fig. 1.1 An illustration of treatment options for patient diagnosed with prostate cancer.

The quality of radiation therapy in prostate cancer treatments is determined by many factors including the dose delivered to the organs at risks (OARs) such as the rectum, which could be affected by patient movement, internal motion of the organ, and patient set-up errors (Langen and Jones 2001; Muren et al. 2004). Figure 1.2 visualizes the interfractional shape variation of rectum and bladder with respect to the planning target volume (PTV) as determined by radiation oncologists. The rectal position uncertainties, which could cause toxicities (e.g., rectal bleeding, fecal incontinence), mainly comes from 
the rectal motion due to the changes in rectal filling (Lebesque et al. 1995; Roeske et al. 1995; M J Zelefsky et al. 2000; Michael J. Zelefsky et al. 2002; Foppiano et al. 2003). The two common methods used to study the rectal motion were tracking the changes in rectal volume (Muren et al. 2004; Michael J. Zelefsky et al. 1999) and evaluating the translation and rotation errors of the rectum (van Herk et al. 1995; Rijkhorst et al. 2009). Fontenla et al. (Fontenla et al. 2001), however, noted that the more complex problem of internal organ motion involve changes in the shape (shape variations) of the organ especially along the anterior direction of the rectum (Muren et al. 2004; Dias et al. 2011). Therefore, the shape variations of the rectum, especially along the anterior direction, need to be investigated.

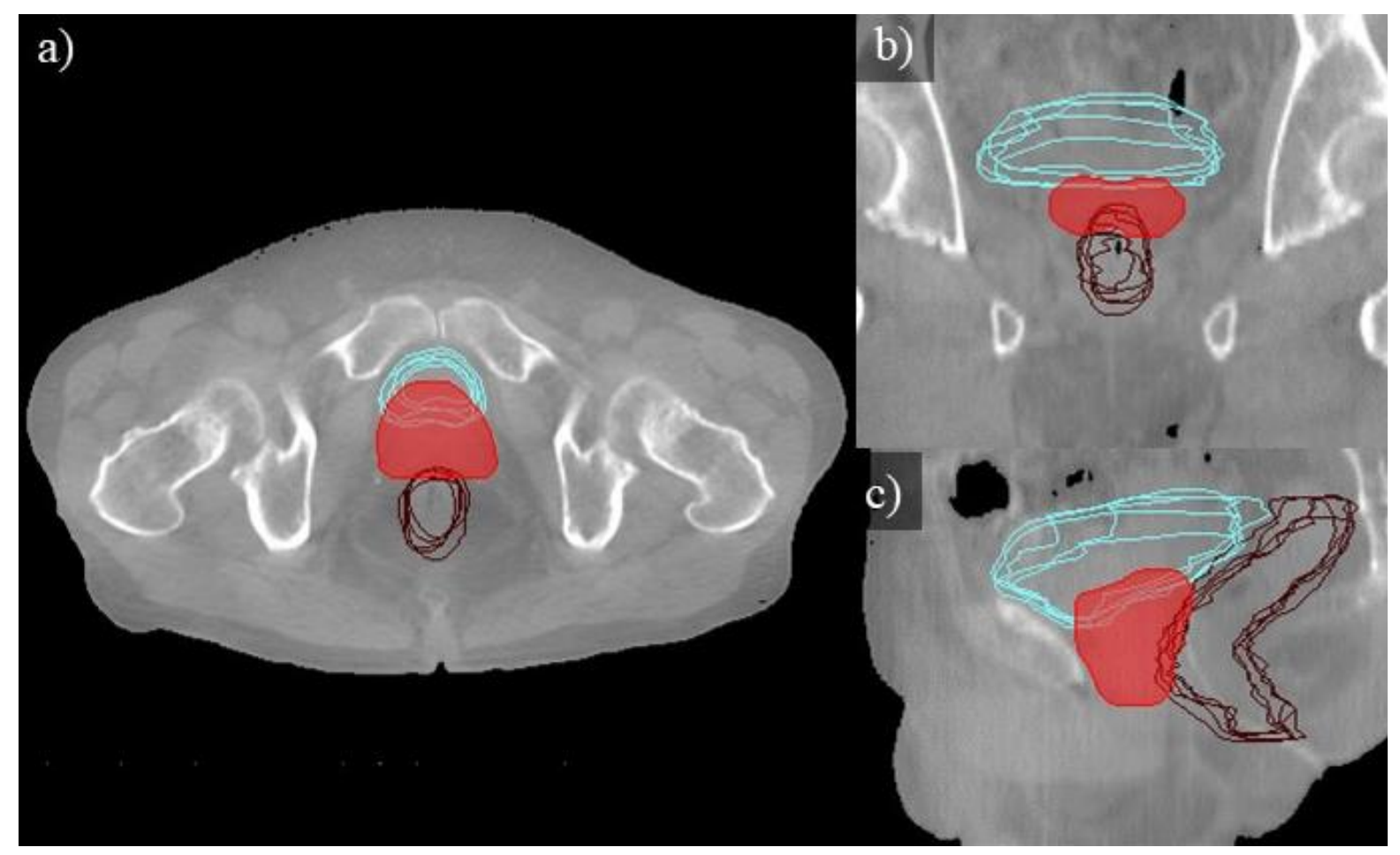

Fig. 1.2 A visualization of the interfractional shape variations of rectum (magenta contour) and bladder (cyan contour) with respect to the PTV (red contour), as delineated by radiation oncologists: a) axial view, b) coronal view, and c) sagittal view. Note that parts of the rectum and the bladder were overlapped with the PTV which could cause high dose exposure to both OARs.

The regions in which the rectum overlapped with the PTV along the anterior wall is defined as the rectum-overlap PTV (ROP) region. Figure 1.3 shows the visualization of the ROP region of a patient at a fraction as determined by radiation oncologists. The size of the ROP region, which was found to be correlated with the adverse effect to the rectum, is affected by the size and shape of the rectum and the 
PTV delineated by the radiation oncologist during the treatment planning process (Mattes et al. 2014). The dose-volume parameters higher than $60 \mathrm{~Gy}$, especially $V_{70}$ and $V_{75}$ of the rectum, were found to be significantly correlated with late rectal toxicity such as rectal bleeding (Prabhakar et al. 2016; Michalski et al. 2010). Therefore, the analysis of the ROP region is necessary to reduce the risk of toxicity to the rectum.

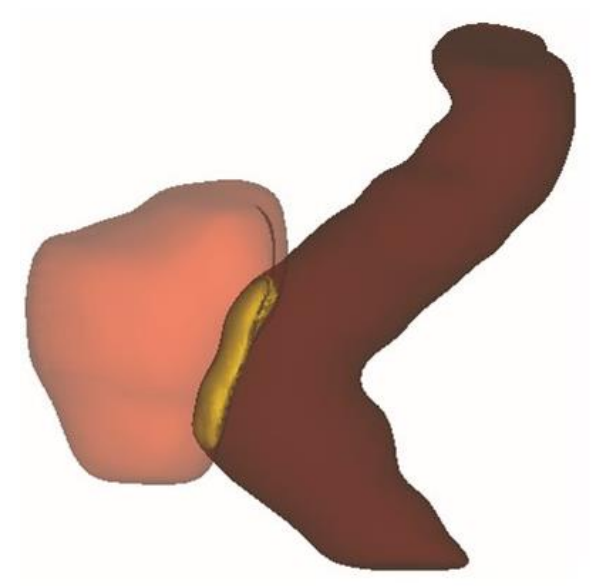

a)
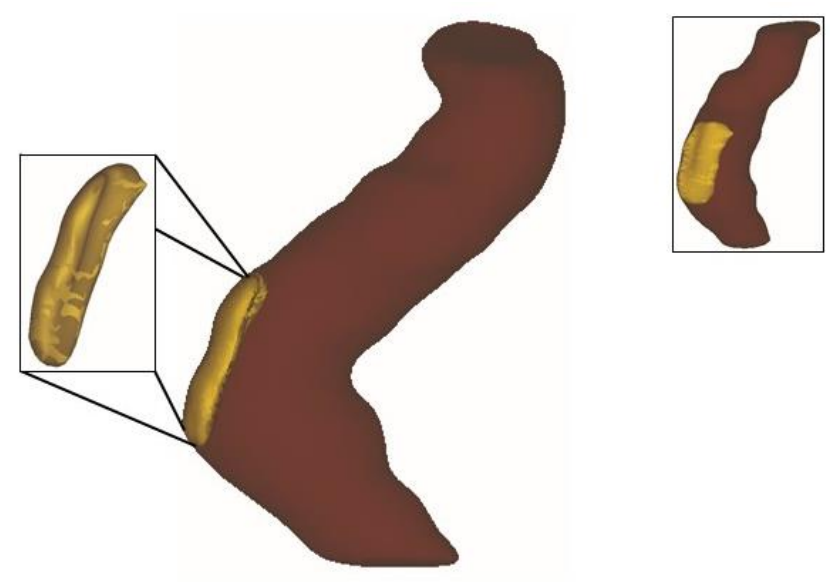

b)

Fig. 1.3 Visualizations of the ROP region of a patient at a fraction as determined by the radiation oncologists: (a) shows the PTV (red), rectum (brown), and the ROP region (yellow) as viewed from the LR direction, while (b) shows only the rectum and the ROP region which covers the anterior part of the rectum.

In order to dealt with the position uncertainties of the, the International Commission on Radiation Units and Measurements (ICRU) reports no. 62 (ICRU 1999) and 83 (ICRU 2010) introduced the concept of planning risk volume (PRV) margins. Figure 1.4 illustrates the application of PRV margin to the rectum structure for prostate cancer radiation therapy. The use of rectum PRV dosevolume histograms (DVHs) is recommended to predict acute rectal toxicity (Dias et al. 2011; Muren et al. 2005; Prabhakar et al. 2016). "Recipes" to determine the uniform PRV margins have been developed by McKenzie et al. (McKenzie, Van Herk, and Mijnheer 2002) and Stroom and Heijmen (Stroom and Heijmen 2006). However, the uniform PRV margins are inadequate to represent the actual rectal variations during treatment, as noted by McKenzie et al. and Prabhakar et al. (McKenzie, Van Herk, 
and Mijnheer 2002; Prabhakar et al. 2014). Therefore, an application of anisotropic PRV margins of the rectum should be considered.

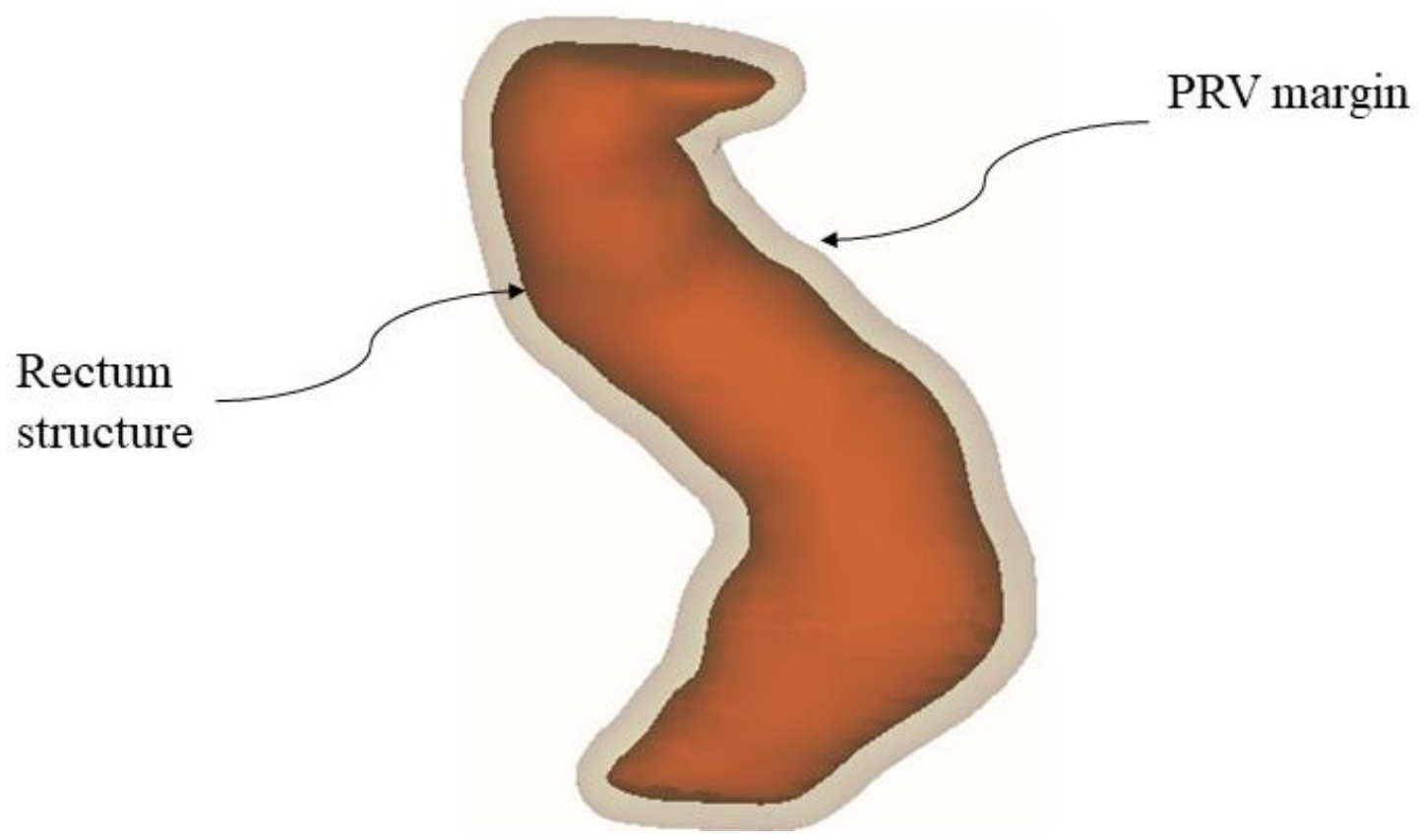

Fig. 1.4 An illustration of the application of PRV margin to rectum structure for prostate cancer radiation therapy.

There have been three studies that dealt with the shape variations of the rectum. Hoogeman et al. analyzed the quantification of local rectal wall displacements by calculating local systematic and random errors of the rectum along three directions where they unfolded the outer surface of the delineated rectal wall and projected the 3-space coordinates of each surface element to a 2D map (Hoogeman et al. 2002). Sohn et al. investigated the correlated motion of adjacent organ structures between prostate, bladder and rectum which were parametrized by using sets of corresponding surface points and calculated the displacements between surface points at each fraction (Sohn et al. 2005). They did not calculate the systematic and random errors that could be used in determining anisotropic PRV margins. Brierley et al. investigated the determination of the PTV based on the rectal shape variations by using finite element modeling (Brierley et al. 2011). They did not investigate the geometric errors related to the determination of PRV margins. 
None of the previously mentioned studies, including ICRU, investigated directly the shape variations of the rectum along each anatomical direction separately (anterior, posterior, superior, inferior, left and right). The investigation along separate anatomical directions is indispensable for determining the anisotropic PRV margins. There have been also no studies on the systematic and random errors of the region in which the rectum overlapped with the PTV along the anterior wall (referred to as rectum-overlap-PTV region, ROP regions), even though the shape variations of the ROP regions may cause the regions to be included in high dose distributions which can lead to rectum toxicities.

\subsection{Purpose}

The purpose of this study was to investigate the anisotropic shape variations of the rectum and the ROP regions for prostate cancer radiation therapy along separate anatomical direction (anterior, posterior, superior, inferior, left and right). 


\section{Chapter 2 Basic theory}

\subsection{Geometrical uncertainties in radiation therapy}

High geometrical accuracy is a prerequisite for a safe clinical application of radiation therapy (Van Herk 2004). The accuracy is affected by uncertainties, which came from the variability of organ delineations (observer errors), patient positioning (setup errors), and variation of organ shape (organ motion/internal errors). The uncertainties could cause differences between intended and actual delivered dose distribution during treatment (Arimura et al. 2017; Stroom et al. 1999). Several attempts have been made to reduce these uncertainties including the use of modern radiotherapy equipment and techniques, especially in terms of machine-related geometrical errors. However, residual uncertainties which came from organ motions always remains (Stroom and Heijmen 2002; Van Herk 2004; Arimura et al. 2017).

Geometrical uncertainties have to be considered when constructing the treatment plan (Stroom and Heijmen 2002). Therefore, the measurement of errors, defined as the deviations between planned and executed treatment, needs to be performed. However, the errors that the patient will experience in future treatments are not known. In such situation, the measured errors data in a group of similar patients, described by using standard deviations of systematic and random errors, may be used to infer the errors that might be experienced by a new patient (Stroom and Heijmen 2002; Van Herk 2004).

\subsubsection{Systematic and random errors}

Stroom and Heijmen (2002), defined the systematic errors of an organ for individual patients as the mean deviations between the organ's position in the treatment plan and the mean organ's position in the fractionated treatment, and the random errors as fraction-to-fraction variations around the mean deviations. The errors measured in a group of similar patients are then described by a population standard deviation. The population standard deviations of the systematic errors indicate the patient-topatient variation in the systematic deviation from the planning situation. Meanwhile, the population standard deviations of the random errors is the mean of the observed random standard deviations for the patients in the group (Stroom and Heijmen 2002). 


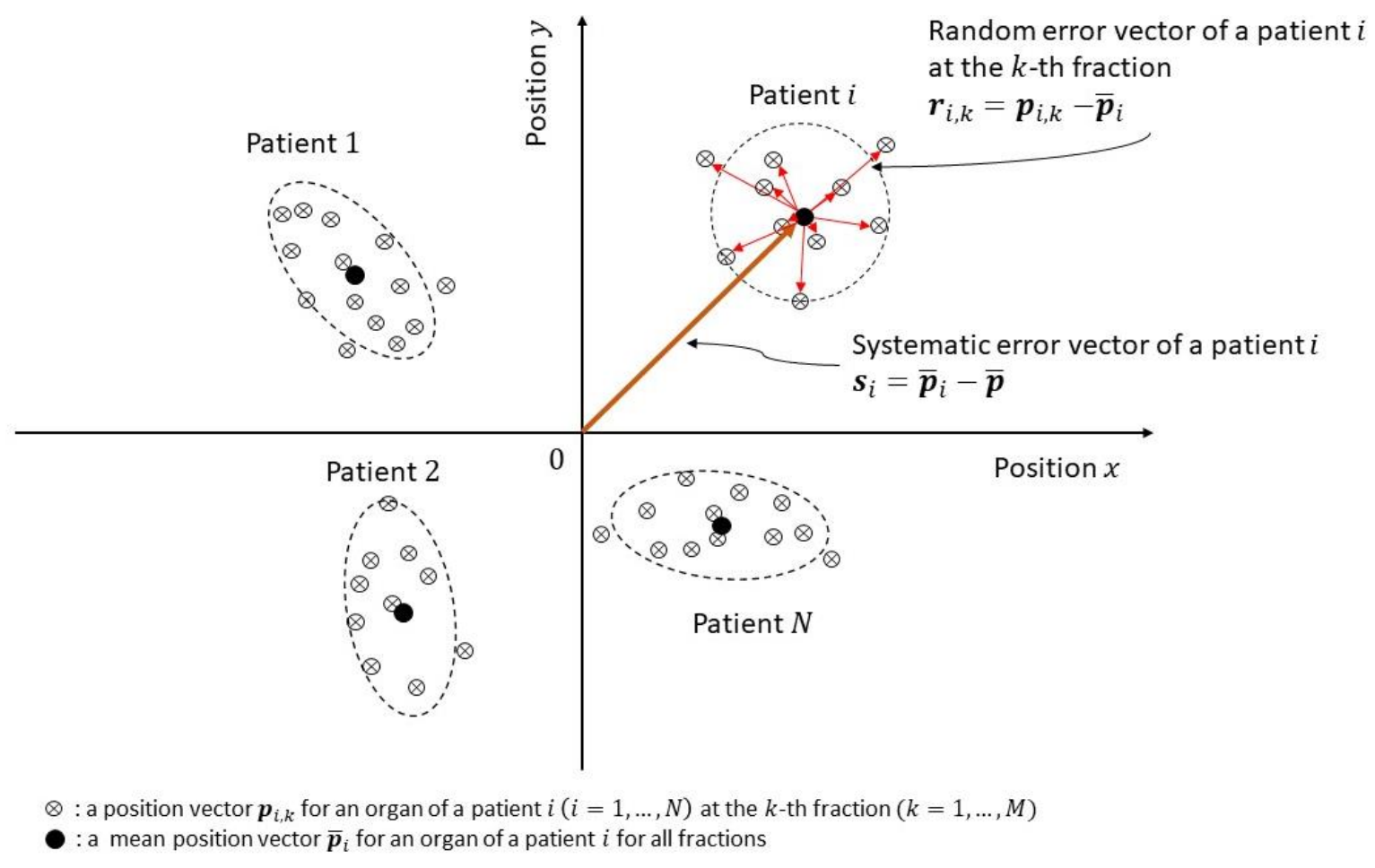

Fig. 2.1 An illustration of the systematic and random errors of an organ for a population of $N$ patients.

The calculation of the standard deviations of systematic and random errors were described by Arimura et al. (2017). Figure 2.1 shows an illustration of the systematic and random errors of an organ for a population of $N$ patients. Let an organ position vector of a patient $i(i=1, \ldots, N)$ at a $k$-th fraction $(k=1, \ldots, M)$ be $\boldsymbol{p}_{i, k}$. The organ's mean position vector $\overline{\boldsymbol{p}}_{i}$ of a patient $i$ for all fractions can be calculated as

$$
\overline{\boldsymbol{p}}_{i}=\frac{1}{M} \sum_{k=1}^{M} \boldsymbol{p}_{i, k}
$$

Then, a mean deviation vector $\overline{\boldsymbol{p}}$ of the organ's mean position vector $\overline{\boldsymbol{p}}_{i}$ for all patients can be calculated by

$$
\overline{\boldsymbol{p}}=\frac{1}{N} \sum_{i=1}^{N} \overline{\boldsymbol{p}}_{i}
$$

which could be close to a zero vector. The systematic error vector $\boldsymbol{s}_{i}$ of a patient $i$ can be calculated as 


$$
\boldsymbol{s}_{i}=\overline{\boldsymbol{p}}_{i}-\overline{\boldsymbol{p}}=\left(s_{i}(x), s_{i}(y), s_{i}(z)\right) .
$$

where $s_{i}(x), s_{i}(y)$, and $s_{i}(z)$ represent the $x, y$, and $z$ components of the systematic error vector of a patient $i$, respectively. Therefore, the population systematic error along a specific direction, e.g. along $x$ direction $(\varepsilon(x)$ ), can be defined as (Stroom and Heijmen 2002; Strbac and Jokic 2013; Arimura et al. 2017)

$$
\varepsilon(x)=\sqrt{\frac{1}{N} \sum_{i=1}^{N} s_{i}(x)^{2} .}
$$

On the other hand, the random vector $\boldsymbol{r}_{i, k}$ for the $k$-th fraction of the $i$-th patient is defined as

$$
\boldsymbol{r}_{i, k}=\boldsymbol{p}_{i, k}-\overline{\boldsymbol{p}}_{i}=\left(r_{i, k}(x), r_{i, k}(y), r_{i, k}(z)\right)
$$

where $r_{i, k}(x), r_{i, k}(y)$, and $r_{i, k}(z)$ represent the $x, y$, and $z$ components of the random error vector of a patient $i$ at fraction $k$, respectively. Therefore, the random error of a patient $i$ along the $x$ direction $\left(\sigma_{i}(x)\right)$ can be calculated as

$$
\sigma_{i}(x)=\sqrt{\frac{1}{M} \sum_{k=1}^{M} r_{i, k}(x)^{2}} .
$$

The random error for all patients along the $x$ direction $(\sigma(x))$ can be calculated as

$$
\sigma(x)=\sqrt{\frac{1}{N} \sum_{i=1}^{N} \sigma_{i}(x)^{2}} .
$$

The systematic and random errors along the $y$ and $z$ directions are calculated in the same way (Arimura et al. 2017). 


\subsubsection{Planning risk volume (PRV) margin}

ICRU-50, 62, and 83 recommend the inclusion of geometrical uncertainties in radiation treatment planning by adding a safety margin surrounding the target volume and OARs (Stroom and Heijmen 2002; ICRU 1999; ICRU 2010; Arimura et al. 2017). ICRU-83 stated that uncertainties and variations of the OAR during treatment must be considered to avoid serious complications (i.e. toxicity). Therefore, a PRV margin needs to be added to the OAR to ensure that the absorbed dose delivered to the OAR match the prescription constraints (ICRU 2010).

The PRV margin can be determined by using similar principle as PTV determination. ICRU83 recommends the calculation of PRV by using the systematic and random uncertainties of the OAR. Several researchers have attempted to formulate the "recipe" for the calculation of PRV, however the most commonly used formula was produced by McKenzie et al. (2002). In their study, they conclude that for a uniform margin surrounding the OAR, the size of the margin can be calculated as

$$
\mathrm{PRV} \text { margin }=1.3 \varepsilon+0.5 \sigma,
$$

where $\varepsilon$ is the standard deviation of the combined systematic errors and $\sigma$ represents the standard deviation of the combined random errors. The width $1.3 \varepsilon$ will ensure that the mean position of the edge of the OAR will be encompassed by this margin along any single direction in $90 \%$ of treatment plans (McKenzie, Van Herk, and Mijnheer 2002).

\subsection{Point distribution model (PDM)}

A statistical shape model of an organ is commonly used for the prediction of fraction-to-fraction variations for a populations of patient in computational approaches in radiation treatment planning (Arimura et al. 2017). The use of shape models, i.e. point distribution model (PDM), which describe and label the expected structure of human organs are beneficial for analysing the shape variation of organs (T. Cootes and Taylor 2001; Shibayama et al. 2017).

PDM represents shapes by distributing a set of points across the surface and concatenated the coordinates for all the points to one vector (T. F. Cootes and Taylor 1992; Heimann and Meinzer 2009). PDM represent shapes by distributing a set of points across the surface and concatenated the coordinates 
for all the points to one vector (Heimann and Meinzer 2009; T. F. Cootes et al. 1992). The set of points can be extracted from volume data by, e.g. the marching cubes algorithm (Lorensen and Cline 1987). The surface triangulation of the volume data produced surface points (vertex) and faces of the surfaces, in which each vertex is connected to other vertex by edges, as shown in Fig. 2.2. The surfaces can be simplified by reducing the vertices to obtain a faster computing time while preserving the quality of the surface representation, by using a quadric error metrics method (Garland and Heckbert 1997).

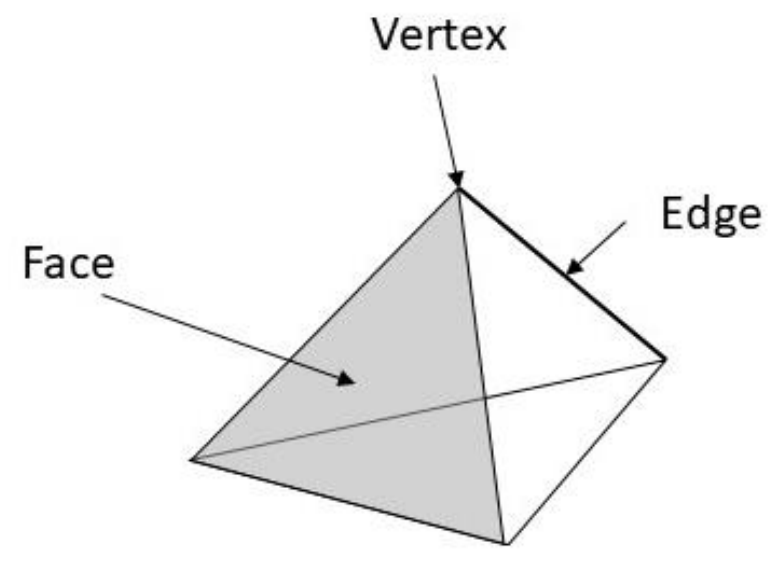

Fig. 2.2 An illustration of vertex, edge, and face of a triangulated surface.

\subsubsection{Surface triangulation by the marching cubes algorithm}

The marching cubes algorithm creates a polygonal representation of constant density surfaces from a volume data (Lorensen and Cline 1987). The algorithm first locates the surface corresponding to a userspecified value. The surfaces were located by using a logical cube created from eight pixels; four each from two adjacent slices. The algorithm then determines the surface intersection in a cube by assigning binary values to a cube's vertex, based on the data value at the vertex. Since there are eight vertices in each cube and two states ( 1 or 0$)$, inside and outside, it created a $2^{8}=256$ possibilities of how a surface intersecting a cube. However, by considering two different symmetries of the cube, the possibilities can be reduced to 14 patterns. Figure 2.3 shows the triangulation for the 14 patterns (Long and Nagamune 2015). The algorithm move to the next cube until all the surface pixels are covered (Lorensen and Cline 1987). 


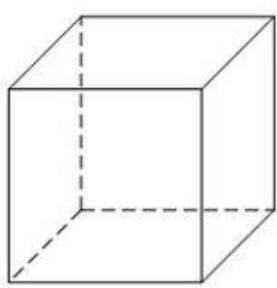

case 0

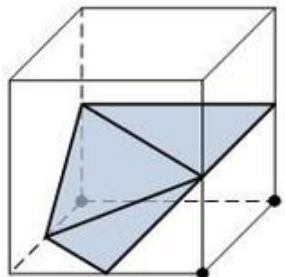

case 5

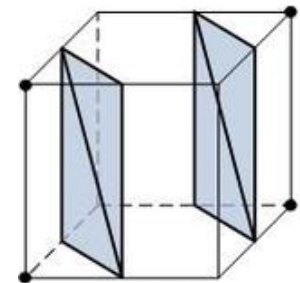

case 10

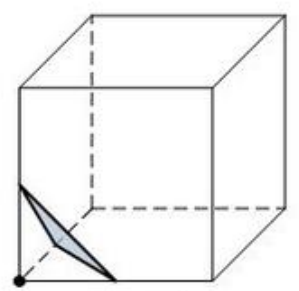

case 1

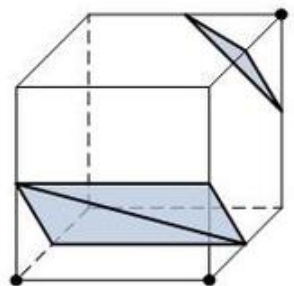

case 6

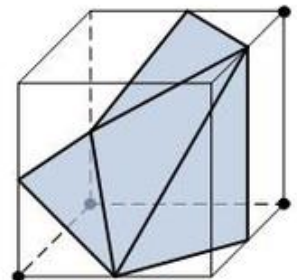

case 11

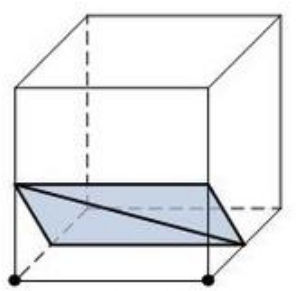

case 2

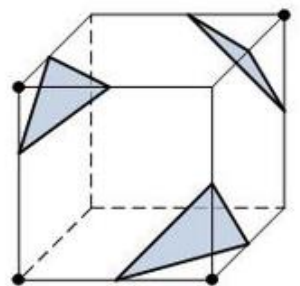

case 7

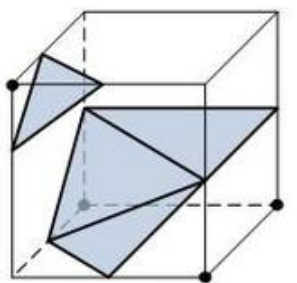

case 12

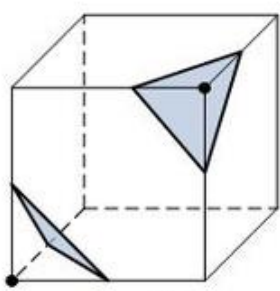

case 3

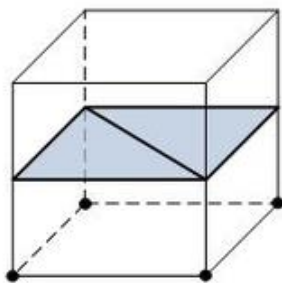

case 8

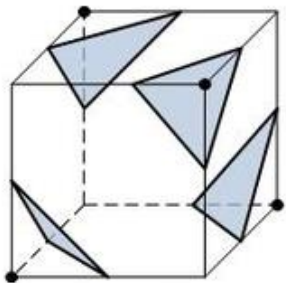

case 13

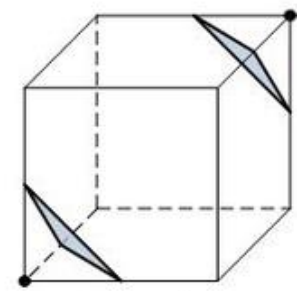

case 4

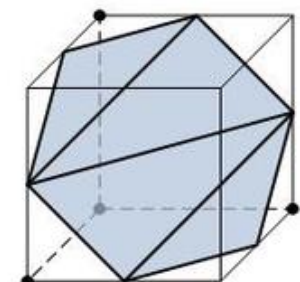

case 9

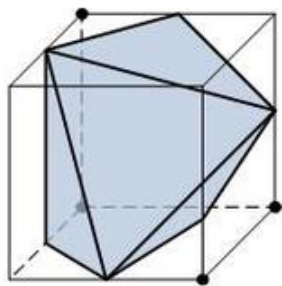

case 14

Fig. 2.3 Illustrations of the triangulation patterns used in marching cubes algorithm (Long and Nagamune 2015).

\subsubsection{Surface simplification by the quadric error metrics method}

It is often necessary to produce a simpler version of a computer-generated model in order to lower the computational cost. The quadric error metrics method aims to generate a simplified surface model (approximation of the original input) from a polygonal surface model (Garland and Heckbert 1997). The approximation will satisfy some given criterion such as a desired face count or a maximum tolerable error.

The quadric error metrics algorithm is based on the iterative contraction of vertex pairs. Let $M_{n}$ be the input polygonal surface model consisting of a set of vertices $\left(\mathbf{v}_{1}, \mathbf{v}_{2}, \ldots, \mathbf{v}_{k} ; k=\right.$ number of vertices). A pair of vertex $\left(\mathbf{v}_{1}, \mathbf{v}_{2}\right)$ will be valid for contraction $\left(\mathbf{v}_{1}, \mathbf{v}_{2}\right) \rightarrow \overline{\mathbf{v}}$, where $\overline{\mathbf{v}}$ denotes the new position of the contraction result, if either of the following criterion is met:

1. $\left(\mathbf{v}_{1}, \mathbf{v}_{2}\right)$ is an edge, or 
2. $\left\|\mathbf{v}_{1}-\mathbf{v}_{2}\right\|<t$

where $t$ denotes the threshold parameter. Figure 2.4(a) illustrates the pair contraction for an edge pair while Figure 2.4(b) illustrates the pair contraction for non-edge pair.

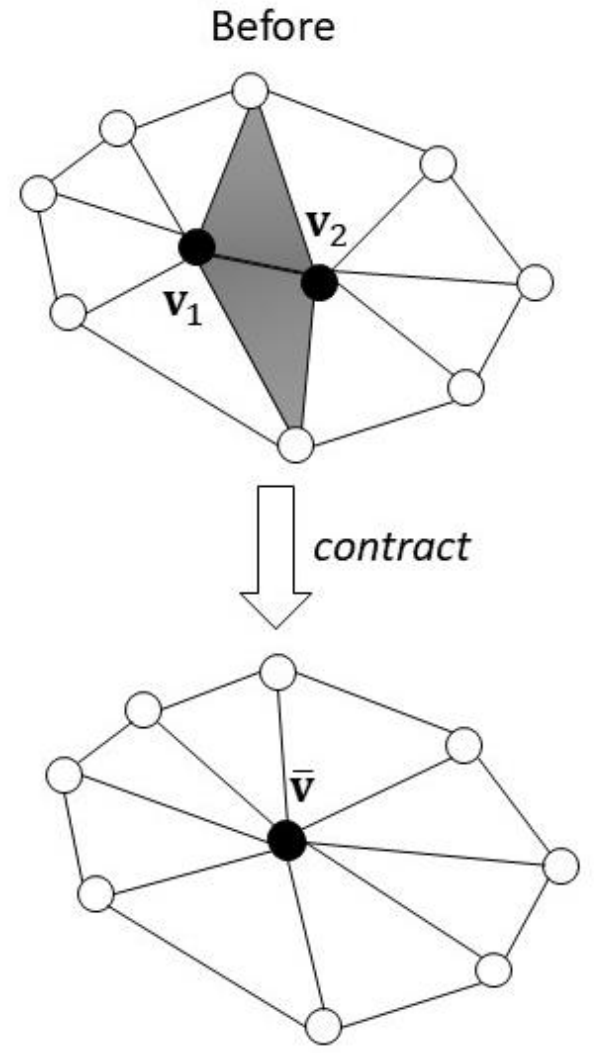

After

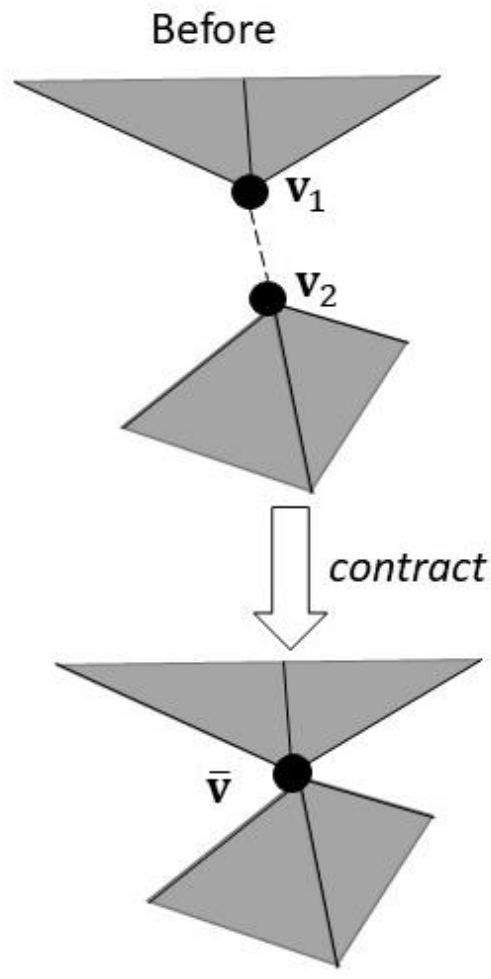

After

a)

b)

Fig. 2.4 Illustrations of pair contraction in the quadric error metrics algorithm: a) for an edge pair, and b) for a non-edge pair.

The cost of contraction is defined to select a contraction which will be performed during a given iteration by associating a symmetric $4 \times 4$ matrix $\mathbf{Q}$ with each vertex. The error at vertex $\mathbf{v}=$ $\left[\begin{array}{llll}\boldsymbol{v}_{\boldsymbol{x}} & \boldsymbol{v}_{\boldsymbol{y}} & \boldsymbol{v}_{\boldsymbol{z}} & \mathbf{1}\end{array}\right]^{\mathbf{T}}$ can be defined as

$$
\Delta(\mathbf{v})=\Delta\left(\left[\begin{array}{llll}
v_{x} & v_{y} & v_{z} & 1
\end{array}\right]^{\mathrm{T}}\right)=\sum_{\mathbf{p} \in \operatorname{planes}(\mathrm{v})}\left(\mathbf{p}^{\mathrm{T}} \mathbf{v}\right)^{2}
$$


where $\mathbf{p}=\left[\begin{array}{lll}a & b & c\end{array}\right]^{\mathrm{T}}$ represents the plane associated with each vertex defined by the equation $a x+$ $b y+c z+d=0$ where $a^{2}+b^{2}+c^{2}=1$. This approximation was obtained by assuming that each vertex in the original model is a solution of the intersection of a set of planes of the triangles that meet at each vertex. The quadratic form of Eq. (2.9) can be rewritten as

$$
\begin{aligned}
\Delta(\mathbf{v}) & =\sum_{\mathbf{p} \in \operatorname{planes}(\mathrm{v})}\left(\mathbf{v}^{\mathrm{T}} \mathbf{p}\right)\left(\mathbf{p}^{\mathrm{T}} \mathbf{v}\right) \\
& =\sum_{\mathbf{p} \in \operatorname{planes}(\mathrm{v})} \mathbf{v}^{\mathrm{T}}\left(\mathbf{p} \mathbf{p}^{\mathrm{T}}\right) \mathbf{v} \\
& =\mathbf{v}^{\mathrm{T}}\left(\sum_{\mathbf{p} \in \operatorname{planes}(\mathrm{v})} \mathbf{K}_{\mathrm{p}}\right) \mathbf{v}
\end{aligned}
$$

where $\mathbf{K}_{\mathrm{p}}$ is the fundamental error quadric matrix which can be used to find the squared distance of any point in space to the plane $\mathbf{p}$. The fundamental error quadrics matrices can be summed up together and represent an entire set of planes by a single matrix $\mathbf{Q}$. A fundamental quadric error matrix can be defined as

$$
\mathbf{K}_{\mathrm{p}}=\mathbf{p p}^{\mathrm{T}}=\left[\begin{array}{llll}
a^{2} & a b & a c & a d \\
a b & b^{2} & b c & b d \\
a c & b c & c^{2} & c d \\
a d & b d & c d & d^{2}
\end{array}\right]
$$

Therefore, the quadric error metrics method can be summarized as follows:

Step 1. Compute the $\mathbf{Q}$ matrices for all initial vertices.

Step 2. Selection of all valid pairs.

Step 3. Computation of the optimal contraction target $\overline{\mathbf{v}}$ for each valid pair $\left(\mathbf{v}_{1}, \mathbf{v}_{2}\right)$. The error $\overline{\mathbf{v}}^{\mathrm{T}}\left(\mathbf{Q}_{1}+\right.$ $\left.\mathbf{Q}_{2}\right) \overline{\mathbf{v}}$ of the target vertex is calculated as the cost of contracting the pair.

Step 4. List all pairs with the pair having minimum cost placed at the top of the list.

Step 5. Iteratively contract the pair $\left(\mathbf{v}_{1}, \mathbf{v}_{2}\right)$ starting from the top and update the costs for all valid pairs involving $\mathbf{v}_{1}$. 


\subsubsection{PDM correspondences determination by using thin-plate spline robust point matching (TPS-RPM) algorithm}

Modelling the statistics of a class of shapes requires a set of shapes with well-defined correspondences (Heimann and Meinzer 2009). In principle, the algorithm that compute correspondences between two shapes is actually perform a registration between the shapes. One of the algorithm commonly used for computing the correspondence between two point sets is thin-plate spline robust point matching (TPSRPM) algorithm.

TPS-RPM algorithm is a non-rigid point matching algorithm that minimizes the distances between two point sets based on deterministic annealing and softassign algorithms. The general idea of the algorithm is to incorporate the thin-plate spline as a non-rigid mapping method to the robust point matching algorithm which is known to be only using affine and piecewise-affine mappings (Chui and Rangarajan 2003). Let $X$ be a set of points from a source surface consisting of $\left\{x_{a}\right\}$ where $(a=$ $1,2, \ldots, H ; H$ : number of points) which correspondences with $V$, a set of points from a target surface consisting of $\left\{v_{b}\right\}$ where $(b=1,2, \ldots, D ; D$ : number of points), are to be determined. The mapping of a point $x_{a}$ to a new location by a non-rigid transformation is represented by a general function $f$. The goal of TPS-RPM is to minimize the following cost function based on the mapping function $f\left(x_{a}\right)$ between corresponding sets $\left\{x_{a}\right\}$ and $\left\{v_{b}\right\}$ :

$$
\min _{Z, f} \sum_{b=1}^{D} \sum_{a=1}^{H} z_{a b}\left\|v_{b}-f\left(x_{a}\right)\right\|^{2}+\lambda\|f\|^{2},
$$

where $Z$ is the correspondence matrix consisting of $\left\{z_{a b}\right\},\|f\|$ is the L2 norm, $\lambda$ is the hyper-parameter that trades off between the registration and regularization of $f$, and $\|f\|^{2}$ is the TPS regularizer which calculates the bending cost of $f$ and acts as a smoothness measure (Chui and Rangarajan 2003; S. H. Huang et al. 2015).

\subsubsection{Analysis of geometric uncertainties using PDM}

Computational anatomical approaches based on PDMs are promising tools for the determination of safety margins in radiation treatment planning, since we can analyze the motion of each point 
displacement on the surface of an organ for a population of patients at a number of treatment fractions (Arimura et al. 2017). The method can be applied to CTV (Shibayama et al. 2017) as well as to OARs (Sohn et al. 2005; Haekal et al. 2018).

Figure 2.5 illustrates a PDM of an object. Let $\boldsymbol{p}_{i, j}(k)=\left(p_{i, j, x}(k), p_{i, j, y}(k), p_{i, j, z}(k)\right)^{\mathrm{T}}$ be a 3D position vector of surface point (vertex) $k(k=1, \ldots, M)$ on the object surface $\boldsymbol{S}_{i, j}$ for the $i$-th patient $(i=1, \ldots, N)$ at the $j$-th fraction $(j=1, \ldots, F ; j=1:$ reference fraction). Here, $x, y$, and $z$ represent the right-left (RL), anterior-posterior (AP), and superior-inferior (SI) directions, respectively. The surface (shape) of an object can be defined as a matrix (a set of vertex vectors) as follows:

$$
\boldsymbol{S}_{i, j}=\left(\left(\boldsymbol{p}_{i, j}(1) \boldsymbol{p}_{i, j}(2) \ldots \boldsymbol{p}_{i, j}(k) \ldots \boldsymbol{p}_{i, j}(M)\right)\right)
$$

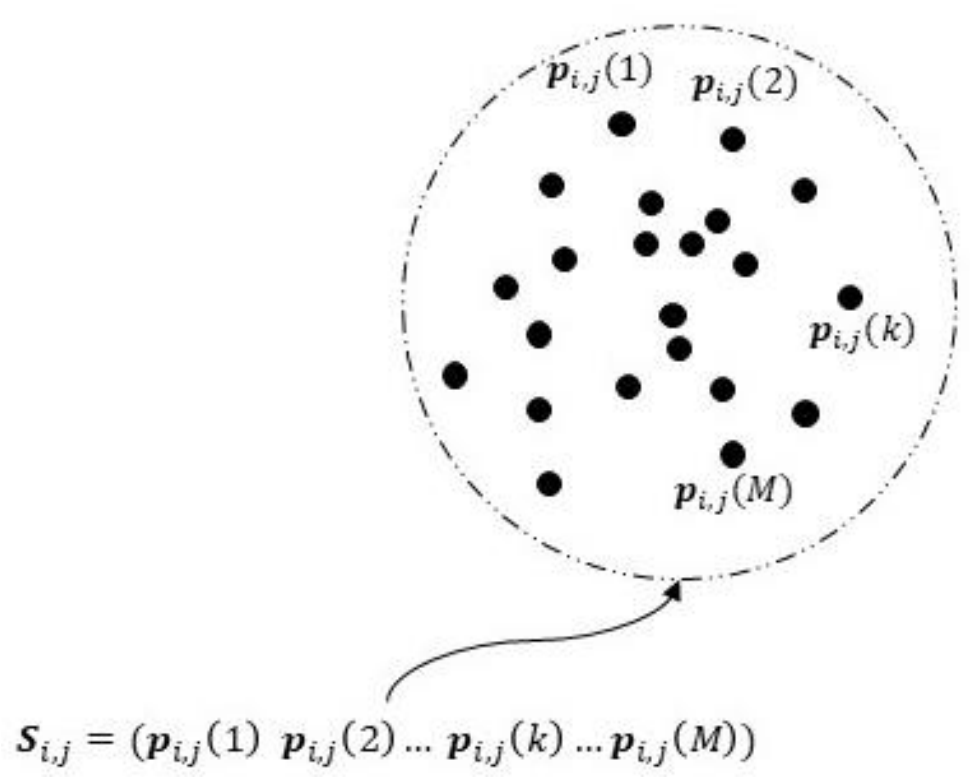

Fig. 2.5 An illustration of a point distribution model of an object.

The PDMs can be used to calculate the systematic and random errors of the vertices (van der Wielen et al. 2008). Let a displacement matrix of the transformed surface $\boldsymbol{D}_{i, j}^{(1)}=$ $\left(\boldsymbol{d}_{i, j}(1) \boldsymbol{d}_{i, j}(2) \ldots \boldsymbol{d}_{i, j}(k) \ldots \boldsymbol{d}_{i, j}(M)\right)$ be for the $j$-th fraction of the $i$-th patient from the surface $\boldsymbol{S}_{i, 1}$ for the first (reference) fraction. The displacement matrix is derived from 


$$
\boldsymbol{D}_{i, j}^{(1)}=\boldsymbol{T}_{i, j}^{(1)} \boldsymbol{S}_{i, j}^{(1)}-\boldsymbol{S}_{i, 1}
$$

where $\boldsymbol{T}_{i, j}^{(1)}$ is the non-rigid transformation matrix which used to register each surface $\boldsymbol{S}_{i, j}$ with a reference surface $\boldsymbol{S}_{i, 1}$. The average surface $\overline{\boldsymbol{S}}_{i}$ for all fractions of the $i$-th patient is obtained from

$$
\overline{\boldsymbol{S}}_{i}=\boldsymbol{S}_{i, 1}+\overline{\boldsymbol{D}}_{i}
$$

where

$$
\overline{\boldsymbol{D}}_{i}=\frac{1}{F} \sum_{j=1}^{F} \boldsymbol{D}_{i, j}
$$

and

$$
\overline{\boldsymbol{D}}_{i}=\left(\overline{\boldsymbol{d}}_{i}(1) \overline{\boldsymbol{d}}_{i}(2) \ldots \overline{\boldsymbol{d}}_{i}(k) \ldots \overline{\boldsymbol{d}}_{i}(M)\right)
$$

The displacement matrix $\boldsymbol{D}_{i}^{(a)}$ of the transformed average surface $\boldsymbol{T}_{i}^{(a)} \overline{\boldsymbol{S}}_{i}$ of the $i$-th patient from the average surface for all fractions of the first patient $\overline{\boldsymbol{S}}_{i}$ can be calculated as

$$
D_{i}^{(a)}=\boldsymbol{T}_{i}^{(a)} \overline{\boldsymbol{S}}_{i}-\overline{\boldsymbol{S}}_{i}
$$

The average surface $\overline{\boldsymbol{S}}$ for all fractions of all patients can be calculated as

$$
\overline{\boldsymbol{S}}=\overline{\boldsymbol{S}}_{1}+\boldsymbol{D}^{(a)}
$$

where

$$
\boldsymbol{D}^{(a)}=\frac{1}{N} \sum_{i=1}^{N} \boldsymbol{D}_{i}^{(a)}
$$

Therefore, the systematic displacement $\varepsilon_{i}(k)$ along the surface normal vector $\boldsymbol{n}_{i}(k)$ at the $k$ th vertex, which is distributed on the average surface $\overline{\boldsymbol{S}}_{i}$ of the $i$-th patient, can be calculated as (van der Wielen et al. 2008) 


$$
\varepsilon_{i}(k)=\frac{1}{F-1} \sum_{j=1}^{F}\left(\boldsymbol{d}_{i, j}^{(1)}(k), \boldsymbol{n}_{i}(k)\right),
$$

where (',') denotes the inner product. The corresponding SDs of the random displacement along the surface normal vector can be obtained by

$$
\sigma_{i}(k)=\frac{1}{F-1} \sum_{j=1}^{F}\left(\boldsymbol{d}_{i, j}^{(1)}(k)-\overline{\boldsymbol{d}}_{i}(k), \boldsymbol{n}_{i}(k)\right)^{2}
$$




\section{Chapter 3 Computational analysis of interfractional anisotropic shape variations of the rectum in prostate cancer radiation therapy}

\subsection{Clinical study}

This study was performed with the approval of the Institutional Review Board of our university hospital. The clinical data used in this study were obtained from 11 patients (range: 60-75 years; median age: 64 years; stage T1-T3a, N0, M0), who had undergone intensity-modulated radiation therapy (IMRT) for prostate cancer. The planning computed tomography (CT) images were acquired from a CT scanner (Mx 8000, Philips, Amsterdam, Netherlands) with $512 \times 512$-pixel dimensions, $0.98 \mathrm{~mm}$ in-plane pixel size and $2.0 \mathrm{~mm}$ slice thickness. Each patient received a dose of 76 Gy at 38 fractions using an accelerating voltage of $10 \mathrm{MV}$ on a linear accelerator (Varian Medical Systems Inc., Palo Alto, USA).

The analyses of systematic and random errors of interfractional anisotropic shape variations of the rectum were derived from contours delineated on the planning CT and cone-beam CT (CBCT) images at 80 fractions of 11 patients. CBCT scans were performed just before irradiation at 5-9 fractions (mean: 7.3) of each patient. The CBCT data were used for correcting target localization at each fraction and only acquired at the beginning of the week to reduce the dose received by the patients. A kilovoltage CBCT scanner (On-Board-Imager, Varian Medical Image Systems Inc., Palo Alto, USA) was used to perform the scans which produced images with $384 \times 384$-pixel dimensions, $1.17 \mathrm{~mm}$ in-plane pixel size and $2.5 \mathrm{~mm}$ slice thickness. The delineations of the rectum contours were based on a consensus between a radiation oncologist (S.O.) and a medical physicist (T.H.) using a commercially available radiation treatment planning (RTP) system (Eclipse version 6.5 and 8.1, Varian Medical System Inc., Palo Alto, USA).

\subsection{Pre-processing}

The original planning CT and CBCT images were converted into isotropic images with an isovoxel size of $1.17 \mathrm{~mm}$ using a cubic interpolation method. The rectum structures delineated on both planning CT and CBCT images were also extracted and converted using a shape-based interpolation method (Herman, Zheng, and Bucholtz 1992). 
In this study, we focused on the errors introduced by the inter-fractional organ motions of the rectum. To reduce the effect of intraobserver variation, uniform-length rectums were used for all cases. The reference length was equal to the shortest length $(7 \mathrm{~cm})$ of a rectum between starting and ending slices (around anus to sigmoid positions) delineated on the CBCT images among all cases (Hoogeman et al. 2002).

\subsection{Calculations of errors due to shape variations}

This study evaluated the local errors of the shape variations by dealing with them separately along each axis, as illustrated in Fig. 3.1. The $x, y$ and $z$ axes are each separated along their positive and negative directions corresponding to the anterior, posterior, superior, inferior, left and right directions. The local errors were calculated as the displacements of position vectors at points on 3D surfaces of the rectum (Fig. 3.2). The rotation errors of the rectum were included as shape variations since the errors also introduced the displacements of the surface points (Shibayama et al. 2017).

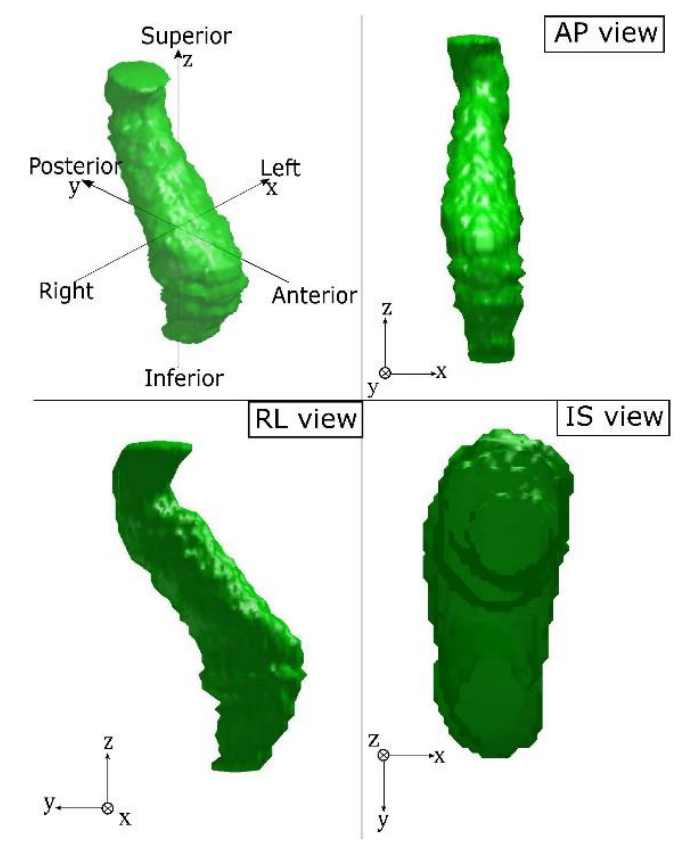

Fig. 3.1 Illustrations of anatomical directions describing rectum orientation. The " $x "$, " $y$ " and "z" axes correspond to the right-left (RL), anterior-posterior (AP) and inferior-superior (IS) directions, respectively. 


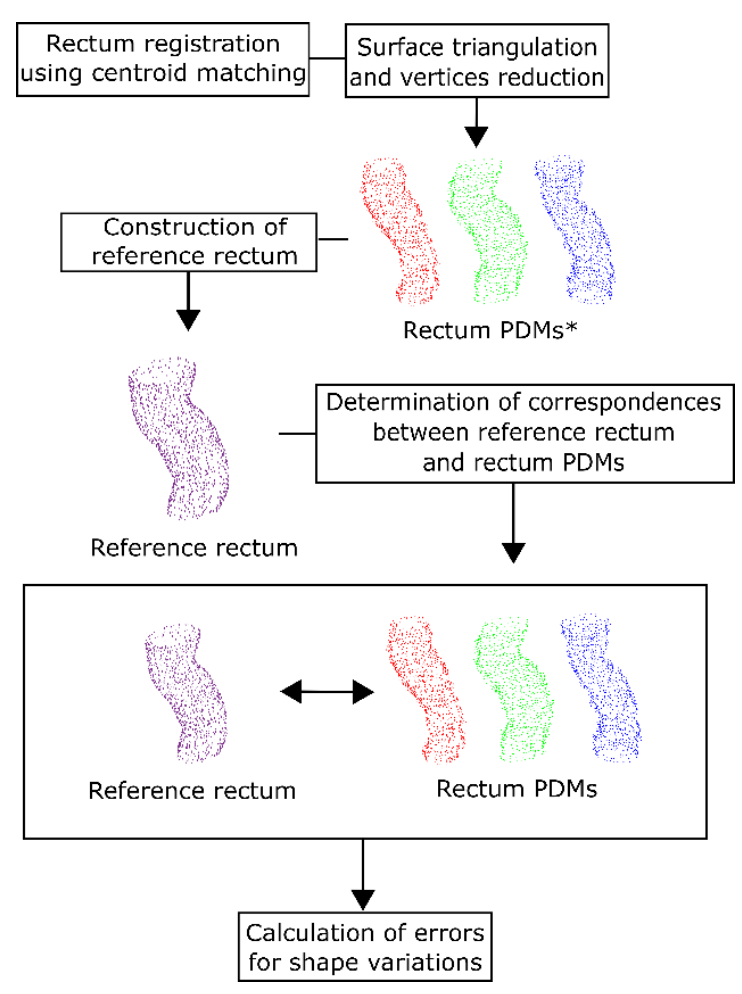

Fig. 3.2 A flowchart to calculate systematic and random errors of the rectum due to shape variations using a statistical point distribution models (PDMs).

The flowchart to calculate errors due to shape variations from planning CT and CBCT images were described in Fig. 3.2. First, all rectums were registered using a centroid matching technique. Then, the surfaces of the registered rectums were triangulated using a marching cubes algorithm to obtain a 3D surfaces of the rectum (Lorensen and Cline 1987). The produced surfaces consisted of many vertices, which were reduced to a similar number of vertices $(\sim 1000)$ using a quadric error metric method (Garland and Heckbert 1997). Thin-plate spline robust point matching (TPS-RPM) algorithm was applied to determine the correspondences between two surface structures.

In this study, the systematic error of a patient due to shape variations was defined as the average deviation of a reference rectum from the planning rectum surface. The term "planning" rectum surface refers to the rectum surfaces derived from rectum structures on planning CT, while "reference" rectum surface refers to the average rectum surface determined from rectum surfaces at all fractions. The algorithm to construct a reference surface was described in a paper by Shibayama et al. (Shibayama et al. 2017). On the other hand, the random error of a patient was defined as the SDs of the daily deviations 
of the rectum surface at each fraction from the reference rectum surface (ICRU 2010; Wu et al. 2001; Witte et al. 2004; Arimura et al. 2017).

\subsubsection{Systematic error}

Figure 3.3(a) illustrates the point distribution image of all the vertices on the reference rectum. To obtain the SDs of systematic and random errors due to shape variations along each direction, the rectum was split into two halves (anterior and posterior regions, left and right regions, superior and inferior regions) with respect to its centroid, depending on which direction was evaluated. The example is shown in Fig. 3.3(b), where the anterior half vertices were used to calculate the SDs of the systematic and random errors along the anterior direction.

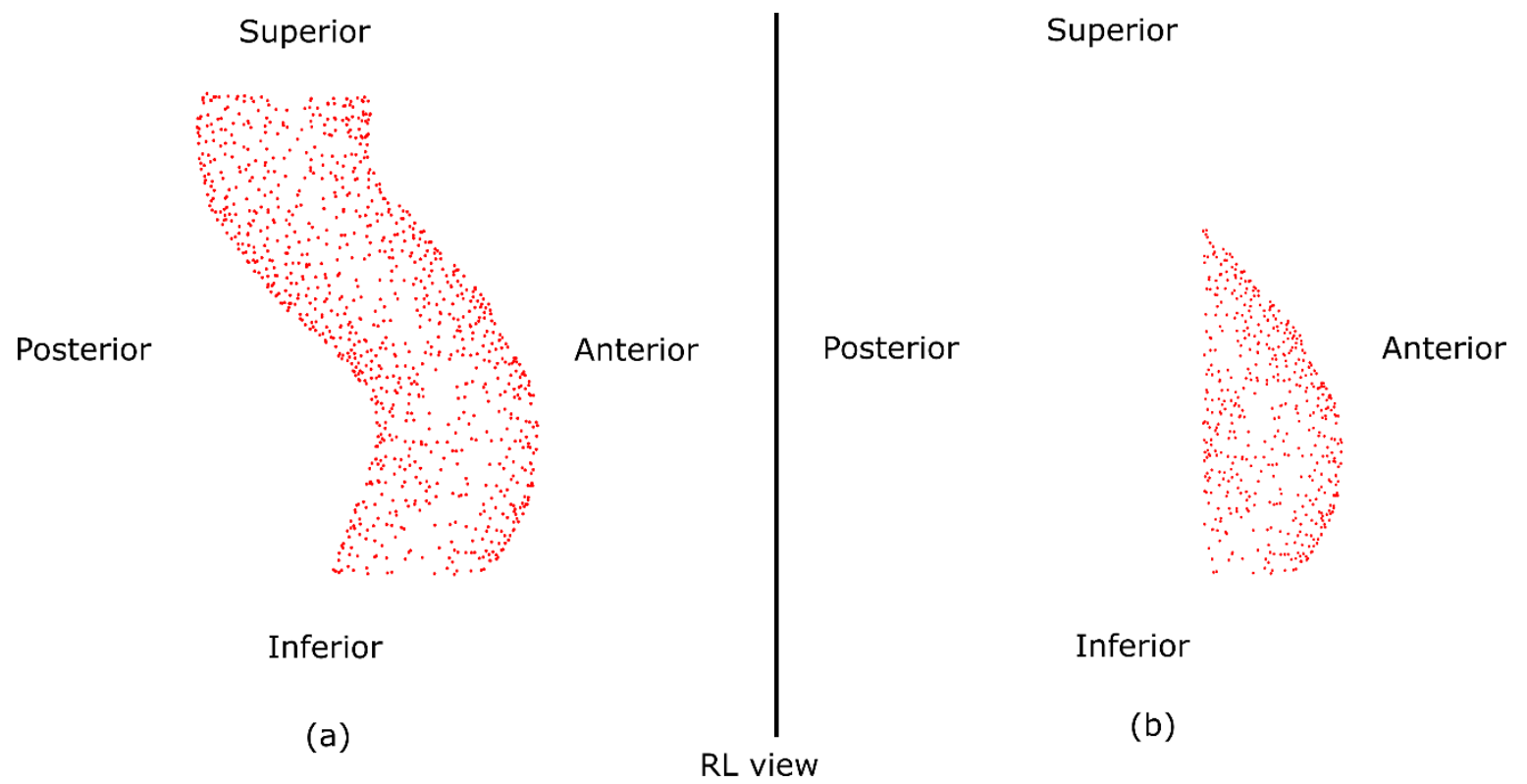

Fig. 3.3 Point distribution images consisting of reference rectum vertices which are used to evaluate shape variations viewed from RL direction: (a) the PDM of whole vertices while, (b) only the anterior part of the rectum vertices split at the centroid remains to be used in evaluating the shape variations along the anterior direction.

Figure 3.4 illustrates the determinations of the local systematic and random errors due to the shape variations. Let $\boldsymbol{R}_{i, r e f}$ be the reference rectum of the $i$-th patient $(i=1$, $2, \ldots, N ; N$ : number of patients ) which corresponded with the rectum at each fraction. Accordingly, the vector $\boldsymbol{p}_{i, j}(k)$ of the $k$-th vertex $(k=1,2, \ldots, M ; M$ : number of vertices) of the rectum at the $j$-th 
fraction $(j=1,2, \ldots, F ; F$ : number of fractions) of the $i$-th patient is corresponded to the vector of the $k$-th vertex $\left(\boldsymbol{p}_{i, r e f}(k)=\left(p_{i, r e f, x}(k), p_{i, r e f, y}(k), p_{i, r e f, z}(k)\right)^{\mathrm{T}}\right)$ at the reference rectum.

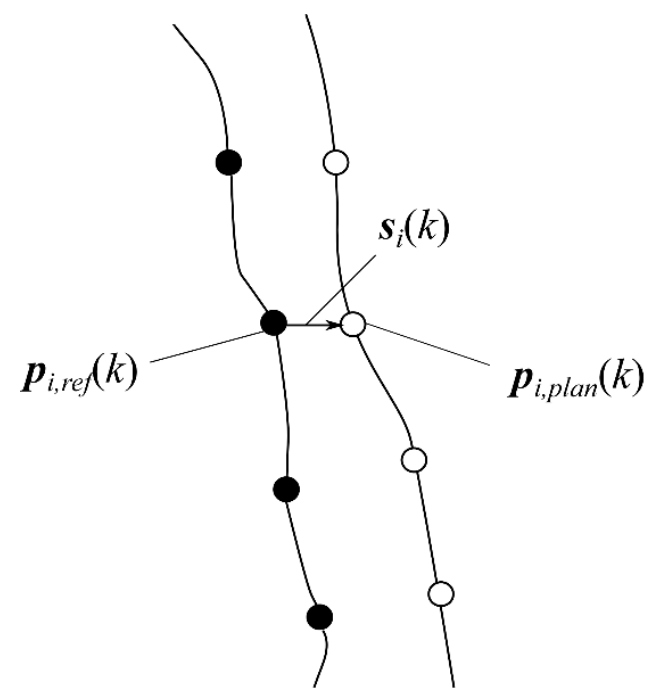

(a)

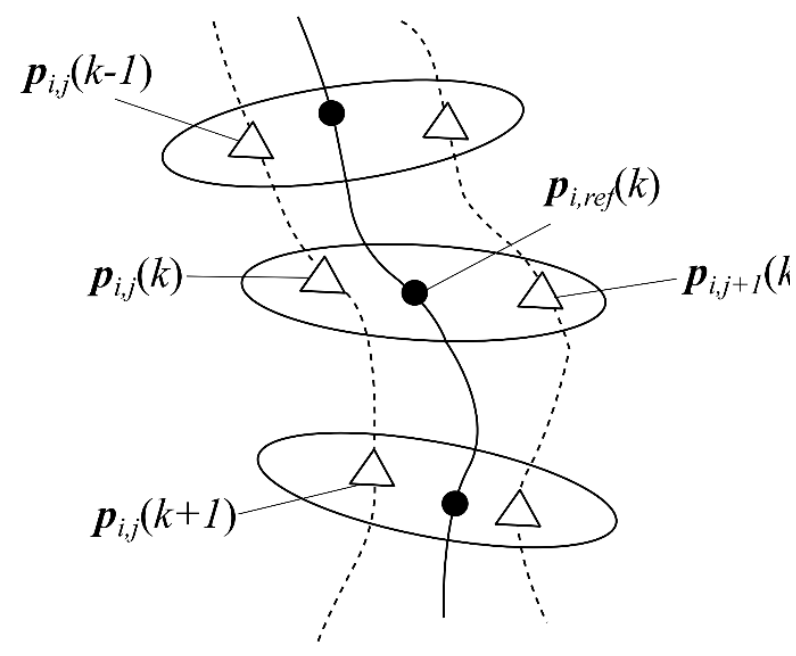

(b)

Fig. 3.4 Illustrations of the determinations of local systematic error and local random error due to shape variations: (a) local systematic error was calculated between reference rectum $(\bullet)$ and planning rectum ( $($ ) while, (b) local random error was calculated between reference rectum and rectum at each fraction $(\triangle)$.

The correspondence between the 3D vector at the $k$-th vertex $\left(\boldsymbol{p}_{i, p l a n}(k)=\left(p_{i, p l a n, x}(k)\right.\right.$, $\left.\left.p_{i, p l a n, y}(k), p_{i, p l a n, z}(k)\right)^{\mathrm{T}}\right)$ on the planning rectum $\boldsymbol{R}_{i, p l a n}$ and the vector of the $k$-th vertex $\boldsymbol{p}_{i, r e f}(k)$ on the reference rectum $\boldsymbol{R}_{i, r e f}$ was determined in a similar way. The local systematic error $\boldsymbol{s}_{i}(k)$ can be calculated as:

$$
\left.\boldsymbol{s}_{i}(k)=\boldsymbol{p}_{i, r e f}(k)-\boldsymbol{p}_{i, p l a n}(k)=\left(s_{i, x}(k), s_{i, y}(k), s_{i, z}(k)\right)^{\mathrm{T}}\right),
$$

where $s_{i, x}(k), s_{i, y}(k)$ and $s_{i, z}(k)$ represent the $x, y$ and $z$ components of the local systematic error vector in the inquired half, respectively. The average deviation $\varepsilon_{\mathrm{Sv}, i, A}$ for a patient along the anterior 
direction, in this study, can be calculated as (Stroom and Heijmen 2002; Arimura et al. 2017; Strbac and Jokic 2013; Dzierma et al. 2015):

$$
\varepsilon_{\mathrm{SV}, i, A}=\frac{1}{M_{A}} \sum_{k=1}^{M_{A}} s_{i, y}(k) .
$$

Here, $M_{A}$ denotes the number of vertices in the anterior half of the rectum surfaces. Then, the population SDs for all patients along the anterior direction can be obtained by (Stroom and Heijmen 2002; Arimura et al. 2017; Strbac and Jokic 2013; Dzierma et al. 2015):

$$
\varepsilon_{\mathrm{SV}, A}=\sqrt{\frac{1}{N} \sum_{1=1}^{N}\left(\varepsilon_{\mathrm{SV}, i, A}-\bar{\varepsilon}_{\mathrm{SV}, A}\right)^{2}}
$$

where

$$
\bar{\varepsilon}_{\mathrm{SV}, A}=\frac{1}{N} \sum_{1=1}^{N} \varepsilon_{\mathrm{SV}, i, A} .
$$

The population SD of the systematic error along the other directions were also calculated in a same way. The SDs of the local systematic error $\tau_{i}(k)$ at each vertex were calculated as:

$$
\tau_{i}(k)=\sqrt{s_{i, x}^{2}(k)+s_{i, y}^{2}(k)+s_{i, z}^{2}(k)} .
$$

\subsubsection{Random error}

The SDs of the interfractional random error due to shape variations were calculated by using a PDMbased covariance matrix. Let a point distribution column vector $\boldsymbol{q}_{i, j}$ of rectum $\boldsymbol{R}_{i, j}$ given by the elements of $\boldsymbol{p}_{i, j}(k)$ be: 


$$
\boldsymbol{q}_{i, j}=\left(p_{i, j, x}(1), \ldots, p_{i, j, x}(M), p_{i, j, y}(1), \ldots, p_{i, j, y}(M), p_{i, j, z}(1), \ldots, p_{i, j, z}(M)\right)^{\mathrm{T}}
$$

The covariance matrix at all fractions of the $i$-th patient can be obtained by calculating:

$$
\boldsymbol{V}_{i}=\frac{1}{F} \sum_{j=1}^{F}\left(\boldsymbol{q}_{i, j}-\overline{\boldsymbol{q}}_{i}\right)\left(\boldsymbol{q}_{i, j}-\overline{\boldsymbol{q}}_{i}\right)^{\mathrm{T}}
$$

where

$$
\overline{\boldsymbol{q}}_{i}=\frac{1}{F} \sum_{j=1}^{F} \boldsymbol{q}_{i, j}
$$

Here, the term $\overline{\boldsymbol{q}}_{i}$ denotes the mean vector of $\boldsymbol{q}_{i, j}$. The covariance matrix $\boldsymbol{V}_{i}$ consisted of diagonal elements which represent the square of SDs (variance) of the local random error at each vertex of the rectum. These elements can be defined as:

$$
\boldsymbol{v}_{\mathrm{SV}, i}=\left(\sigma_{\mathrm{SV}, i, x}^{2}(1), \ldots, \sigma_{\mathrm{SV}, i, x}^{2}(M), \sigma_{\mathrm{SV}, i, y}^{2}(1), \ldots, \sigma_{\mathrm{SV}, i, y}^{2}(M), \sigma_{\mathrm{SV}, i, z}^{2}(1), \ldots, \sigma_{\mathrm{SV}, i, z}^{2}(M)\right)^{\mathrm{T}}
$$

The variances on all vertices of the rectum were used to calculate the individual SD of the random error along each direction. The individual SD of a patient along the anterior direction $\left(\sigma_{\mathrm{SV}, i, A}\right)$ can be calculated as:

$$
\sigma_{\mathrm{SV}, i, A}=\sqrt{\frac{1}{M_{A}} \sum_{k=1}^{M_{A}} \sigma_{\mathrm{SV}, i, y}^{2}(k)},
$$

where $\sigma_{\mathrm{SV}, i, y}(k)$ denotes the local SD of the random error in the anterior half of the rectum and $M_{A}$ represents the number of vertices in the anterior half. The population SD of random error for all patients along the anterior direction $\left(\sigma_{\mathrm{SV}, A}\right)$ is defined as the root-mean-square (RMS) of the individual SDs of each patient in the group and calculated by (Stroom and Heijmen 2002; Strbac and Jokic 2013; Dzierma et al. 2015): 


$$
\sigma_{\mathrm{SV}, A}=\sqrt{\frac{1}{N} \sum_{i=1}^{N} \sigma_{\mathrm{SV}, i, A}^{2}}
$$

The population SDs along the other directions (left, right, posterior, superior and inferior) can be obtained using the same method. The SDs of the local random error on the $k$-th vertex $\left(\sigma_{\mathrm{Sv}, i}(k)\right)$ were calculated as:

$$
\sigma_{\mathrm{SV}, i}(k)=\sqrt{\sigma_{\mathrm{SV}, i, x}^{2}(k)+\sigma_{\mathrm{SV}, i, y}^{2}(k)+\sigma_{\mathrm{SV}, i, z}^{2}(k)} .
$$

\subsection{Calculations of errors at ROP regions}

The regions in which the rectum overlapped with the PTV along the anterior wall (referred to as ROP regions) may be included in high dose distributions which could lead to rectum toxicities such as rectal bleeding (Prabhakar et al. 2016). Therefore, the shape variations of the ROP regions were analyzed to improve the determination of the anisotropic PRV margins. The vertices of the rectum which were detected to be inside the PTV were selected. Then, the calculation of errors was conducted using similar principles described in Section 3.3.

\subsubsection{Determination of ROP regions}

The overall procedure for determining the ROP regions of the rectum is described in Fig. 3.5. The determination of correspondence between rectum at each fraction and a reference rectum will produce the corresponding rectum $\widehat{\boldsymbol{R}}_{i, j}$, where $j$ represents the fraction number $(j=$ $1,2, \ldots, F ; F$ : number of fractions $)$ of the $i$-th patient $(i=1,2, \ldots, N ; N$ : number of patients $)$. A reference PTV $\boldsymbol{T}_{i}$ of each patient was constructed by selecting the overlapped pixels between each PTV binary images at all fractions. 


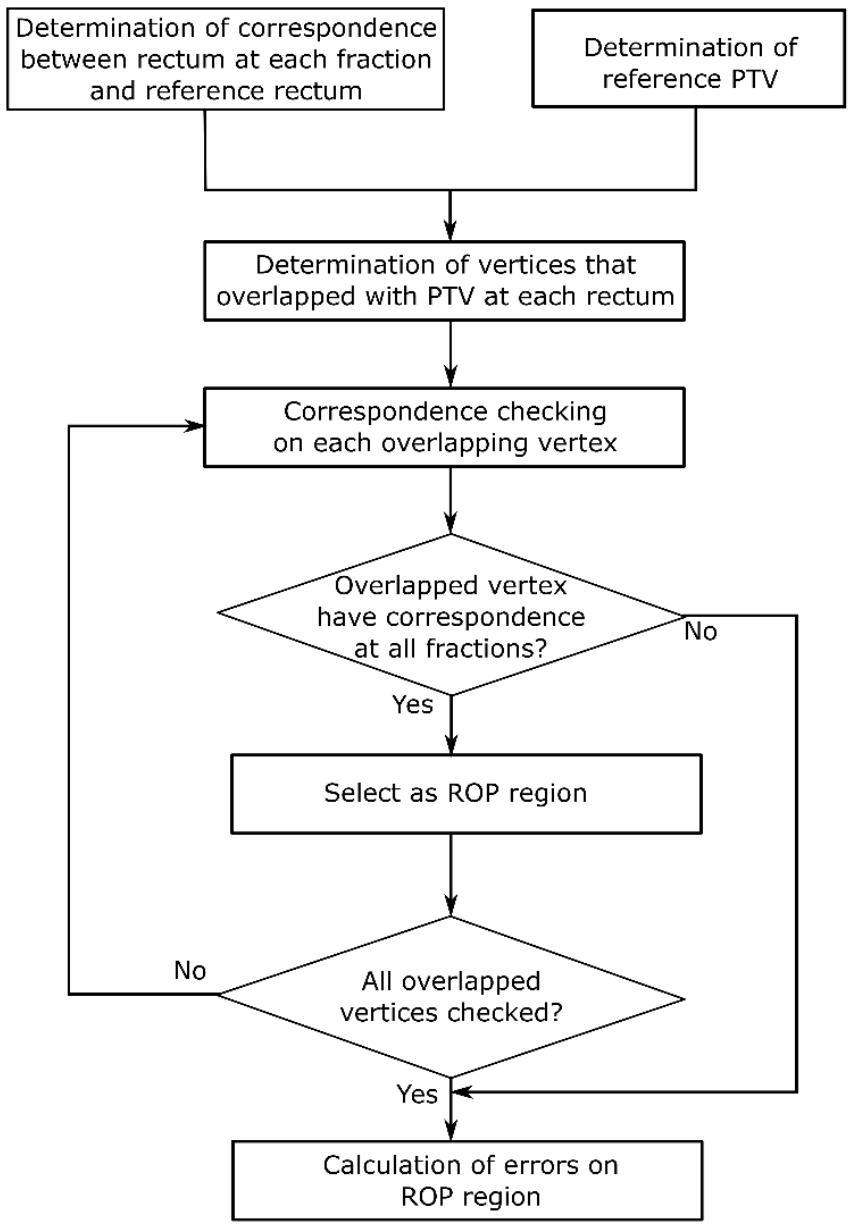

Fig. 3.5 A flowchart to calculate systematic and random errors of rectum-overlapped-with-PTV (ROP) regions due to shape variations using a statistical point distribution models (PDMs).

The overlapped vertices of the rectum $\widehat{\boldsymbol{R}}_{\mathrm{OV}, i, j}$ at the $j$-th fraction of the $i$-th patient were identified by finding the vertices whose coordinates were identical with those of the reference PTV $\boldsymbol{T}_{i}$. The process can be intuitively described as:

$$
\widehat{\boldsymbol{R}}_{\mathrm{OV}, i, j}=\widehat{\boldsymbol{R}}_{i, j} \cap \boldsymbol{T}_{i}
$$

Then, an ROP region at each fraction of a patient can be obtained by selecting overlapped vertices that have correspondences at all fractions as illustrated in Fig. 3.6. 


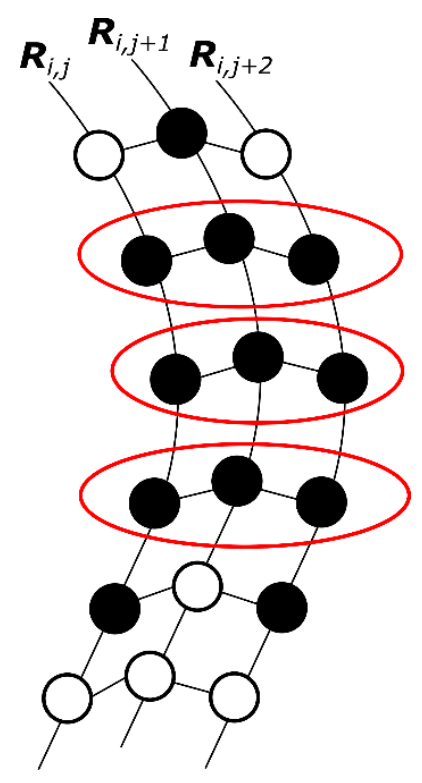

Fig. 3.6 An illustration of the determination of the ROP regions of the rectum. An overlapped vertex $(\bullet)$ would be included as ROP region (red border) if the correspondences with overlapped vertices at all fractions were found.

\subsection{Dose evaluation study}

In order to demonstrate the impacts of the proposed method on prostate cancer treatment, the treatment plans with an anisotropic PRV margin were designed as simulations on the CBCT images to calculate the dose evaluation indices, clinical target volume (CTV) $D_{98}$ and $V_{75}, V_{70}, V_{65}, V_{60}$ and $V_{40}$ of the rectum. $D_{98}$ was defined as the percentage of dose received by $98 \%$ of the CTV, while $V_{x}$ was defined as the rectum volume receiving $x \mathrm{~Gy}$. These dose evaluation indices were selected since $V_{70}$ and $V_{75}$ for the rectum were associated with Grade 2 or higher complications (E. H. Huang et al. 2002), while in another reports $V_{65}$ for the rectum was also associated with late toxicity (Pederson et al. 2012; Chennupati et al. 2014; Someya et al. 2014) and $V_{40}-V_{60}$ for the rectum were found to be more relevant to the occurrence of severe late rectal bleeding (Michalski et al. 2010; Coates and El Naqa 2016).

Four new cases were employed as test cases for validation of the PRV margin determined based on the shape variations, which were obtained by the proposed method in this study. The anisotropic PRV margin was derived along each direction by using the McKenzie's margin recipe of $1.3 \varepsilon+0.5 \sigma$ to the rectum (McKenzie, Van Herk, and Mijnheer 2002). Here, $\varepsilon$ and $\sigma$ denote the population SDs of 
the systematic error (Eq. (3.3)) and random errors (Eq. (3.11)), respectively, which were calculated by the proposed method. The test cases were treated with IMRT with a total number of 21 fractions. A treatment plan based on the PRV margin was generated for each patient by applying plan constraints to the rectum PRV using a commercially available RTP system (Eclipse version 10.0, Varian Medical Systems Inc., Palo Alto, USA). The constraints were $V_{70}<5 \%, V_{65}<10 \%, V_{60}<20 \%$ and $V_{40}<$ $40 \%$, which are based on QUANTEC recommendations to have been used in clinical practice (Michalski et al. 2010; Buschmann et al. 2016). A Wilcoxon signed-rank test was employed to compute the statistical difference between dose evaluation indices in original and PRV-based plans.

\subsection{Results}

Figure 3.7 shows the population SDs of the systematic and random errors of the rectum due to shape variations along each anatomical direction of all patients. The population SDs for systematic errors were $0.6 \mathrm{~mm}$ along the left direction, $0.3 \mathrm{~mm}$ along the right direction, $1.0 \mathrm{~mm}$ along the anterior direction, $0.7 \mathrm{~mm}$ along the posterior direction, $2.1 \mathrm{~mm}$ along the inferior direction and $2.4 \mathrm{~mm}$ along the superior direction. The population SDs for random errors were $1.2 \mathrm{~mm}$ along the left direction, $1.2 \mathrm{~mm}$ along the right direction, $1.6 \mathrm{~mm}$ along the anterior direction, $1.6 \mathrm{~mm}$ along the posterior direction, $1.9 \mathrm{~mm}$ along the inferior direction and $1.7 \mathrm{~mm}$ along the superior direction. 

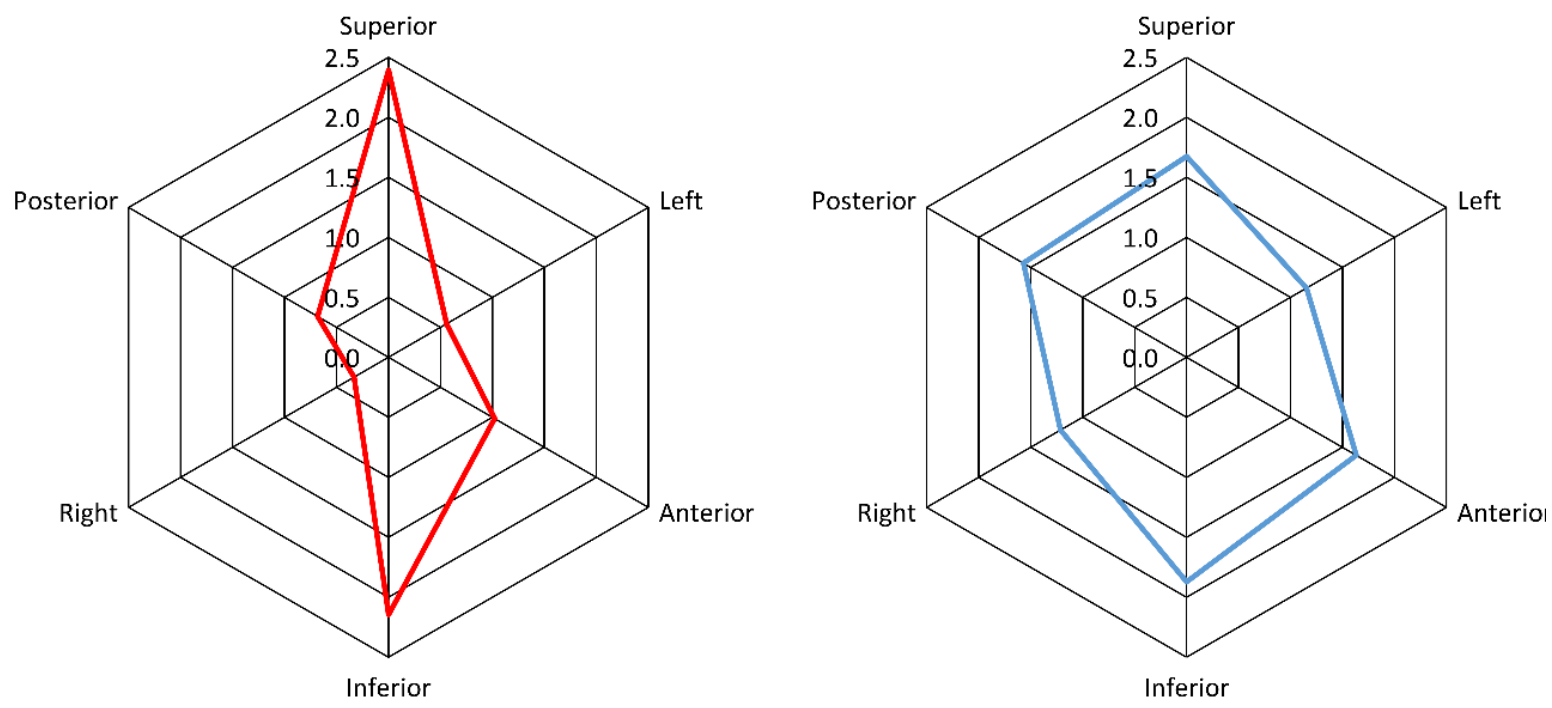

(a)

Systematic error

(b)

Random error

Fig. 3.7 The population SDs in mm of (a) systematic errors and (b) random errors of all patients due to shape variations along each anatomical direction.

Figure 3.8 shows the comparisons for the population SDs for shape variations between the whole rectum and the ROP regions along each direction. For systematic errors of the ROP regions, the population SDs were smaller along all directions except along the left and right directions compared with the population SDs of the whole rectum. For random errors of the ROP regions, only the population SDs along the left direction was not changed, while along the other directions the population SDs were smaller compared with population SDs of the whole rectum. The population SDs was shown along the posterior direction indicating that the ROP regions were not limited to the anterior side of the rectum. 


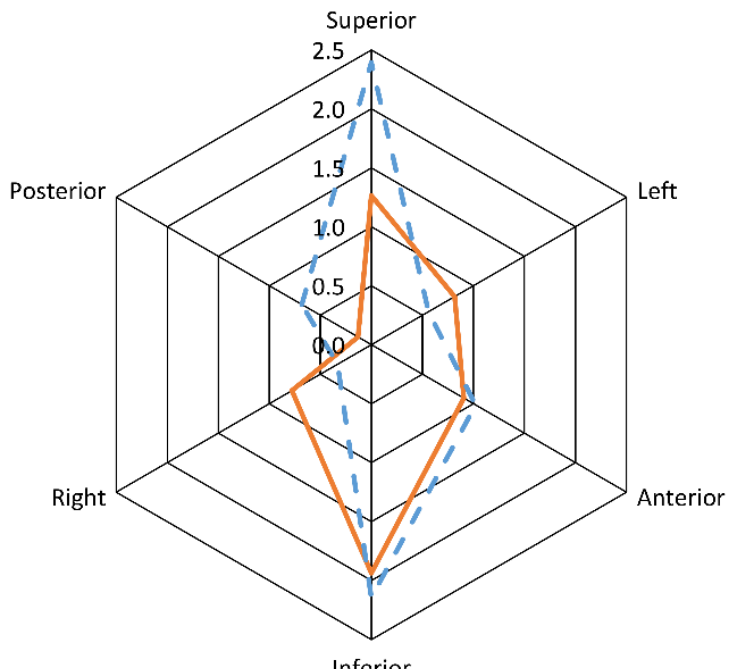

Inferior

(a)

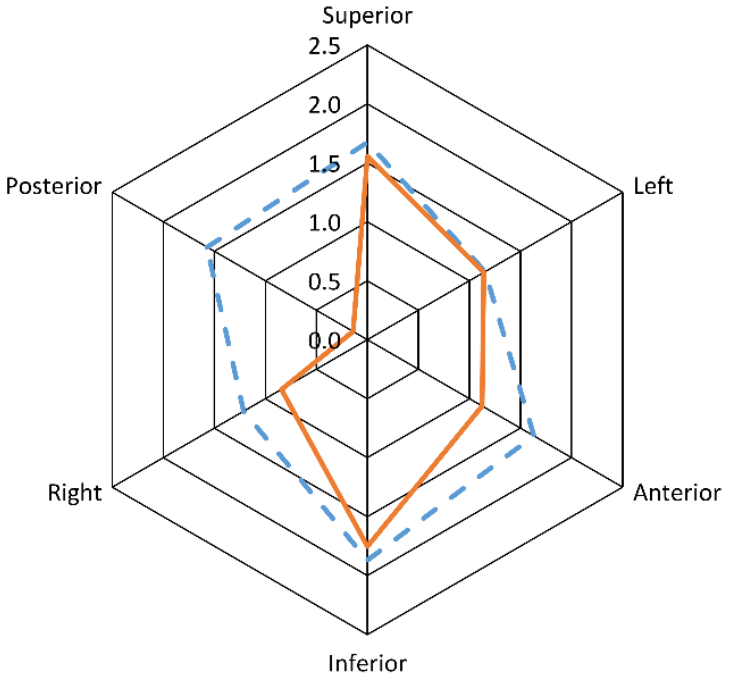

(b)

Fig. 3.8 The population SDs in mm of whole rectum and ROP regions of the rectum: (a) systematic error and (b) random errors of all patients due to shape variations along each direction.

The population SDs of systematic errors for the ROP regions were $0.8 \mathrm{~mm}$ along the left direction, $0.8 \mathrm{~mm}$ along the right direction, $0.9 \mathrm{~mm}$ along the anterior direction, $1.9 \mathrm{~mm}$ along the inferior direction and $1.3 \mathrm{~mm}$ along the superior direction. The population SDs of random errors for ROP regions were $1.1 \mathrm{~mm}$ along the left direction, $0.8 \mathrm{~mm}$ along the right direction, $1.1 \mathrm{~mm}$ along the anterior direction, $0.1 \mathrm{~mm}$ along the posterior direction, $1.8 \mathrm{~mm}$ along the inferior direction and $1.6 \mathrm{~mm}$ along the superior direction.

In summary, the population SDs of the random errors for the whole rectum along all directions were larger than $1.0 \mathrm{~mm}$. On the other hand, the population SDs of the systematic errors for the whole rectum were smaller than $1.0 \mathrm{~mm}$ along the posterior, left and right directions. The deviations along the superior direction was largest for the systematic errors, while the deviation along the inferior direction was largest for the random errors. The population SDs of systematic error for the ROP regions were larger than $1.0 \mathrm{~mm}$ along the superior and inferior directions, while the population SDs of random errors for the ROP regions were larger than $1.0 \mathrm{~mm}$ except along the right and posterior directions. 
Figure 3.9 shows the comparison of average dose evaluation indices between original and PRVbased plans for the four test patients. The $V_{75}$ for the rectum in PRV-based plans was significantly smaller $(\mathrm{p}<.001)$ than those of the original plans. However, the $V_{40}$ for the rectum in PRV-based plans were significantly larger $(\mathrm{p}<.001)$ than those of the original plans. On the other hand, the $D_{98}$ to the CTV showed no statistical significant difference between original and PRV-based plans ( $\mathrm{p}>$ $.001)$.

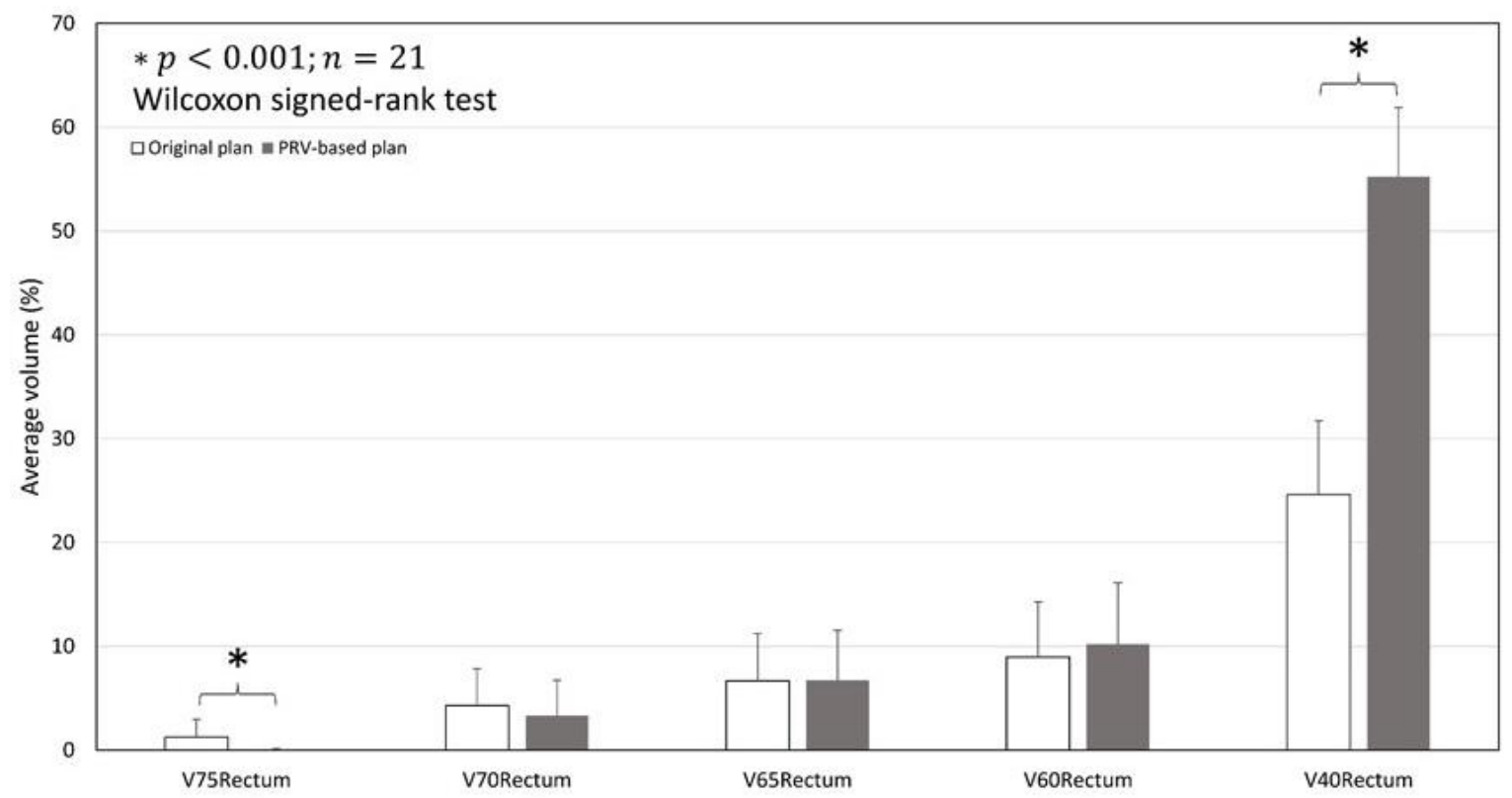

Fig. 3.9 Comparisons of average dose evaluation indices of the rectum (rectum $V_{75}, V_{70}, V_{65}, V_{60}$ and $V_{40}$ ) between original and PRV-based plans.

\subsection{Discussion}

The deviation along the superior, inferior and anterior directions were dominant for systematic and random errors. Brierley et al. and Nuyttens et al. noted similar observations of large deviations along superior and inferior directions of the rectum (Brierley et al. 2011; Nuyttens et al. 2002). The deviations were affected largely by the variabilities of other organ proximal to the rectum such as small bowel (Nuyttens et al. 2002). 
Figures 3.10 and 3.11 illustrate the SDs of the local systematic and random errors visualized on the reference rectum for a patient. The calculations of the magnitudes of the local systematic errors and local random errors were performed by Equations (3.5) and (3.12), respectively. Large variations (> $3.0 \mathrm{~mm}$ ) occurred in the upper-left and upper-anterior sides of the rectum for systematic errors. For random errors, beside the upper-left and upper-anterior side of the rectum, the lower-left and lower anterior side of the rectum also had variations larger than $3.0 \mathrm{~mm}$. Hoogeman et al. reported that their study also resulted in the largest variations occurred in the upper-anterior side of the rectum and uniformly distributed in the lower half of the rectum for random errors. The smaller variations in the posterior side of the rectum were indicating the possibilities of the sacrum limiting rectal wall movement (Hoogeman et al. 2004). The various SDs distributed on the rectum surface, as visualized in Fig. 3.10 and Fig. 3.11, showed that our method had more advantage in analysing rectum shape variations than volume-based methods since our method considered every voxel on the surface in the calculation.

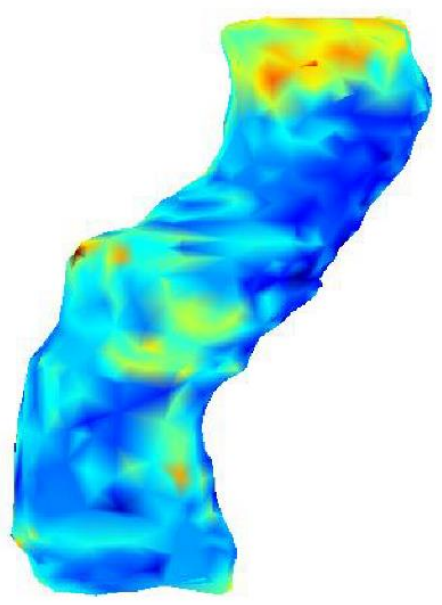

(a)

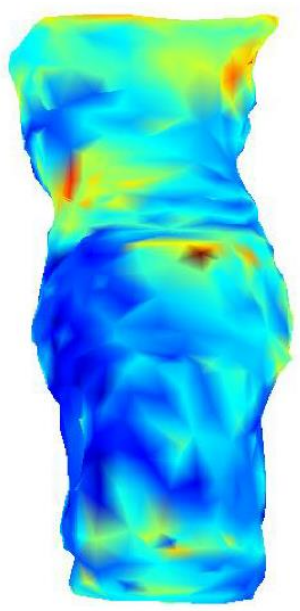

(b)

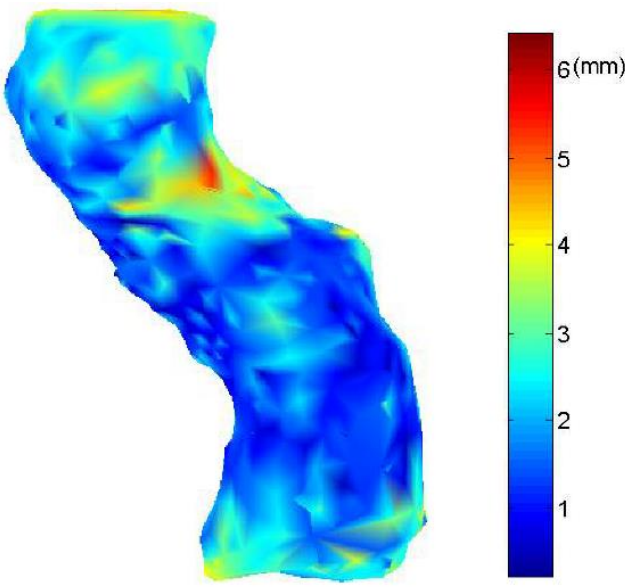

(c)

Fig. 3.10 Illustrations of the local systematic error SDs visualized on the reference rectum of a patient:

(a) LR view, (b) AP view and (c) RL view of rectum. 


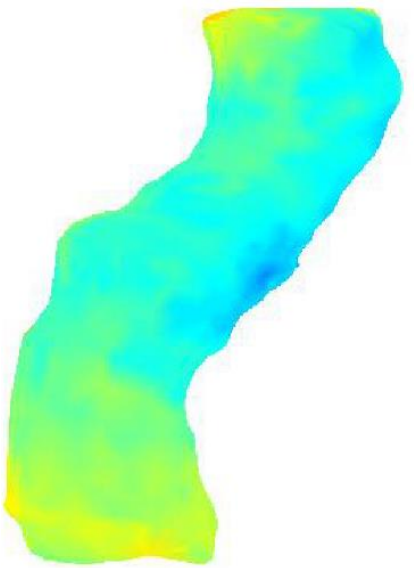

(a)

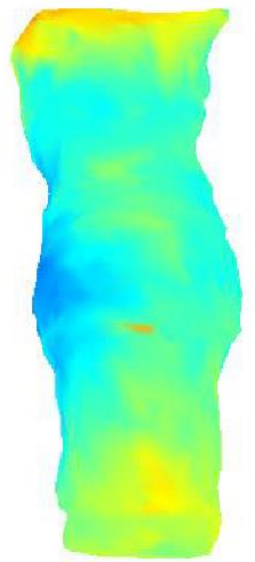

(b)

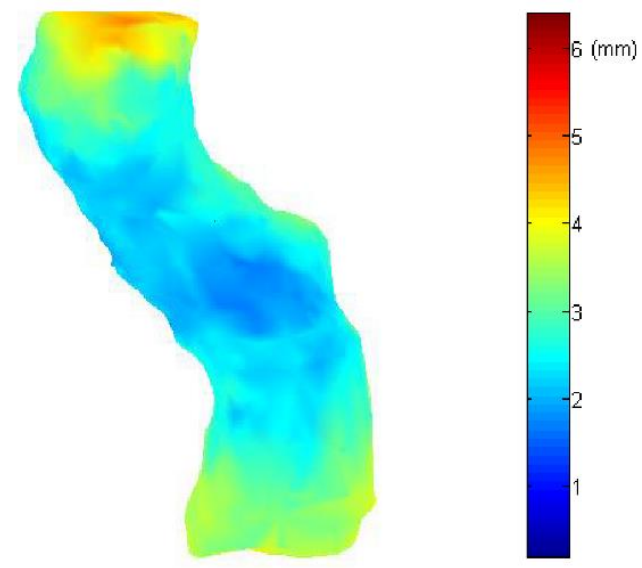

(c)

Fig. 3.11 Illustrations of the local random error SDs visualized on the reference rectum of a patient: (a) LR view, (b) AP view and (c) RL view of the rectum.

Figures 3.12 (a) and (b) illustrate the SDs of the local systematic and random errors of ROP region visualized on the reference rectum for a patient. Large variation $(>3.0 \mathrm{~mm})$ for systematic errors occurred in the upper part of the ROP regions. On the other hand, the variations $(>2.0 \mathrm{~mm})$ were uniformly distributed in the ROP regions for random errors. Aside from rectal gas and peristalsis activity (Shibayama et al. 2017), the lower part of the rectum was also suggested to be affected from the contraction and relaxation of the muscle system of the pelvic floor (Hoogeman et al. 2004). Variation larger than $3.0 \mathrm{~mm}$ in the ROP regions indicated that more portions of the rectum were exposed by the high dose region, consequently increasing the chance of acute toxicity in the rectum (Prabhakar et al. 2016). 


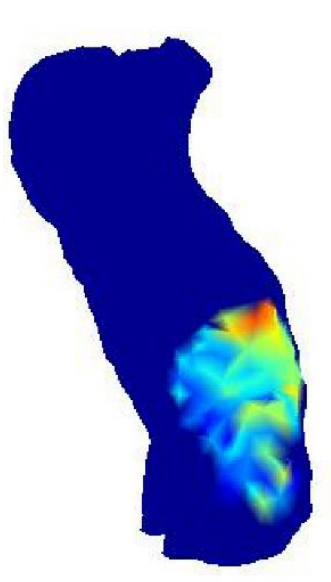

(a)

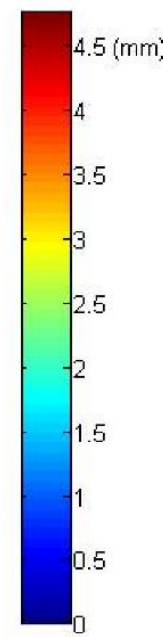

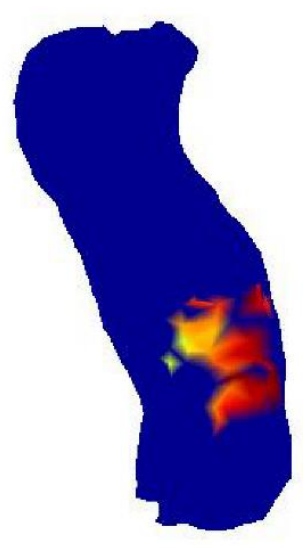

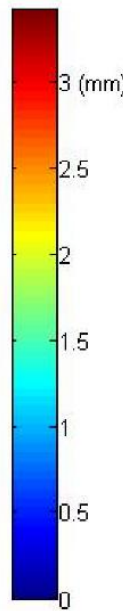

(b)

Fig. 3.12 Illustrations of the SDs of the local systematic and random errors of an ROP region visualized on the reference rectum of a patient: (a) local systematic and (b) local random error.

Figures 3.13 and 3.14 show the relationship between the SDs of dose evaluation indices and the individual SDs of systematic and random errors of rectum shape variations along the anterior direction, respectively. These results indicate that rectum shape variations affected variations of dose evaluation indices, but there were no statistical significances between them along all directions $(\mathrm{p}>$ .05 , Pearson correlation test). 

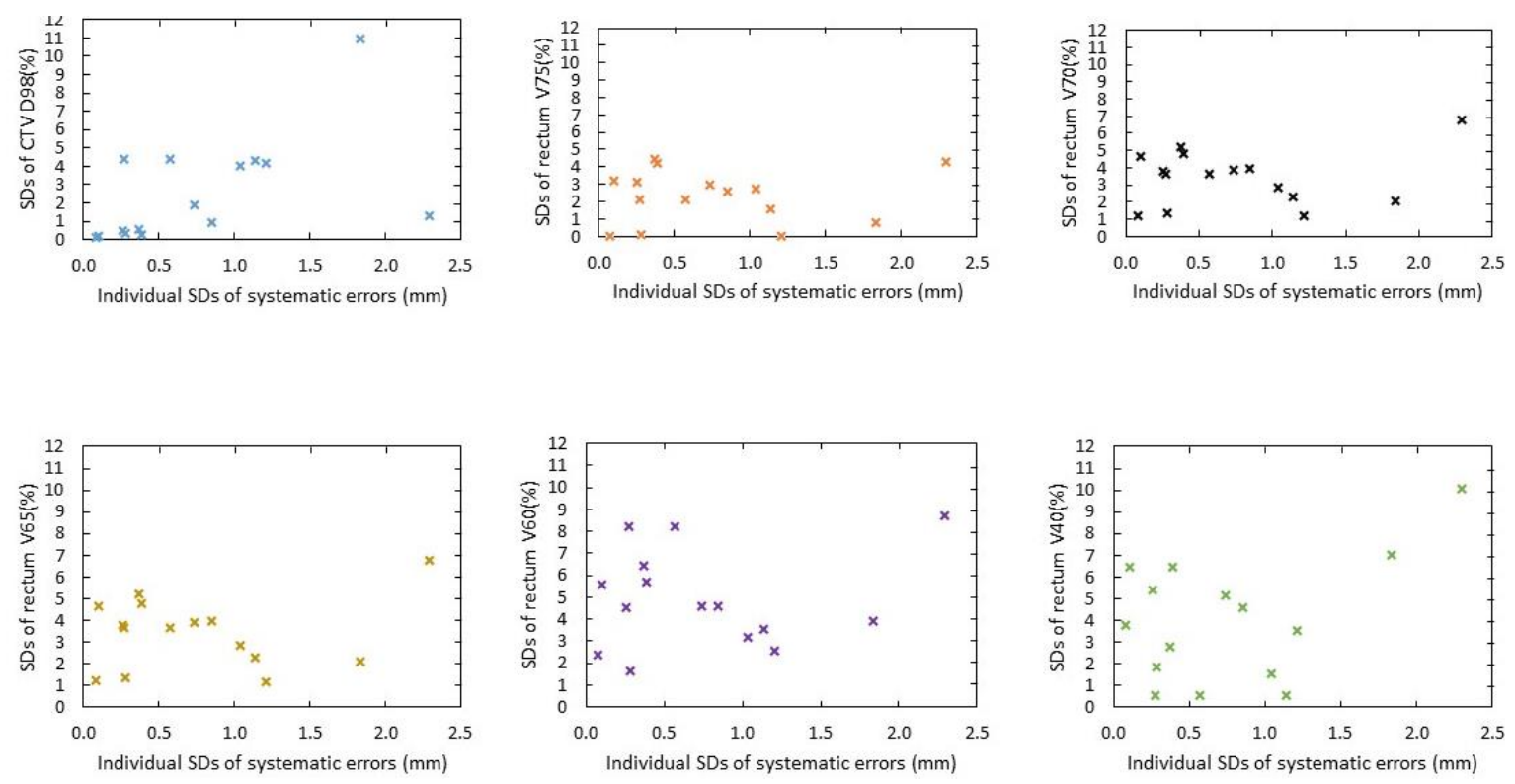

Fig. 3.13 Relationships between the SDs of dose evaluation indices and the individual SDs of systematic errors of rectum shape variations for 15 cases along the anterior direction.
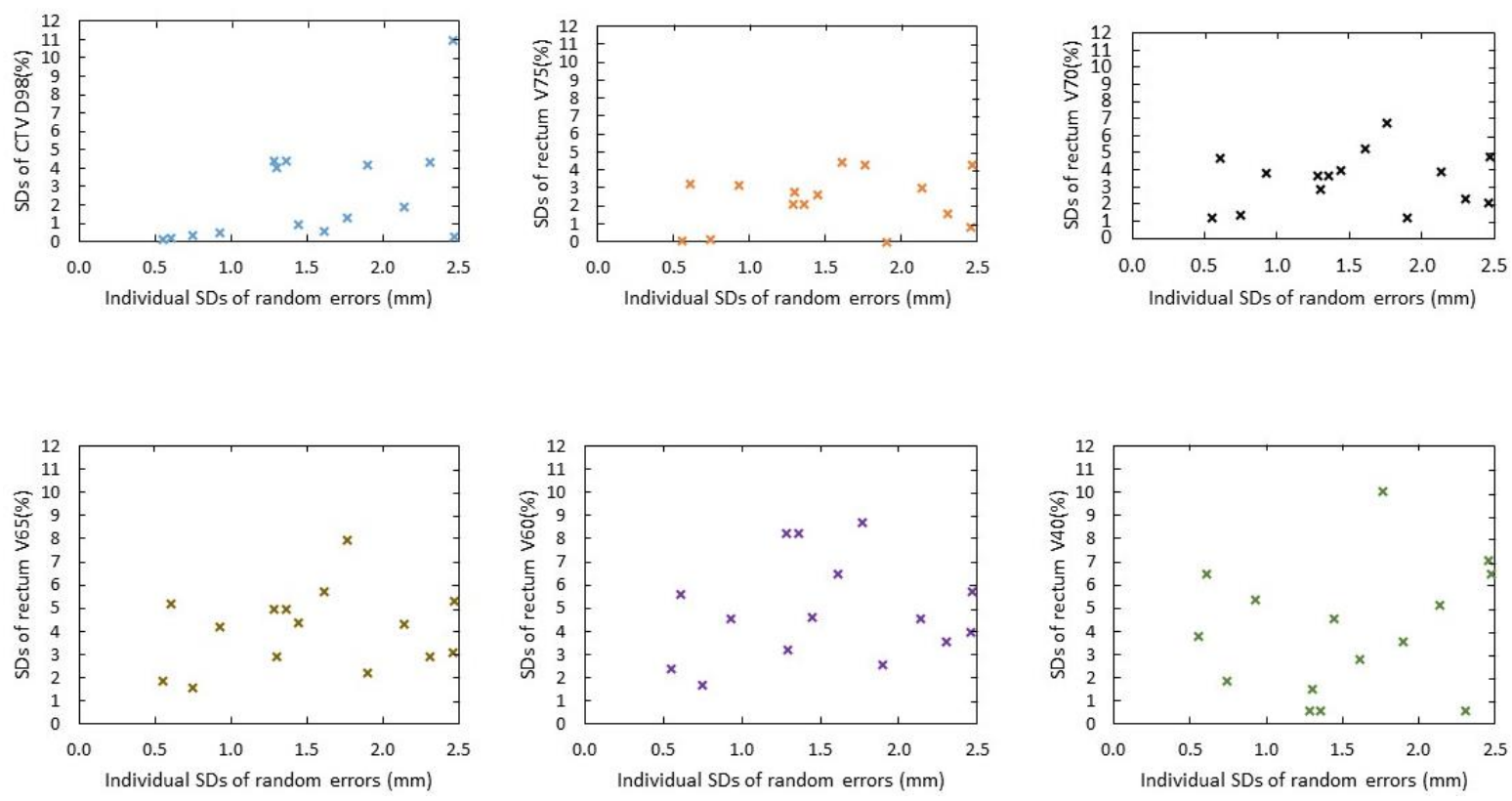

Fig. 3.14 Relationships between the SDs of dose evaluation indices and the individual SDs of random errors of rectum shape variations for 15 cases along the anterior direction.

The high dose to the ROP regions and large regions receiving low doses need to be studied in details to reduce rectum late toxicities and late gastrointestinal (GI) quality of life (QOL) in IMRT for prostate cancer in future studies. The ROP region is defined as the region in which the rectum overlapped with the PTV along the anterior wall and may be included in high dose distribution. 
Chennupati et al. reported that a high dose to the anterior rectal wall was associated with a low scoring of late GI QOL (Chennupati et al. 2014). On the other hand, as for large regions receiving low doses, dose constraints such as $V_{40}<65-80 \%$ were recommended to reduce the incidence rate of fecal incontinence to $1-2 \%$ (Landoni et al. 2016). Since $V_{40}$ obtained by the proposed method was smaller than $60 \%$, the risk of fecal incontinence may be negligible for the cases used in this study.

The smaller population SDs of the ROP regions than for the whole rectum may be used to improve the anisotropic PRV margins used in prostate cancer radiation treatment. A large margin for PRV of parallel organs such as the rectum could become problem if the PRV is used for dose evaluation and criteria determination (Stroom and Heijmen 2006). Since the rectal motion was reported to be occurring mainly along the anterior direction (Muren et al. 2004; Dias et al. 2011), the solution for providing a more compact margin can be obtained by deriving the PRV margins from the errors of the ROP regions especially along the anterior direction. By deriving the PRV margins based on the errors of the ROP regions, a smaller margin can be produced without neglecting the variations of the rectum wall proximal to the high dose regions. Smaller margins have also been indicated to be useful in predicting late morbidity of the rectum (Thor et al. 2010)

There are some limitations in this study. A larger number of cases is necessary to improve the statistical estimation of shape variations of the rectum. Since the TPS-RPM does not consider preserving the geometrical features of the surface, a more advanced correspondence determination method would also enhance the precision of the calculation of errors (Morooka and Nagahashi 2005; Miyauchi et al. 2013). Nevertheless, this study will be useful as a means to study the shape variations of the rectum. The results are envisioned as a means to derive anisotropic PRV margins for the rectum which can be utilized for general RTP systems. 


\section{Chapter 4 Conclusions}

In this thesis, we analysed the interfractional anisotropic rectum shape variations in prostate cancer radiation therapy. We proposed a computational framework to calculate the shape variation of the rectum and the ROP region by using a statistical PDM.

In Chapter 1, we introduced the potential of shape variation analysis of the rectum and introduced the ROP region of the rectum and the concept of PRV margin.

In Chapter 2, we introduced the basic theory related to the use of a statistical PDM to calculate the geometrical uncertainties in radiation therapy.

In Chapter 3, we proposed a computational framework for analysis of interfractional anisotropic shape variations of the rectum in prostate cancer radiation therapy. We evaluated the results by using a dose evaluation study to compare the original plan and the plan which used PRV margin derived from our proposed method. We concluded that that the anisotropic shape variations analysis, especially in the ROP regions, should be considered in the analysis of rectum uncertainties and the determination of PRV margins for the rectum associated with the acute toxicities. 


\section{References}

Arimura, Hidetaka, Yusuke Shibayama, Mohammad Haekal, Ze Jin, and Koujiro Ikushima. 2017. "Computer-Assisted Target Volume Determination." In Image-Based Computer-Assisted Radiation Therapy, edited by Hidetaka Arimura, 87-109. Singapore: Springer Singapore. doi:10.1007/978-981-10-2945-5_5.

Brierley, James D., Laura A. Dawson, Elliott Sampson, Andrew Bayley, Sandra Scott, Joanne L. Moseley, Timothy Craig, et al. 2011. "Rectal Motion in Patients Receiving Preoperative Radiotherapy for Carcinoma of the Rectum." International Journal of Radiation Oncology Biology Physics 80 (1): 97-102. doi:10.1016/j.ijrobp.2010.01.042.

Buschmann, Martin, Yvette Seppenwoolde, Tilo Wiezorek, Kirsten Weibert, and Dietmar Georg. 2016. "Advanced Optimization Methods for Whole Pelvic and Local Prostate External Beam Therapy." Physica Medica 32 (3): 465-73. doi:10.1016/j.ejmp.2016.03.002.

Chen, Rui, Shancheng Ren, Ming Kwong Yiu, Ng Chi Fai, Wai Sam Cheng, Lap Hong Ian, Seiji Naito, et al. 2014. "Prostate Cancer in Asia: A Collaborative Report." Asian Journal of Urology 1 (1): 15-29. doi:10.1016/j.ajur.2014.08.007.

Chennupati, Sravana K., Charles A. Pelizzari, Rangesh Kunnavakkam, and Stanley L. Liauw. 2014. "Late Toxicity and Quality of Life after Definitive Treatment of Prostate Cancer: Redefining Optimal Rectal Sparing Constraints for Intensity-Modulated Radiation Therapy." Cancer Medicine 3 (4): 954-61. doi:10.1002/cam4.261.

Chui, H., and A. Rangarajan. 2003. "A New Point Matching Algorithm for Non-Rigid Registration." Computer Vision and Image Understanding 89 (2-3): 114-41. doi:10.1016/S10773142(03)00009-2.

Coates, James, and Issam El Naqa. 2016. "Outcome Modeling Techniques for Prostate Cancer Radiotherapy: Data, Models, and Validation.” Physica Medica. doi:10.1016/j.ejmp.2016.02.014.

Cootes, T. F., and C. J. Taylor. 1992. "Active Shape Models - 'Smart Snakes."” In Procedings of the British Machine Vision Conference 1992, 28.1-28.10. doi:10.5244/C.6.28.

Cootes, Tf, and Cj Taylor. 2001. "Statistical Models of Appearance for Medical Image Analysis and Computer Vision.” Medical Imaging 2001, 236-48. doi:10.1117/12.431093.

Denmeade, Samuel R, and John T Isaacs. 2002. "A History of Prostate Cancer Treatment." Nat Rev Cancer 2 (5): 389-96. doi:10.1038/nrc801.

Dias, R S, A J Giordani, L Souhami, R A Segreto, and H R C Segreto. 2011. "Rectal Planning Risk 
Volume Correlation with Acute and Late Toxicity in 3-Dimensional Conformal Radiation Therapy for Prostate Cancer." Technology in Cancer Research \& Treatment 10 (6): 585-90.

Dzierma, Yvonne, Monica Beyhs, Jan Palm, Marcus Niewald, Katharina Bell, Frank Nuesken, Norbert Licht, and Christian R??be. 2015. "Set-up Errors and Planning Margins in Planar and CBCT Image-Guided Radiotherapy Using Three Different Imaging Systems: A Clinical Study for Prostate and Head-and-Neck Cancer." Physica Medica 31 (8): 1055-59. doi:10.1016/j.ejmp.2015.09.002.

Fontenla, E, C a Pelizzari, J C Roeske, and G T Chen. 2001. "Using Serial Imaging Data to Model Variabilities in Organ Position and Shape during Radiotherapy." Physics in Medicine and Biology 46 (9): 2317-36. doi:10.1088/0031-9155/46/9/304.

Foppiano, Franca, Claudio Fiorino, Giovanni Frezza, Carlo Greco, and Riccardo Valdagni. 2003. "The Impact of Contouring Uncertainty on Rectal 3D Dose-Volume Data: Results of a Dummy Run in a Multicenter Trial (AIROPROS01-02)." International Journal of Radiation Oncology Biology Physics 57 (2): 573-79. doi:10.1016/S0360-3016(03)00659-X.

Garland, Michael, and Paul S. Heckbert. 1997. "Surface Simplification Using Quadric Error Metrics." In Proceedings of the 24th Annual Conference on Computer Graphics and Interactive Techniques - SIGGRAPH '97, 209-16. doi:10.1145/258734.258849.

Haekal, Mohammad, Hidetaka Arimura, Taka aki Hirose, Yusuke Shibayama, Saiji Ohga, Junichi Fukunaga, Yoshiyuki Umezu, Hiroshi Honda, and Tomonari Sasaki. 2018. "Computational Analysis of Interfractional Anisotropic Shape Variations of the Rectum in Prostate Cancer Radiation Therapy." Physica Medica 46 (December 2017). Elsevier: 168-79. doi:10.1016/j.ejmp.2017.12.019.

Heimann, Tobias, and Hans Peter Meinzer. 2009. "Statistical Shape Models for 3D Medical Image Segmentation: A Review." Medical Image Analysis 13 (4): 543-63. doi:10.1016/j.media.2009.05.004.

Herman, Gabor T., Jingsheng Zheng, and Carolyn A. Bucholtz. 1992. "Shape-Based Interpolation." IEEE Computer Graphics and Applications 12 (3): 69-79. doi:10.1109/38.135915.

Hoogeman, Mischa S., Marcel Van Herk, Josien De Bois, Pietje Muller-Timmermans, Peter C.M. Koper, and Joos V. Lebesque. 2004. "Quantification of Local Rectal Wall Displacements by Virtual Rectum Unfolding.” Radiotherapy and Oncology 70 (1): 21-30. doi:10.1016/j.radonc.2003.11.015.

Hoogeman, Mischa S, Marcel van Herk, Di Yan, Liesbeth J Boersma, Peter C M Koper, and Joos V Lebesque. 2002. “A Model to Simulate Day-to-Day Variations in Rectum Shape.” Int J Radiat 
Oncol Biol Phys 54 (2): 615-25. doi:10.1016/S0360-3016(02)02946-2.

Huang, Eugene H., Alan Pollack, Larry Levy, George Starkschall, Lei Dong, Isaac Rosen, and Deborah A. Kuban. 2002. "Late Rectal Toxicity: Dose-Volume Effects of Conformal Radiotherapy for Prostate Cancer." International Journal of Radiation Oncology Biology Physics 54 (5): 1314-21. doi:10.1016/S0360-3016(02)03742-2.

Huang, Sandy H., Jia Pan, George Mulcaire, and Pieter Abbeel. 2015. "Leveraging Appearance Priors in Non-Rigid Registration, with Application to Manipulation of Deformable Objects." In IEEE International Conference on Intelligent Robots and Systems, 2015-Decem:878-85. doi:10.1109/IROS.2015.7353475.

ICRU. 2010. Prescribing, Recording and Reporting Photon-Beam Intensity Modulated Radiation Therapy (IMRT) (ICRU Report 83). Journal of the ICRU. Vol. 10. doi:10.1093/jicru/ndq025.

International Commission on Radiation Units and Measurements. 1999. "ICRU Report 62. Prescribing, Recording, and Reporting Photon Beam Therapy (Supplement to ICRU Report 50).” Journal of ICRU, no. November: Ix +52. doi:10.1259/bjr.74.879.740294.

Landoni, Valeria, Claudio Fiorino, Cesare Cozzarini, Giuseppe Sanguineti, Riccardo Valdagni, and Tiziana Rancati. 2016. "Predicting Toxicity in Radiotherapy for Prostate Cancer." Physica Medica 32 (3): 521-32. doi:10.1016/j.ejmp.2016.03.003.

Langen, K. M., and D. T L Jones. 2001. “Organ Motion and Its Management.” International Journal of Radiation Oncology Biology Physics. doi:10.1016/S0360-3016(01)01453-5.

Lebesque, Joos V., Allison M. Bruce, A. P. Guus Kroes, Adriaan Touw, Tarek Shouman, and Marcel van Herk. 1995. "Variation in Volumes, Dose-Volume Histograms, and Estimated Normal Tissue Complication Probabilities of Rectum and Bladder during Conformal Radiotherapy of T3 Prostate Cancer." International Journal of Radiation Oncology, Biology, Physics 33 (5): 1109-19. doi:10.1016/0360-3016(95)00253-7.

Long, Zhongjie, and Kouki Nagamune. 2015. "A Marching Cubes Algorithm: Application for ThreeDimensional Surface Reconstruction Based on Endoscope and Optical Fiber." International Information Institute (Tokyo). Information $18 \quad$ (4): $1425-37$. http://search.proquest.com/docview/1689384537/fulltext/523DA535F0EF4451PQ/1 ?accountid= 11107.

Lorensen, William E., and Harvey E. Cline. 1987. "Marching Cubes: A High Resolution 3D Surface Construction Algorithm.” COMPUTER GRAPHICS 21 (4): 163-169. doi:10.1145/37402.37422.

Mattes, Malcolm D., Jennifer C. Lee, Sara Elnaiem, Adel Guirguis, N. C. Ikoro, and Hani Ashamalla. 
2014. "A Predictive Model to Guide Management of the Overlap Region between Target Volume and Organs at Risk in Prostate Cancer Volumetric Modulated Arc Therapy.” Radiation Oncology Journal 32 (1): 23-30. doi:10.3857/roj.2014.32.1.23.

McKenzie, Alan, Marcel Van Herk, and Ben Mijnheer. 2002. "Margins for Geometric Uncertainty around Organs at Risk in Radiotherapy." Radiotherapy and Oncology 62 (3): 299-307. doi:10.1016/S0167-8140(02)00015-4.

Michalski, Jeff M., Hiram Gay, Andrew Jackson, Susan L. Tucker, and Joseph O. Deasy. 2010. "Radiation Dose-Volume Effects in Radiation-Induced Rectal Injury." International Journal of Radiation Oncology Biology Physics 76 (3 SUPPL.). doi:10.1016/j.ijrobp.2009.03.078.

Miyauchi, Shoko, Ken'ichi Morooka, Yasushi Miyagi, Takaichi Fukuda, Tokuo Tsuji, and Ryo Kurazume. 2013. "Tissue Surface Model Mapping onto Arbitrary Target Surface Based on SelfOrganizing Deformable Model.” In Emerging Security Technologies (EST), 2013 Fourth International Conference On., 79-82. Cambridge: IEEE. doi:10.1109/EST.2013.34.

Morooka, Ken'ichi, and Hiroshi Nagahashi. 2005. "Self-Organizing Deformable Model: A New Method for Fitting Mesh Model to given Object Surface.” In Lecture Notes in Computer Science (Including Subseries Lecture Notes in Artificial Intelligence and Lecture Notes in Bioinformatics), 3804 LNCS:151-58. doi:10.1007/11595755_19.

Muren, Ludvig Paul, Randi Ekerold, Yngve Kvinnsland, Àsa`Karlsdottir, and Olav Dahl. 2004. “On the Use of Margins for Geometrical Uncertainties around the Rectum in Radiotherapy Planning.” Radiotherapy and Oncology 70 (1): 11-19. doi:10.1016/j.radonc.2003.11.013.

Muren, Ludvig Paul, Ása Karlsdottir, Yngve Kvinnsland, Tore Wentzel-Larsen, and Olav Dahl. 2005. “Testing the New ICRU 62 'Planning Organ at Risk Volume' Concept for the Rectum." Radiotherapy and Oncology 75 (3): 293-302. doi:10.1016/j.radonc.2005.03.007.

Nuyttens, Joost J., John M. Robertson, Di Yan, and Alvaro Martinez. 2002. "The Variability of the Clinical Target Volume for Rectal Cancer due to Internal Organ Motion during Adjuvant Treatment.” International Journal of Radiation Oncology Biology Physics 53 (2): 497-503. doi:10.1016/S0360-3016(02)02753-0.

Pederson, Aaron W., Janine Fricano, David Correa, Charles A. Pelizzari, and Stanley L. Liauw. 2012. "Late Toxicity after Intensity-Modulated Radiation Therapy for Localized Prostate Cancer: An Exploration of Dose-Volume Histogram Parameters to Limit Genitourinary and Gastrointestinal Toxicity." International Journal of Radiation Oncology Biology Physics 82 (1): 235-41. doi:10.1016/j.ijrobp.2010.09.058.

Prabhakar, Ramachandran, Richard Oates, Jones Daryl, Joe Chang, Moshi Geso, and Jim Cramb. 2016. 
"Rectal Complication Probability from Composite Volumes Derived from Daily Cone Beam Computed Tomography in Prostate Cancer Radiotherapy." J Cancer Res Ther. 12 (1): 374-78. doi:10.4103/0973-1482.174529.

Prabhakar, Ramachandran, Richard Oates, Daryl Jones, Tomas Kron, Jim Cramb, Farshad Foroudi, Moshi Geso, and Suki Gill. 2014. "A Study on Planning Organ at Risk Volume for the Rectum Using Cone Beam Computed Tomography in the Treatment of Prostate Cancer." Medical Dosimetry 39 (1): 38-43. doi:10.1016/j.meddos.2013.09.003.

Rijkhorst, Erik Jan, Annemarie Lakeman, Jasper Nijkamp, Josien de Bois, Marcel van Herk, Joos V. Lebesque, and Jan Jakob Sonke. 2009. "Strategies for Online Organ Motion Correction for Intensity-Modulated Radiotherapy of Prostate Cancer: Prostate, Rectum, and Bladder Dose Effects.” International Journal of Radiation Oncology Biology Physics 75 (4): 1254-60. doi:10.1016/j.ijrobp.2009.04.034.

Roeske, J C, Jeffrey D Forman, Carmen F Mesina, Tony He, C A Pelizzari, E Fontenla, S Vijayakumar, and George T Y Chen. 1995. "Evaluation of Changes in the Size and Location of the Prostate, Seminal Vesicles, Bladder, and Rectum during a Course of External Beam Radiation Therapy." Int J Radiat Oncol Biol Phys 33 (5): 1321-29. doi:10.1016/0360-3016(95)00225-1.

Shibayama, Yusuke, Hidetaka Arimura, Taka-aki Hirose, Takahiro Nakamoto, Tomonari Sasaki, Saiji Ohga, Norimasa Matsushita, Yoshiyuki Umezu, Yasuhiko Nakamura, and Hiroshi Honda. 2017. "Investigation of Interfractional Shape Variations Based on Statistical Point Distribution Model for Prostate Cancer Radiation Therapy.” Medical Physics 44 (5): 1837-45. doi:10.1002/mp.12217.

Sohn, M, M Birkner, D Yan, and M Alber. 2005. "Modelling Individual Geometric Variation Based on Dominant Eigenmodes of Organ Deformation: Implementation and Evaluation." Physics in Medicine and Biology 50 (24): 5893-5908. doi:10.1088/0031-9155/50/24/009.

Someya, Masanori, Masakazu Hori, Kunihiko Tateoka, Kensei Nakata, Masaru Takagi, Masato Saito, Naoki Hirokawa, Masato Hareyama, and Koh Ichi Sakata. 2014. "Results and DVH Analysis of Late Rectal Bleeding in Patients Treated with 3D-CRT or IMRT for Localized Prostate Cancer." Journal of Radiation Research 56 (1): 122-27. doi:10.1093/jrr/rru080.

Strbac, Bojan, and Vesna Spasic Jokic. 2013. "Evaluation of Set-up Errors in Head and Neck Radiotherapy Using Electronic Portalimaging.” Physica Medica 29 (5): 531-36. doi:10.1016/j.ejmp.2012.12.001.

Stroom, Joep C., H. C J De Boer, Henk Huizenga, and Andries G. Visser. 1999. "Inclusion of Geometrical Uncertainties in Radiotherapy Treatment Planning by Means of Coverage Probability." International Journal of Radiation Oncology Biology Physics 43 (4): 905-19. 
doi:10.1016/S0360-3016(98)00468-4.

Stroom, Joep C., and Ben J.M. Heijmen. 2002. "Geometrical Uncertainties, Radiotherapy Planning Margins, and the ICRU-62 Report." Radiotherapy and Oncology 64 (1): 75-83. doi:10.1016/S0167-8140(02)00140-8.

2006. "Limitations of the Planning Organ at Risk Volume (PRV) Concept." International Journal of Radiation Oncology*Biology*Physics $66 \quad$ (1): 279-86. doi:10.1016/j.ijrobp.2006.05.009.

Thor, Maria, Michael Væth, Asa Karlsdottir, and Ludvig Paul Muren. 2010. "Rectum Motion and Morbidity Prediction: Improving Correlation between Late Morbidity and DVH Parameters through Use of Rectum Planning Organ at Risk Volumes.” Acta Oncologica 49 (7): 1061-68. doi:10.3109/0284186X.2010.505200.

Torre, L A, F Bray, R L Siegel, J Ferlay, J Lortet-Tieulent, and A Jemal. 2015. “Global Cancer Statistics, 2012." CA: A Cancer Journal for Clinicians 65 (2): 87-108. doi:10.3322/caac.21262 [doi].

van der Wielen, Gerard J., Theodore F. Mutanga, Luca Incrocci, Wim J. Kirkels, Eliana M. Vasquez Osorio, Mischa S. Hoogeman, B. J M Heijmen, and H. C J de Boer. 2008. "Deformation of Prostate and Seminal Vesicles Relative to Intraprostatic Fiducial Markers.” International Journal of Radiation Oncology Biology Physics 72 (5). doi:10.1016/j.ijrobp.2008.07.023.

Van Herk, Marcel. 2004. "Errors and Margins in Radiotherapy." Seminars in Radiation Oncology. doi:10.1053/j.semradonc.2003.10.003.

van Herk, Marcel, Allison Bruce, A. P. Guus Kroes, Tarek Shouman, Adriaan Touw, and Joos V. Lebesque. 1995. "Quantification of Organ Motion during Conformal Radiotherapy of the Prostate by Three Dimensional Image Registration." International Journal of Radiation Oncology, Biology, Physics 33 (5): 1311-20. doi:10.1016/0360-3016(95)00116-6.

Witte, Marnix G., Joris van der Geer, Christoph Schneider, Joos V. Lebesque, and Marcel van Herk. 2004. "The Effects of Target Size and Tissue Density on the Minimum Margin Required for Random Errors.” Medical Physics 31 (11): 3068-79. doi:10.1118/1.1809991.

Wu, Jackson, Tara Haycocks, Hamideh Alasti, Geri Ottewell, Nancy Middlemiss, Mohamed Abdolell, Padraig Warde, Ants Toi, and Charles Catton. 2001. "Positioning Errors and Prostate Motion during Conformal Prostate Radiotherapy Using on-Line Isocentre Set-up Verification and Implanted Prostate Markers." Radiotherapy and Oncology 61 (2): 127-33. doi:10.1016/S01678140(01)00452-2.

Zelefsky, M J, Z Fuks, L Happersett, H J Lee, C C Ling, C M Burman, M Hunt, et al. 2000. "Clinical 
Experience with Intensity Modulated Radiation Therapy (IMRT) in Prostate Cancer." Radiotherapy and Oncology: Journal of the European Society for Therapeutic Radiology and Oncology 55 (3): 241-49. doi:10.1016/S0167-8140(99)00100-0.

Zelefsky, Michael J., Diane Crean, Gig S. Mageras, Olga Lyass, Laura Happersett, C. Clifton Ling, Steven A. Leibel, et al. 1999. "Quantification and Predictors of Prostate Position Variability in 50 Patients Evaluated with Multiple CT Scans during Conformal Radiotherapy.” Radiotherapy and Oncology 50 (2): 225-34. doi:10.1016/S0167-8140(99)00011-0.

Zelefsky, Michael J., Zvi Fuks, Margie Hunt, Yoshiya Yamada, Christine Marion, C. Clifton Ling, Howard Amols, E. S. Venkatraman, and Steven A. Leibel. 2002. "High-Dose Intensity Modulated Radiation Therapy for Prostate Cancer: Early Toxicity and Biochemical Outcome in 772 Patients.” International Journal of Radiation Oncology Biology Physics 53 (5): 1111-16. doi:10.1016/S0360-3016(02)02857-2. 


\section{Achievements}

\section{Book Chapters and Articles}

1. Hidetaka Arimura, Yusuke Shibayama, Mohammad Haekal, et al., Computer-Assisted Target Volume Determination, In: Image-Based Computer-Assisted Radiation Therapy (Ed. Hidetaka Arimura, Springer), pp. 87-109, 03, 2017.

2. Hidetaka Arimura, Mazen Soufi, Mohammad Haekal, Multidimensional Image Analysis for High Precision Radiation Therapy, Japanese Journal of Medical Physics (医学物理), Vol. 36, No. 04, pp. 217-221, 2016.

\section{Peer-Reviewed Original Articles}

1. Mohammad Haekal, Hidetaka Arimura, Taka-aki Hirose, et al., Computational Analysis of Interfractional Anisotropic Shape Variations of the Rectum in Prostate Cancer Radiation Therapy, Physica Medica: European Journal of Medical Physics (EJMP), Vol. 46, pp. 168-179, 2018.

\section{Peer-Reviewed International Conference Proceedings}

1. Mohammad Haekal, Hidetaka Arimura, Yusuke Shibayama, et al., SU-I-GPD-T-237: An Investigation of Interfractional Rectum Shape Variations Based On a Statistical Shape Analysis for Prostate Cancer Radiotherapy, Medical Physics, Vol. 44, No. 6, 2017 (AAPM 59 2017, Colorado, Denver, USA).

\section{Peer-Reviewed Domestic Conference Proceedings}

1. Mohammad Haekal, Hidetaka Arimura, Yusuke Shibayama, et al., An Investigation of Geometric Uncertainties of the Rectum Surfaces due to the Shape Variations in Prostate Cancer Radiation Therapy, In Proceedings: 115th Conference of Japan Society for Medical Physics, 04, 2018 (JSMP115, Yokohama, Japan). 
2. Mohammad Haekal, Hidetaka Arimura, Yusuke Shibayama, et al., Computational Analysis of Rectum Translation Variability in Prostate Cancer Radiotherapy, In Proceedings: 113th Conference of Japan Society for Medical Physics, 04, 2017 (JSMP113, Yokohama, Japan).

3. Mohammad Haekal, Freddy Haryanto, Zamzam Ibnu Sina, Hidetaka Arimura, Algorithm Performance Evaluation: Gradient-descent, Simulated Annealing, and Hybrid Method for Finding an Optimum of a Function, In Proceedings: 111th Conference of Japan Society for Medical Physics, 04, 2016 (JSMP111, Yokohama, Japan).

\section{Domestic Conference Presentations}

1. Mohammad Haekal, Hidetaka Arimura, Yusuke Shibayama, et al., An Investigation of Geometric Uncertainties of the Rectum Surfaces due to the Shape Variations in Prostate Cancer Radiation Therapy, In Proceedings: 115th Conference of Japan Society for Medical Physics, 04, 2018 (JSMP115, Yokohama, Japan) (accepted).

2. Mohammad Haekal, Hidetaka Arimura, Yusuke Shibayama, et al., Computational Analysis of Rectum Translation Variability in Prostate Cancer Radiotherapy, In Proceedings: 113th Conference of Japan Society for Medical Physics, 04, 2017 (JSMP113, Yokohama, Japan).

3. Mohammad Haekal, Hidetaka Arimura, et al., Computational Analysis of Rectum Positional Variability by Centroid Measurement in Prostate Cancer Treatment, In Proceedings: The 117th Meeting of Medical Image Information (MII) Society, 01, 2017 (MII 2017, Fukuoka, Japan).

4. Mohammad Haekal, Freddy Haryanto, Zamzam Ibnu Sina, Hidetaka Arimura, Algorithm Performance Evaluation: Gradient-descent, Simulated Annealing, and Hybrid Method for Finding an Optimum of a Function, In Proceedings: 111th Conference of Japan Society for Medical Physics, 04, 2016 (JSMP111, Yokohama, Japan).

\section{International Conference Presentations}

1. Mohammad Haekal, Hidetaka Arimura, Yusuke Shibayama, et al., SU-I-GPD-T-237: An Investigation of Interfractional Rectum Shape Variations Based On a Statistical Shape Analysis for Prostate Cancer Radiotherapy, Medical Physics, Vol. 44, No. 6, 2017 (AAPM 59 2017, Colorado, Denver, USA) (Poster presentation). 
Fellowships and Scholarships:

October 2015 - September 2018: Japanese Government (Monbukagakusho) Scholarship 